\title{
The Odonata of Argentina: state of knowledge and updated checklist
}

\author{
F. Lozano (i)*, A. del Palacio, L. Ramos and J. Muzón \\ Laboratorio de Biodiversidad y Genética Ambiental (BioGeA), Universidad Nacional de Avellaneda, \\ Piñeyro, Argentina
}

(Received 7 February 2020; accepted 28 February 2020)

\begin{abstract}
An updated checklist of the 282 species of Odonata known to occur in Argentina is presented along with distributional information by province and ecoregion. Ten new records for the country and 87 new provincial records are provided. At present, 17 species of Odonata are considered endemic to Argentina, and distribution maps for each of them are provided. Information on larvae and conservation status according to the IUCN Red List of Threatened species is also provided; there are still 98 larvae unknown and 169 species unassessed.
\end{abstract}

Keywords: Zygoptera; Anisoptera; dragonfly; damselfly; new records

\section{Introduction}

The insect order Odonata includes conspicuous freshwater insects with hemimetabolous cycles that inhabit a wide variety of aquatic habitats including some water saturated terrestrial ones. Odonatology in Argentina began at the beginning of the twentieth century with collecting trips made by European museums (Calvert, 1909; Navás, 1917, 1920, 1927a, 1927b, 1928; Ris, 1904, $1913,1918,1928)$. These first contributions recorded approximately half of the current known diversity. However, advances on this group remained slow and sporadic; in 1947 and 1948 Fraser provided the first list of species for Argentina based on bibliography and material deposited in the collection of the Fundación Miguel Lillo (Tucumán). It was not until the 1970s, with the contributions of L.A. Bulla (1970, 1971, 1972, 1973a, 1973b, 1973-74), the first Argentinean Odonatologist, that the knowledge of Odonata started to develop steadily.

Traditionally, the main focus of odonatology in Argentina has been taxonomy; ecological papers on Odonata are very few (Campos, 1994; Muzón, Rodrigues Capitulo, \& Jurzitza, 1990; Ramos, Lozano, \& Muzón, 2017; Rodrigues Capitulo, 1981, 2000; Weigel Muñoz, Ramos, \& Muzón, 2019), so collections were intended to broaden the knowledge of alpha diversity of the country. In 1992, Rodrigues Capitulo published the first modern checklist, which included 224 species grouped in 11 families. Subsequently, in 1998, Muzón and von Ellenrieder provided a

*Corresponding author. Email: flozano@undav.edu.ar 

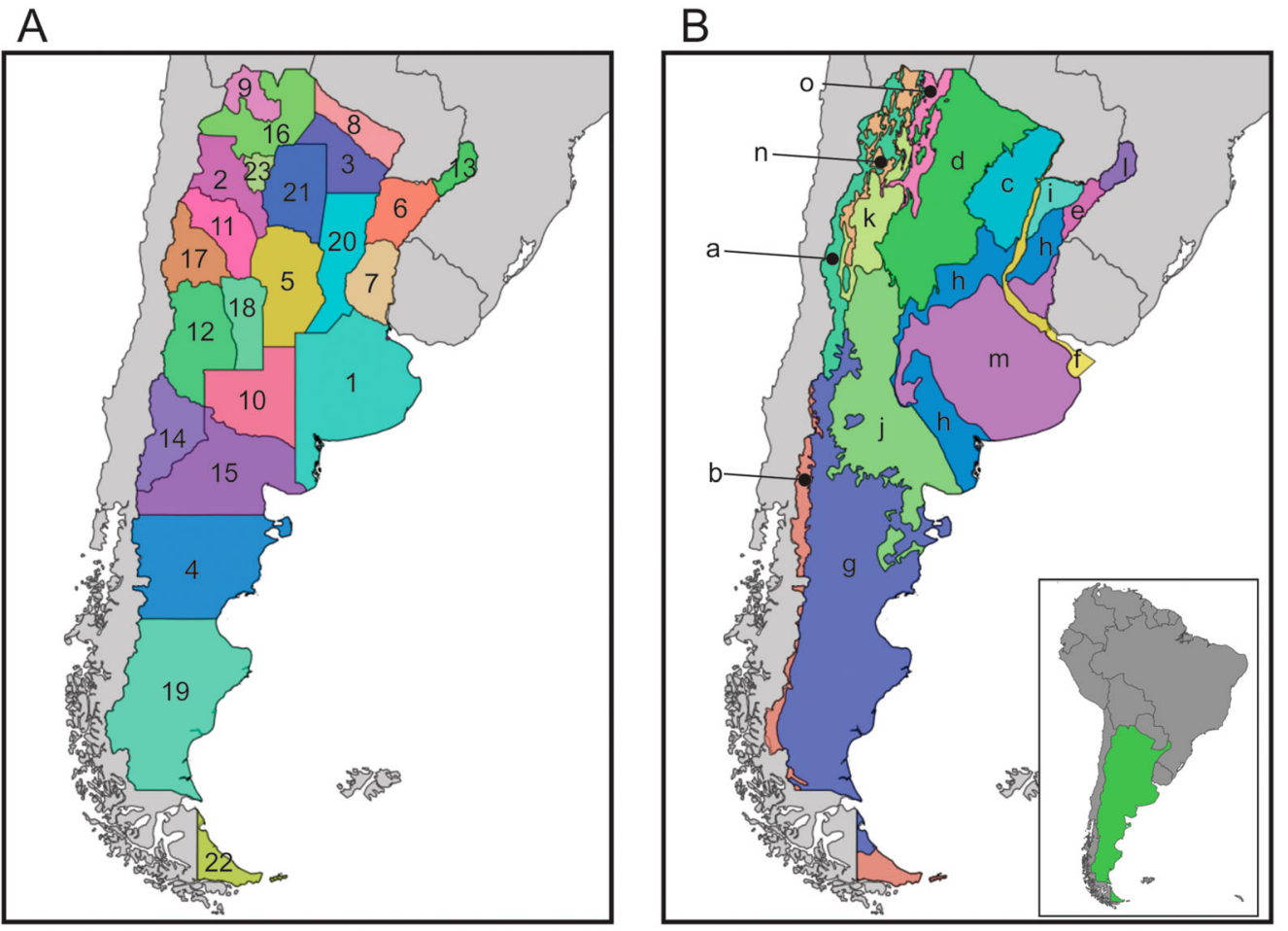

Figure 1. Maps. (A) Provinces: 1, Buenos Aires; 2, Catamarca; 3, Chaco; 4, Chubut; 5, Córdoba; 6, Corrientes; 7, Entre Ríos; 8, Formosa; 9, Jujuy; 10, La Pampa; 11, La Rioja; 12, Mendoza; 13, Misiones; 14, Neuquén; 15, Rio Negro; 16, Salta; 17, San Juan; 18, San Luis; 19, Santa Cruz; 20, Santa Fe; 21, Santiago del Estero; 22, Tierra del Fuego; 23, Tucumán. (B) Ecoregions: a, Altos Andes; b, Bosques Patagónicos; c, Chaco Húmedo; d, Chaco Seco; e, Campos y Malezales; f, Delta e Islas del Paraná; g, Estepa Patagónica; h, Espinal; i, Esteros del Iberá; j, Monte de Llanuras y Mesetas; k, Monte de Sierras y Bolsones; 1, Selva Paranaense; m, Pampa; n, Puna; o, Yungas.

new catalog clarifying the situation of many previous erroneous citations and registering for the first time for the country 37 species and four families. In 2008, von Ellenrieder and Muzón updated the specific inventory and provided a new list with 256 species within 15 families; they included 14 undescribed species.

Argentina is a vast country (mainland area of 2,780,400 $\mathrm{km}^{2}$ ) located in southern South America. It is subdivided into 23 provinces and one autonomous city, Buenos Aires, which is the federal capital of the nation (Figure 1A). Climatic conditions range from subtropical with hot weather in the north to subantarctic in the far south. Consequently, there is a wide variety of ecoregions within the country; according to Brown and Pacheco (2006), there are 18 ecoregions including the marine platform (Figure 1B). Its latitudinal extent, topography and complex geological history provide a wide variety of ecological conditions that determine a complex biota. There are two main faunistic components, a neotropical one in the north and center of the country between $34^{\circ}$ and $36^{\circ} \mathrm{S}$, where the southernmost limit of many widespread American genera is found (e.g. Acanthagrion, Argia, Hetaerina, Erythemis, Miathyria, Micrathyria, Perithemis, Tauriphila, and Tramea), and a subantarctic one in the south that includes many remarkable Patagonian endemics (e.g. family Neopetaliidae) (Muzón, Pessacq, \& Lozano, 2014).

In this contribution we provide a revision of the state of knowledge of the order Odonata in Argentina and include an updated checklist of the species recorded up to December 2018 . 


\section{Materials and methods}

The taxonomic treatment follows the latest classification schemes (Carle, Kjer, \& May, 2015; Dijkstra et al., 2013). It is important to mention that members of former families Protoneuridae and Pseudostigmatidae are now included within Coenagrionidae, and the genus Gomphomacromia, now considered Synthemistidae, was previously included within Corduliidae.

For each species the following information is provided:

- IUCN Category. Information was obtained from the Red List of Threatened Species. Abbreviations follow those provided by the IUCN and are included between [] after name of species; [NA] stands for "Not Assessed".

- New records for the country are indicated with an *.

- Provincial records. Acronyms used as follows: BUE: Buenos Aires; CAT: Catamarca; CHA: Chaco; CHU: Chubut; CBA: Córdoba; COR: Corrientes; ENT: Entre Ríos; FOR: Formosa; JUJ: Jujuy; LAP: La Pampa; LAR: La Rioja; MEN: Mendoza; MIS: Misiones; NEU: Neuquén; RIO: Rio Negro; SAL: Salta; SJU: San Juan; SLU: San Luis; SCR: Santa Cruz; SFE: Santa Fe; SGO: Santiago del Estero; TIE: Tierra del Fuego; TUC: Tucumán. When acronyms are in italics it indicates a provincial record in which there is no certainty on locality; underlined acronyms indicate that it is a record either published after the checklist of 2008 or published before but not included in it, references indicated between (); bold type acronyms are new provincial records, with locality information coded and added between () (see list of localities below).

- Ecoregions. The scheme follows the proposal by Brown and Pacheco (2006). Ecoregions with odonatological records are indicated between () after provincial records. Abbreviations as follows: ALT: Altos Andes; BPA: Bosques Patagónicos; CHH: Chaco Húmedo; CHS: Chaco Seco; CYM: Campos y Malezales; DIP: Delta e Islas del Paraná; EPA: Estepa Patagónica; ESP: Espinal; EST: Esteros del Iberá; MLM: Monte de Llanuras y Mesetas; MSB: Monte de Sierras y Bolsones; SEL: Selva Paranaense; PAM: Pampa; PUN: Puna; YUN: Yungas.

- Larval description (L). References for larval descriptions are indicated between \{\}

- Additional information is provided if necessary.

Unless stated otherwise the specimens are deposited in de Laboratorio de Biodiversidad y Genética Ambiental (BioGeA) Collection. Other collections/databases mentioned: CSCA (California State Collection of Arthropods, Sacramento, CA, USA), FML (Fundación Miguel Lillo, Tucuman, Argentina), Manzo Database (Ramiro Manzo Database), RWG (Rosser W. Garrison Personal Collection), USNM (National Museum of Natural History, Washington, DC, USA), NvE (Natalia von Ellenrieder Personal Collection).

List of localities for new provincial records mentioned in this contribution (Figure 2):

BUE 1: Del Medio stream over NR $9\left(-33.3301^{\circ} \mathrm{S},-60.3032^{\circ} \mathrm{W}\right), 20 \mathrm{~m}$ asl, col. Muzón, Lozano, del Palacio \& Ramos, 18 February 2015.

BUE 2: Pergamino stream $\left(-34.0455^{\circ} \mathrm{S},-60.1947^{\circ} \mathrm{W}\right), 32 \mathrm{~m}$ asl, col. Muzón, Lozano, del Palacio \& Ramos, 20 February 2015.

BUE 3: Unnamed stream over PR $41\left(-34.0712^{\circ} \mathrm{S},-59.5076^{\circ} \mathrm{W}\right), 17 \mathrm{~m}$ asl, col. Muzón, Lozano, del Palacio \& Ramos, 20 February 2015.

BUE 4: Avellaneda, CEAMSE, coast of Rio de La Plata $\left(-34.6787^{\circ} \mathrm{S},-58.2802^{\circ} \mathrm{W}\right), 6 \mathrm{~m}$ asl, col. Muzón, 3 February 2016.

BUE 5: Avellaneda, CEAMSE, quarry $\left(-34.6825^{\circ} \mathrm{S},-58.2845^{\circ} \mathrm{W}\right), 3 \mathrm{~m}$ asl

BUE 5a: col. Muzón, Lozano \& Ramos, 6 December 2012.

BUE 5b: col. Muzón, Lozano \& Ramos, 27 December 2012.

BUE 5c: col. Muzón, Lozano \& Ramos, 3 January 2013. 


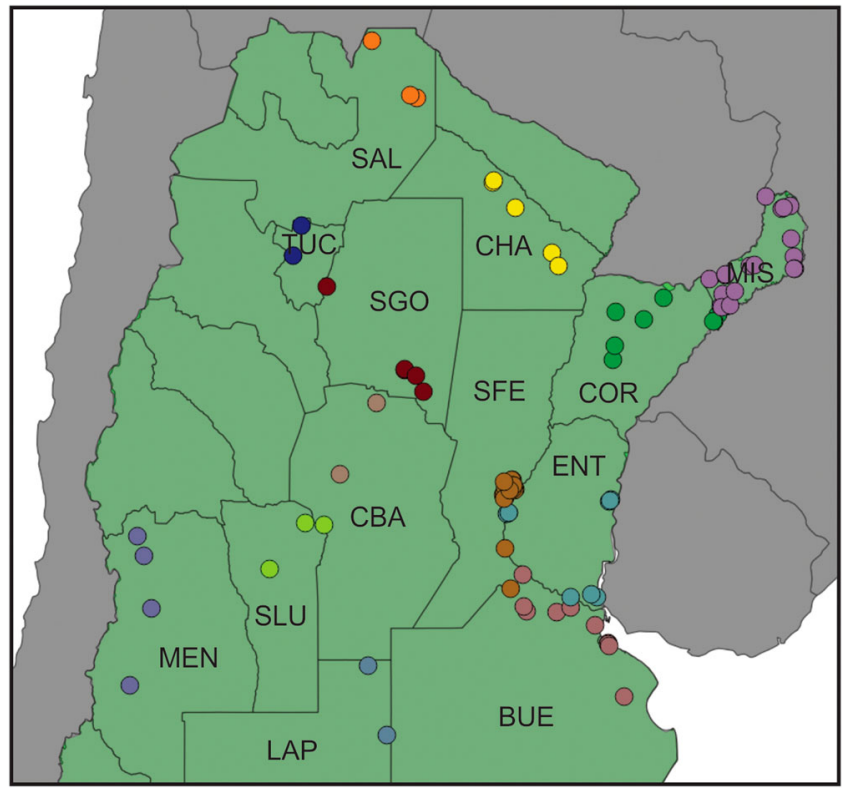

Figure 2. Map showing localities of new records.

BUE 5d: col. Muzón, Lozano \& Ramos, 17 January 2013. BUE 5e: col. Muzón, Lozano \& Ramos, 5 February 2013. BUE 5f: col. Muzón, Lozano \& Ramos, 29 December 2014. BUE 5g: col. Muzón, 3 February 2016.

BUE 6: Club de Pesca Lima, pond $\left(-33.9740^{\circ} \mathrm{S},-59.1773^{\circ} \mathrm{W}\right), 5 \mathrm{~m}$ asl, col. Muzón, 11 January 1995.

BUE 7: del Burro pond $\left(-35.6942^{\circ} \mathrm{S},-57.9219^{\circ} \mathrm{W}\right), 6 \mathrm{~m}$ asl, col. Bulla, November 1967.

BUE 8: Pergamino, Maguire stream and NR $8\left(-33.9665^{\circ} \mathrm{S},-60.2734^{\circ} \mathrm{W}\right), 47 \mathrm{~m}$ asl

BUE 8a: col. Muzón \& Pessacq, 15 January 2002.

BUE 8b: col. Muzón, Lozano, del Palacio \& Ramos, 19 February 2015.

BUE 9: Quilmes $\left(-34.7238^{\circ} \mathrm{S},-58.2628^{\circ} \mathrm{W}\right), 33 \mathrm{~m}$ asl, col. Lutz, 12 March 2015.

BUE 10: Tigre, Complejo Tabú Delta on Antequera river $\left(-34.3213^{\circ} \mathrm{S},-58.5850^{\circ} \mathrm{W}\right), 9 \mathrm{~m}$ asl, col. Muzón, Lozano, Ramos, del Palacio, Weigel Muñoz \& Lutz, 13-15 January 2014.

CHA 1: Pozo de la Gringa $\left(-25.3246^{\circ} \mathrm{S},-60.9891^{\circ} \mathrm{W}\right), 133 \mathrm{~m}$ asl, col. Lozano \& Lambruschini, 16-19 February 2008.

CHA 2: Bermejito river, $\left.-25.2842^{\circ} \mathrm{S},-60.9750^{\circ} \mathrm{W}\right), 132 \mathrm{~m}$ asl, col. Lambruschini \& Lozano, 18 February 2008.

CHA 3: Rio Negro $1 \mathrm{~km}$ of Chaco National Park $\left(-26.8106^{\circ} \mathrm{S},-59.5986^{\circ} \mathrm{W}\right), 81 \mathrm{~m}$ asl, col. Lozano \& Lambruschini, 23 February 2008.

CHA 4: Río Negro near Colonia Elisa and PR $9\left(-27.0766^{\circ} \mathrm{S},-59.4573^{\circ} \mathrm{W}\right), 67 \mathrm{~m}$ asl, col. Lozano \& Lambruschini, 23 February 2008.

CHA 5: Route $550 \mathrm{~km}$ from Puerto Lavalle $\left(-25.8633^{\circ} \mathrm{S},-60.4635^{\circ} \mathrm{W}\right), 110 \mathrm{~m}$ asl, col. Lozano \& Lambruschini, 20 February 2008.

CBA 1: Tanti $\left(-31.3541^{\circ} \mathrm{S},-64.5857^{\circ} \mathrm{W}\right), 996 \mathrm{~m}$ asl, col. Bulla \& Grosso, February 1968.

CBA 2: Villa María $\left(-29.9114^{\circ} \mathrm{S},-63.7256^{\circ} \mathrm{W}\right), 341 \mathrm{~m}$ asl, col. Flint Jr., 9 October 1973 , USNM

COR 1: Mburucuyá National Park, El Portillo stream on PR $86\left(-28.0364^{\circ} \mathrm{S},-58.1093^{\circ} \mathrm{W}\right)$, $67 \mathrm{~m}$ asl, col. Muzón, Lozano \& Ramos 
COR1a: 29 November 2010.

COR1b: 30 January 2012.

COR 2: Chimiray stream on PR $94\left(-28.0910^{\circ} \mathrm{S},-55.7066^{\circ} \mathrm{W}\right), 76 \mathrm{~m}$ asl, col. Muzón, Lozano \& Ramos, 2 February 2012.

COR 3: Pay Ubre Grande stream on PR $29\left(-29.0281^{\circ} \mathrm{S},-58.1744^{\circ} \mathrm{W}\right), 65 \mathrm{~m}$ asl

COR 3a: col. Muzón \& Pessacq, 21-23 February 2003.

COR 3b: col. Pessacq, 9-11 October 2004.

COR 3c: col. Muzón \& Lozano, 12-13 March 2010.

COR 3d: col. Muzón, Lozano \& Ramos, 26-27 November 2010.

COR 3e: col. Muzón, Lozano \& Ramos, 27 January 2012.

COR 3f: col. Muzón, Lozano, Ramos \& del Palacio, 27 January 2013.

COR 4: Pond on PR 22 (Tamborcito de Tacuarí) $\left(-27.7400^{\circ} \mathrm{S},-56.9941^{\circ} \mathrm{W}\right), 120 \mathrm{~m}$ asl, col. Muzón, Lozano \& Ramos, 31 January 2012.

COR 5: Estancia El Dorado, main house $\left(-28.7428^{\circ} \mathrm{S},-58.1267^{\circ} \mathrm{W}\right), 54 \mathrm{~m}$ asl, col. Pessacq \& Muzón, 20-21 February 2003.

COR 6: Estancia San Nicolás, Carambolas stream $\left(-28.1846^{\circ} \mathrm{S},-57.4429^{\circ} \mathrm{W}\right), 61 \mathrm{~m}$ asl

COR6a: col. Muzón \& Lozano, 16-17 March 2010.

COR6b: col. Muzón, Lozano \& Ramos, 27-28 November 2010.

COR 7: Garaví, pond on PR 94, south entrance of Garaví $\left(-28.2254^{\circ} \mathrm{S},-55.8000^{\circ} \mathrm{W}\right), 100 \mathrm{~m}$ asl, col. Muzón \& Lozano, 11 December 2009.

COR 8: Garaví, small ponds on PR $94\left(-28.2290^{\circ} \mathrm{S},-55.8096^{\circ} \mathrm{W}\right), 140 \mathrm{~m}$ asl

COR 8a: col. Muzón \& Lozano, 11 December 2009.

COR 8b: col. Muzón, Lozano \& Ramos, 2 February 2012.

ENT 1: Delta del Paraná, El Brasilero stream, Quinta Arco Iris $\left(-33.7841^{\circ} \mathrm{S},-58.5505^{\circ} \mathrm{W}\right), 9$ $\mathrm{m}$ asl

ENT 1a: col. Lutz, 25 November 2012.

ENT 1b: col. Pagano, 8 March 2014.

ENT 1c: col. Muzón, Ramos, del Palacio \& Lutz, 30-31 March 2014.

ENT 1d: col. Lutz, 8-10 February 2015.

ENT 1e: col. Jensen, 7 March 2015.

ENT 2: Ibicuy, Islas Malvinas camping site $\left(-33.7798^{\circ} \mathrm{S},-59.1721^{\circ} \mathrm{W}\right), 30 \mathrm{~m}$ asl, col. Muzón \& Lozano, 6-9 November 2006.

ENT 3: El Palmar National Park, Los Loros stream $\left(-31.8630^{\circ} \mathrm{S},-58.2292^{\circ} \mathrm{W}\right), 19 \mathrm{~m}$ asl, col. Rodrigues Capitulo, 23 November 1982.

ENT 4: El Palmar National Park, La Glorieta, Palmar stream $\left(-31.8874^{\circ} \mathrm{S},-58.2742^{\circ} \mathrm{W}\right), 25$ $\mathrm{m}$ asl

ENT 4a: col. Muzón, ix.1987.

ENT 4b: col. Muzón, Lozano, Ramos \& del Palacio, 29 January 2013.

ENT 5: El Palmar National Park, grassland $\left(-31.8818^{\circ} \mathrm{S},-58.2483^{\circ} \mathrm{W}\right), 40 \mathrm{~m}$ asl, col. Muzón, 20 September 1985.

ENT 6: Pre-Delta National Park, La Azotea stream (-32.1216 $\left.{ }^{\circ} \mathrm{S},-60.6332^{\circ} \mathrm{W}\right), 12 \mathrm{~m}$ asl, col. Lozano, Garré, Lambruschini, Ramos \& Weigel Muñoz, 23-25 November 2006.

ENT 7: Pre-Delta National Park, La Manga stream $\left(-32.1325^{\circ} \mathrm{S},-60.6756^{\circ} \mathrm{W}\right), 14 \mathrm{~m}$ asl, col. Lozano, Garré, Lambruschini, Ramos \& Weigel Muñoz, 24 November 2006.

ENT 8: Pre-Delta National Park, Park Ranger's house, pond $\left(-32.1216^{\circ} \mathrm{S},-60.6332^{\circ} \mathrm{W}\right), 12$ $\mathrm{m}$ asl, col. Lozano, del Palacio \& Manzo, 4 April 2014.

ENT 9: Villa Paranacito, surroundings of Top Malo camping site $\left(-33.7186^{\circ} \mathrm{S},-58.6808^{\circ} \mathrm{W}\right)$, 206 m asl, col. Muzón \& Lozano, 27-29 November 2018.

LAP 1: Catriló, $14.03 \mathrm{~km}$ of Catriló, NR $5\left(-36.4321^{\circ} \mathrm{S},-63.4958^{\circ} \mathrm{W}\right), 131 \mathrm{~m}$ asl, col. Latini, 27 October 2017. 
LAP 2: Chapaleufú, $9.94 \mathrm{~km}$ of Coronel Hilario Laos $\left(-35.1139^{\circ} \mathrm{S},-63.9314^{\circ} \mathrm{W}\right), 142 \mathrm{~m}$ asl, col. Latini, 24 January 2017.

MEN 1: Aguanda stream, Paso de las Carretas, Route $153\left(-34.0003^{\circ} \mathrm{S},-69.0208^{\circ} \mathrm{W}\right), 1180 \mathrm{~m}$ asl, col. Muzón \& Pessacq, 6 December 2005.

MEN 2: Malargüe $\left(-35.4759^{\circ} \mathrm{S},-69.5317^{\circ} \mathrm{W}\right), 1400 \mathrm{~m}$ asl, col. Muzón, Scheibler \& Ramos, 16 December 2007.

MEN 3: Potrerillos, ditch $\left(-32.9625^{\circ} \mathrm{S},-69.1845^{\circ} \mathrm{W}\right), 1372 \mathrm{~m}$ asl, col. Scheibler, April 2002.

MEN 4: Uspallata, Uspallata stream on NR $7\left(-32.5893^{\circ} \mathrm{S},-69.3512^{\circ} \mathrm{W}\right), 1830 \mathrm{~m}$ asl MEN 4a: col. Muzón, 27-29 January 2004.

MEN 4b: col. Muzón, 4 March 2005.

MIS 1: Apóstoles, PR 10, $\mathrm{km} \mathrm{13}$, pond $\left(-27.9154^{\circ} \mathrm{S},-55.6394^{\circ} \mathrm{W}\right), 140 \mathrm{~m}$ asl, col. Muzón \& Lozano, 10 December 2009.

MIS 2: Anyico stream on NR $14\left(-27.6645^{\circ} \mathrm{S},-55.6089^{\circ} \mathrm{W}\right), 150 \mathrm{~m}$ asl

MIS 2a: col. Muzón \& Lozano, 8 December 2009.

MIS 2b: col. Muzón \& Lozano, 15 November 2011.

MIS 2c: col. Muzón, Lozano \& Ramos, 4 February 2012.

MIS 3: León stream on PR $10\left(-27.9325^{\circ} \mathrm{S},-55.6178^{\circ} \mathrm{W}\right), 120 \mathrm{~m}$ asl, col. Muzón, Lozano \& Ramos, 4 February 2012.

MIS 4: Ponds on PR 2, km $34\left(-27.9041^{\circ} \mathrm{S},-55.4270^{\circ} \mathrm{W}\right), 180 \mathrm{~m}$ asl, col. Muzón \& Lozano, 9 December 2009.

MIS 5: Iguazú $\left(-25.6025^{\circ} \mathrm{S},-54.5692^{\circ} \mathrm{W}\right), 180 \mathrm{~m}$ asl, col. Tomsick \& Willink, 4 November 1973.

MIS 6: Leandro N. Alem $\left(-27.6066^{\circ} \mathrm{S},-55.3236^{\circ} \mathrm{W}\right), 315 \mathrm{~m}$ asl, col. Biraben, 29 November 1957.

MIS 7: Cruce Caballero Provincial Park, "Caraya Path" (-26.5056 ${ }^{\circ}$, $\left.-53.9983^{\circ} \mathrm{W}\right), 580 \mathrm{~m}$ asl, col. von Tschirnhaus, 12 March 2012.

MIS 8: Cuña Pirú Provincial Park, Cuña Pirú stream (-27.0864º S, $\left.-54.9537^{\circ} \mathrm{W}\right), 200 \mathrm{~m}$ asl, col. von Tschirnhaus, 20 March 2011.

MIS 9: Esmeralda Provincial Park, flooded area near Florida stream $\left(-26.8916^{\circ} \mathrm{S},-\right.$ 53.9138 $\left.{ }^{\circ} \mathrm{W}\right), 437 \mathrm{~m}$ asl, col. del Palacio, January 2016.

MIS 10: Salto Encantado Provincial Park, Salto la Olla $\left(-27.0627^{\circ} \mathrm{S},-54.8411^{\circ} \mathrm{W}\right), 325 \mathrm{~m}$ asl, col. von Tschirnhaus, 2011.

MIS 11: Saltos del Moconá Provincial Park, Yabotí stream $\left(-27.1456^{\circ} \mathrm{S},-53.9075^{\circ} \mathrm{W}\right), 299 \mathrm{~m}$ asl, col. von Tschirnhaus, 11 February 2011.

MIS 12: Saltos del Moconá Provincial Park, Oveja Negra stream, Salto Horacio $\left(-27.1410^{\circ}\right.$ S, $\left.-53.9239^{\circ} \mathrm{W}\right), 189 \mathrm{~m}$ asl, col. Muzón, Lozano \& Samways, 17 November 2011.

MIS 13: Urugua-í Provincial Park, $24 \mathrm{~km} \mathrm{SW}$ ' of Andresito $\left(-25.8570^{\circ} \mathrm{S},-54.1631^{\circ} \mathrm{W}\right), 280$ m asl, col. von Tschirnhaus, J., 2011.

MIS 14: Urugua-í Provincial Park, Destacamento de Gardaparques 101, $15 \mathrm{~km} \mathrm{~S}$ of Andresito, pond $\left(-25.8109^{\circ} \mathrm{S},-54.0136^{\circ} \mathrm{W}\right), 355 \mathrm{~m}$ asl

MIS 14a: col. von Tschirnhaus, 25 February 2011.

MIS 14b: col. von Tschirnhaus, 9-10 March 2011.

MIS 15: Urugua-í Provincial Park, Destacamento de Gardaparques Uruzú, $30 \mathrm{~km}$ SE of Andresito, Yatebo stream $\left(-25.8833^{\circ} \mathrm{S},-54.2167^{\circ} \mathrm{W}\right), 270 \mathrm{~m}$ asl, col. von Tschirnhaus, 5-6 March 2011.

MIS 16: Urugua-í Provincial Park, Destacamento de Gardaparques Uruzú, $23 \mathrm{~km}$ SW of Andresito, Playita stream $\left(-25.8572^{\circ} \mathrm{S},-54.1677^{\circ} \mathrm{W}\right), 280 \mathrm{~m}$ asl, col. von Tschirnhaus, 21-23 February 2011.

MIS 17: Posadas, Itá Provincial Park (Urquiza and Tomás Guido) $\left(-27.3592^{\circ} \mathrm{S},-55.9106^{\circ} \mathrm{W}\right)$, $89 \mathrm{~m}$ asl, col. Tejeda Cejas, 9 December 2010. 
MIS 18: San Ignacio, Loreto, Yabebiry stream $\left(-27.2832^{\circ} \mathrm{S},-55.5331^{\circ} \mathrm{W}\right), 103 \mathrm{~m}$ asl MIS 18a: col. Tejeda Cejas, 12 May 2010.

MIS 18b: col. von Tschirnhaus, 25 January 2011.

MIS 19: San Ignacio $\left(-27.2583^{\circ} \mathrm{S},-55.5392^{\circ} \mathrm{W}\right), 162 \mathrm{~m}$ asl, col. Biraben, August 1946.

SAL 1: NR 81 ponds with Pistia $\left(-23.5203^{\circ} \mathrm{S},-62.7819^{\circ} \mathrm{W}\right), 225 \mathrm{~m}$ asl, col. von Ellenrieder, 30 November 2007, NvE.

SAL 2: NR 81 ponds with riparian vegetation $\left(-23.4519^{\circ} \mathrm{S},-62.9319^{\circ} \mathrm{W}\right), 240 \mathrm{~m}$ asl, col. von Ellenrieder, 30 November 2007, NvE.

SAL 3: Unnamed stream $15 \mathrm{~km}$ SE of Isla de Cañas $\left(-22.9256^{\circ} \mathrm{S},-64.5758^{\circ} \mathrm{W}\right), 706 \mathrm{~m}$ asl, col. von Ellenrieder, 8 December 2008, CSCA.

SAL 4: Unnamed stream $20 \mathrm{~km} \mathrm{SE}$ of Isla de Cañas $\left(-22.9583^{\circ} \mathrm{S},-64.5556^{\circ} \mathrm{W}\right), 661 \mathrm{~m}$ asl, col. von Ellenrieder, 8 December 2008, CSCA.

SFE 1: Del Medio stream, $3 \mathrm{~km} \mathrm{~S}$ of General Gelly (approx. $9 \mathrm{~km} \mathrm{~N}$ of Mariano Benítez) $\left(-33.6172^{\circ} \mathrm{S},-60.5842^{\circ} \mathrm{W}\right), 53 \mathrm{~m}$ asl, col. Muzón, Lozano \& Ramos, 18 February 2016.

SFE 2: Capitán Bermúdez $\left(-32.8167^{\circ} \mathrm{S},-60.7167^{\circ} \mathrm{W}\right), 3 \mathrm{~m}$ asl, col. Macía, February 1984.

SFE 3: Garay, Leyes River $\left(-31.4638^{\circ} \mathrm{S},-60.5404^{\circ} \mathrm{W}\right), 18 \mathrm{~m}$ asl, col. Manzo, Manzo DataBase.

SFE 4: Garay, Delta of the Leyes River $\left(-31.4602^{\circ} \mathrm{S},-60.5492^{\circ} \mathrm{W}\right), 15 \mathrm{~m}$ asl, col. Manzo, 23 March 2015, Manzo DataBase.

SFE 5: $\quad$ La Capital, El Escondido $\left(-31.6492^{\circ} \mathrm{S},-60.5743^{\circ} \mathrm{W}\right), 15 \mathrm{~m}$ asl, col. Manzo, $2013-$ 2015, Manzo Database.

SFE 6: La Capital, Del Medio lagoon $\left(-31.6397^{\circ} \mathrm{S},-60.4744^{\circ} \mathrm{W}\right), 16 \mathrm{~m}$ asl, col. Manzo, 2013-2015, Manzo Database.

SFE 7: La Capital, El Chajacito lagoon $\left(-31.6639^{\circ} \mathrm{S},-60.4933^{\circ} \mathrm{W}\right), 14 \mathrm{~m}$ asl, col. Manzo, 2013-2015, Manzo Database.

SFE 8: La Capital, La Chicana lagoon $\left(-31.7530^{\circ} \mathrm{S},-60.7633^{\circ} \mathrm{W}\right), 11 \mathrm{~m}$ asl, col. Manzo, 2013-2015, Manzo Database.

SFE 9: La Capital, La Ferranda lagoon $\left(-31.6435^{\circ} \mathrm{S},-60.5740^{\circ} \mathrm{W}\right), 12 \mathrm{~m}$ asl, col. Manzo, 2013-2015, Manzo Database.

SFE 10: La Capital, La Perla lagoon $\left(-31.6540^{\circ} \mathrm{S},-60.5283^{\circ} \mathrm{W}\right), 15 \mathrm{~m}$ asl, col. Manzo, $2013-$ 2015, Manzo Database.

SFE 11: La Capital, Las Garzas lagoon $\left(-31.7178^{\circ} \mathrm{S},-60.7276^{\circ} \mathrm{W}\right), 11 \mathrm{~m}$ asl, col. Manzo, 2013-2015, Manzo Database.

SFE 12: La Capital, Madrejón Don Felipe North Colastine $\left(-31.6564^{\circ} \mathrm{S},-60.6009^{\circ} \mathrm{W}\right), 10 \mathrm{~m}$ asl, col. Muzón et al., 5 April 2014.

SFE 13: La Capital, Recu lagoon Ciudad Universitaria $\left(-31.5746^{\circ} \mathrm{S},-60.5214^{\circ} \mathrm{W}\right), 16 \mathrm{~m}$ asl, col. Manzo, 2013-2015, Manzo Database.

SFE 14: La Capital, Reservorio Las Vacas, Leyes stream $\left(-31.5736^{\circ} \mathrm{S},-60.5250^{\circ} \mathrm{W}\right), 19 \mathrm{~m}$ asl, col. Manzo \& Manzo, 27 October 2013, Manzo Database.

SFE 15: La Capital, Reservorio UPCN North Colastine $\left(-31.6256^{\circ} \mathrm{S},-60.6152^{\circ} \mathrm{W}\right), 20 \mathrm{~m}$ asl, col. Manzo, 7 February 2015, Manzo Database.

SFE 16: La Capital, South Colastine $\left(-31.6613^{\circ} \mathrm{S},-60.6029^{\circ} \mathrm{W}\right), 9 \mathrm{~m}$ asl, col. MFA, Manzo Database.

SFE 17: La Capital, Ubajay Stream $\left(-31.5762^{\circ} \mathrm{S},-60.5114^{\circ} \mathrm{W}\right), 8 \mathrm{~m}$ asl, col. Manzo, 16 March 2014, Manzo Database.

SFE 18: La Capital, Urban Zone Santa Fe City $\left(-31.6167^{\circ} \mathrm{S},-60.7124^{\circ} \mathrm{W}\right), 15 \mathrm{~m}$ asl, col. Guitart (in light trap), Manzo Database.

SFE 19: San Jerónimo, La Chancha lagoon $\left(-31.8188^{\circ} \mathrm{S},-60.7083^{\circ} \mathrm{W}\right), 13 \mathrm{~m}$ asl, col. Manzo, 2013-2015, Manzo Database. 
SFE 20: San Jerónimo, Los Sauces lagoon $\left(-31.8372^{\circ} \mathrm{S},-60.7236^{\circ} \mathrm{W}\right), 29 \mathrm{~m}$ asl, col. Manzo, 2013-2015, Manzo Database.

SFE 21: Madrejón Don Felipe $\left(-31.6585^{\circ} \mathrm{S},-60.6012^{\circ} \mathrm{W}\right), 17 \mathrm{~m}$ asl, col. Muzón, Lozano, Ramos, del Palacio \& Manzo, 5 April 2014.

SFE 22: Recreo $\left(-31.5000^{\circ} \mathrm{S},-60.7333^{\circ} \mathrm{W}\right), 17 \mathrm{~m}$ asl, 20 December 1948 , RWG.

SGO 1: Surroundings of La Cañada stream $\left(-29.6857^{\circ} \mathrm{S},-62.6257^{\circ} \mathrm{W}\right), 79 \mathrm{~m}$ asl, col. Lozano \& Lambruschini, 11 February 2008.

SGO 2: Route $13,5 \mathrm{~km}$ from Las Abras towards Sumampa $\left(-29.2269^{\circ} \mathrm{S},-62.9602^{\circ} \mathrm{W}\right), 90 \mathrm{~m}$ asl, col. Lozano \& Lambruschini, 12 February 2008.

SGO 3: Route 13 towards Sumampa, flooded field by the route $\left(-29.2193^{\circ} \mathrm{S},-63.0705^{\circ} \mathrm{W}\right)$, $92 \mathrm{~m}$ asl, col. Lozano \& Lambruschini, 12 February 2008.

SGO 4: Route 152 to Villa Unión $\left(-29.3433^{\circ} \mathrm{S},-62.8035^{\circ} \mathrm{W}\right), 85 \mathrm{~m}$ asl, col. Lozano \& Lambruschini, 11 February 2008.

SGO 5: Termas de Río Hondo $\left(-27.5151^{\circ} \mathrm{S},-64.8907^{\circ} \mathrm{W}\right), 270 \mathrm{~m}$ asl SGO 5a: col. Fidalgo, 9 September 1971-16 October 1971, FML. SGO 5b: col. Muzón \& von Ellenrieder, 7 January 1997.

SLU 1: Bajo de Véliz, Rincón del Carmen stream $\left(-32.3126^{\circ} \mathrm{S},-65.4117^{\circ} \mathrm{W}\right), 650 \mathrm{~m}$ asl, col. Muzón, 16 November 2007.

SLU 2: Merlo, Municipal Reserve, El Molina stream $\left(-32.3533^{\circ} \mathrm{S},-64.9531^{\circ} \mathrm{W}\right), 1200 \mathrm{~m}$ asl, col. Muzón, 15-16 November 2007.

SLU 3: Potrero de los Funes $\left(-33.2347^{\circ} \mathrm{S},-66.2349^{\circ} \mathrm{W}\right), 962 \mathrm{~m}$ asl, col. del Palacio, 30 January 2015.

TUC 1: Departamento de Tafí, San Pedro de Colalao, Tacanas $\left(-26.2333^{\circ} \mathrm{S},-65.4833^{\circ} \mathrm{W}\right)$, $1056 \mathrm{~m}$ asl, NvE Yungas Database.

TUC 2: Tafí del Valle $\left(-26.8667^{\circ} \mathrm{S},-65.6833^{\circ} \mathrm{W}\right), 2014 \mathrm{~m}$ asl, NvE Yungas Database.

\section{Results}

The following list includes a total of 282 species, grouped in 86 genera and 14 families.

ANISOPTERA

AESHNIDAE

Anax amazili (Burmeister, 1839): [LC]; BUE, CAT (Rodríguez, Gómez, \& Molineri, 2014), CHA, COR, ENT, LAP, MIS, SAL, SFE, SGO, TUC (CHH; CHS; CYM; DIP; ESP; EST; PAM; SEL; YUN) — L \{Calvert, 1934; Rodrigues Capitulo, 1981\}.

Anax concolor Brauer, 1865: [LC]; MIS (SEL) — L \{Geijskes, 1968\}.

Andaeschna rufipes (Ris, 1918): [LC]; JUJ (YUN) — L \{De Marmels, 1982a, 1992a\}.

Castoraeschna decurvata Dunkle \& Cook, 1984: [NA]; CBA, ENT (CHS; ESP; PAM) — L

\{Rodrigues Capitulo \& Jurzitza, 1989\}.

Castoraeschna januaria (Hagen, 1867): [NA]; MIS (SEL) — L \{Not described .

Coryphaeschna adnexa (Hagen, 1861): [LC]; BUE (8b, 9), CHA, COR, ENT, FOR (von Ellenrieder, 2010), JUJ, MIS, SAL, SFE, TUC (CHH; CHS; CYM; DIP; ESP; EST; PAM; YUN) — L \{Calvert, 1956; Santos, 1970a\}.

Coryphaeschna perrensi (McLachlan, 1887): [NA]; BUE, CBA, COR, JUJ, MIS, SFE, TUC (Rodríguez, Gómez, \& Molineri, 2018) (CHS; CYM; DIP; ESP; EST; PAM; SEL; YUN) — L \{Carvalho, 1992, 1993; Santos, 1969a\}.

Gynacantha adela Martin, 1909: [NA]; JUJ, MIS, SAL, TUC (Rodríguez \& Molineri, 2013) (SEL; YUN) - L \{Not described $\}$. 
Gynacantha bifida Rambur, 1842: [NA]; BUE, COR, JUJ, MIS, SFE, TUC (DIP; EST; PAM; SEL; YUN) — L \{Carvalho, 1987\}.

Gynacantha convergens Förster, 1908: [NA]; CHA (von Ellenrieder, 2010), JUJ, SFE (16, 18), TUC (Rodríguez et al., 2018) (CHS; DIP; YUN) — L \{Not described .

Gynacantha gracilis (Burmeister, 1839): [NA]; MIS (SEL) — L \{Santos, 1973a\}.

Gynacantha mexicana Selys, 1868: [LC]; SAL (Rodríguez et al., 2018) (YUN) — L \{Carvalho \& Ferreira, 1989\}.

Limnetron antarcticum Förster, 1907: [NA]; MIS (SEL) — L \{del Palacio \& Muzón, 2014\}.

Remartinia luteipennis (Burmeister, 1839): [LC]; JUJ, MIS, SAL, TUC (Rodríguez \& Molineri, 2013) (SEL; YUN) - L \{Calvert, 1956: only $R$. luteipennis florida\}. There are three subspecies described: $R$. luteipennis florida, $R$. luteipennis luteipennis, and $R$. luteipennis peninsularis; in Argentina only R. luteipennis luteipennis has been recorded.

Rhionaeschna absoluta (Calvert, 1952): [NA]; BUE, CAT, CHU, CBA, ENT, JUJ, LAP, LAR, MEN, NEU, RIO, SAL, SJU, SLU (1), SCR, SFE, SGO, TUC (ALT; BPA; CHS; DIP; EPA; ESP; MLM; MSB; PAM; PUN; YUN) — L \{von Ellenrieder, 2001a\}.

Rhionaeschna bonariensis (Rambur, 1842): [NA]; BUE, CAT, CHA, CBA, COR, ENT, FOR, JUJ, LAP (del Palacio, Diez, \& Latini, 2017), LAR, MEN, MIS, NEU (von Ellenrieder, 2001b), RIO, SAL, SJU, SLU (1), SFE, SGO, TUC (BPA; CHH; CHS; CYM; DIP; ESP; EST; MLM; MSB; PAM; SEL; YUN) — L \{Rodrigues Capitulo, 1980; von Ellenrieder, 2001a\}. The record from NEU (Lago Nahuel Huapi, Isla Victoria) was published by von Ellenrieder (2001b) but it was mistakenly assigned to RIO; therefore it did not appear in the checklist of 2008. The other record from RIO appears in von Ellenrieder (2001b): "Bahía San Matías" but the exact locality could not be georeferenced.

Rhionaeschna confusa (Rambur, 1842): [NA]; BUE, CBA, ENT, MEN, MIS, SFE, TUC (DIP; ESP; PAM; SEL; YUN) — L \{von Ellenrieder, 2001a\}.

Rhionaeschna diffinis (Rambur, 1842): [LC]; CHU, NEU, RIO (BPA; EPA) — L \{Calvert, 1956; von Ellenrieder, 2001a\}.

Rhionaeschna fissifrons (Muzón \& von Ellenrieder, 2001): [NA]; CAT, SAL (ALT; CHS; MSB) - L $\{$ Not described $\}$.

Rhionaeschna haarupi (Ris, 1908): [NA]; CAT, MEN, SAL, TUC (MLM; MSB; YUN) — L $\{$ Not described $\}$.

Rhionaeschna pallipes (Fraser, 1947): [NA]; BUE, CAT, CBA, LAR, MEN, SAL, SLU (2), SFE, TUC (CHS; DIP; ESP; MLM; MSB; PAM; YUN) — L \{von Ellenrieder \& Muzón, 2003a\}.

Rhionaeschna planaltica (Calvert, 1952): [LC]; BUE, CAT, CBA, ENT (1c, 1d, 6), JUJ, MIS, SAL, TUC (CHS; CYM; DIP; MSB; PAM; SEL; YUN) — L \{De Marmels, 1992b; von Ellenrieder, 1999\}.

Rhionaeschna psilus (Calvert, 1947): [LC]; SAL (YUN) — L \{Calvert, 1956; Needham \& Westfall, 1955\}.

Rhionaeschna variegata (Fabricius, 1775): [NA]; CAT, CHU, JUJ, LAR (Rodríguez et al., 2018), MEN, NEU, RIO, SAL, SCR, SJU (Rodríguez et al., 2018), TFU, TUC (ALT; BPA; EPA; MLM; MSB; PUN; YUN) - L \{Muzón \& von Ellenrieder, 1996; von Ellenrieder, 2001a\}.

Rhionaeschna vigintipunctata (Ris, 1918): [NA]; CAT, JUJ, LAR, SAL, TUC (CHS; MSB; YUN) — L \{Rodríguez \& Molineri, 2014\}.

Staurophlebia bosqi Navás, 1927: [EN B1ab(iii)]; BUE, ENT (Muzón, Lozano, del Palacio, Ramos, \& Lutz, 2015) (DIP; PAM) — L \{Bachmann, 1963\}. The record from ENT (Delta del Paraná, arroyo El Brasilero, Quinta Arco Iris) was published by Muzón et al. (2015) but it was mistakenly assigned to BUE.

Staurophlebia reticulata (Burmeister, 1839): [NA]; COR, ENT (1b, 1d, 1e), MIS (CYM; DIP; ESP; SEL) — L \{Geijskes, 1959: only S. reticulata reticulata\}. There are three subspecies 
described: S. reticulata guatemalteca, $S$. reticulata obscura, and S. reticulata reticulata; in Argentina only $S$. reticulata reticulata has been recorded.

Triacanthagyna caribbea Williamson, 1923: [LC]; JUJ (Rodríguez et al., 2018) (YUN) — L \{Santos, 1973b\}.

Triacanthagyna nympha (Navás, 1933): [NA]; BUE, CHA (Ris, 1913; von Ellenrieder, 2010; von Ellenrieder \& Garrison, 2003), COR, JUJ, MIS (CYM; DIP; PAM; SEL) — L \{ According to Garrison, von Ellenrieder, and Louton (2006) the larva was described by Carvalho (1988) as T. ditzleri\}. The record from Jujuy belongs to Fraser (1947); the single specimen was determined as Gynacantha trifida and no locality was mentioned.

\section{AUSTROPETALIIDAE}

Hypopetalia pestilens McLachlan, 1870: [LC]; CHU (Pessacq \& Brand, 2009), RIO (Pessacq \& Brand, 2009) (BPA) — L \{Pessacq \& Brand, 2009; Schmidt, 1941\}.

Phyllopetalia apollo Selys, 1878: [LC]; CHU (Pessacq \& Brand, 2009), RIO (Pessacq \& Brand, 2009) (BPA) — L \{Pessacq \& Brand, 2009\}.

Phyllopetalia pudu Dunkle, 1985: [LC]; CHU (von Ellenrieder, 2005), NEU, RIO (BPA) — L

$\{$ Not described\}.

\section{CORDULIIDAE}

Neocordulia setifera (Hagen in Selys, 1871): [NA]; MIS (SEL) — L \{Costa \& Santos, 2000\}. Rialla villosa (Rambur, 1842): [NA]; CHU, NEU, RIO (BPA; EPA) — L \{Needham \& Bullock, $1943\}$.

\section{GOMPHIDAE}

Aphylla dentata Selys, 1859: [NA]; FOR (von Ellenrieder \& Garrison, 2008a) (CHH; CHS) — L

\{According to Garrison et al. (2006) the larva was described by Belle (1964) as A. simulata\}. The record from Formosa was first published by von Ellenrieder \& Garrison (2008a) despite being mentioned as a first record in von Ellenrieder (2010).

Aphylla distinguenda Campion, 1920: [NA]; BUE, ENT, FOR (von Ellenrieder, 2010), MIS, SFE (CHH; CHS; CYM; DIP; PAM) - L \{Not described .

Aphylla producta Selys, 1854: [NA]; COR, MIS, SAL, SGO (CYM; ESP; SEL) — L \{Belle, 1964; Needham, 1944\}.

Aphylla theodorina (Navás, 1933): [LC]; COR, MIS, SAL (Rodríguez et al., 2014) (CYM; EST; SEL; YUN) - L \{Belle, 1992\}.

Archaeogomphus densus Belle, 1982: [NA]; MIS — L \{Not described\}.

Archaeogomphus infans (Ris, 1913): [NA]; MIS (Ris, 1913) (CYM) - L \{Not described $\}$. This species was originally described by Ris (1913) based on specimens from MIS (without exact locality) and Espirito Santo (Brazil). This species was never reported again from Argentina, and it was not included in the last checklist (von Ellenrieder \& Garrison, 2008a). In this contribution this species was recorded for MIS (3).

Cyanogomphus waltheri Selys, 1873: [LC]; MIS (CYM; SEL) - L \{Not described\}.

Epigomphus paludosus Hagen in Selys, 1854: [LC]; MIS, SGO (CHS; SEL) — L \{Costa, 1986\}. Gomphoides praevia St. Quentin, 1967: [NA]; MIS (SEL) — L \{Not described\}. Neogomphus edenticulatus Carle \& Cook, 1984: [NA]; CHU, NEU (BPA) — L \{Belle, 1992\}. Neogomphus molestus (Hagen in Selys, 1854): [NA]; CHU, NEU (BPA) — L \{Needham \& Bullock, 1943\}.

Phyllocycla argentina (Hagen in Selys, 1878): [NA]; BUE, CBA, COR, ENT (9), JUJ, MIS, SAL, SFE, SGO (Rodríguez et al., 2018), TUC (Rodríguez \& Molineri, 2013) (CHS; DIP; ESP; EST; PAM; SEL; YUN) — L \{Rodrigues Capitulo, 1983a\}. 
Phyllocycla basidenta Dunkle, 1987: [LC]; JUJ, SAL (YUN) — L \{Not described .

Phyllocycla foliata Belle, 1988: [NA]; MIS (Belle, 1988) (SEL) — L \{Not described\}.

This species was described by Belle from specimens collected by Jurzitza in Misiones

(Parque Nacional Iguazú). However, it was not listed by von Ellenrieder and Muzón (2008).

Phyllocycla propinqua Belle, 1972: [NA]; ENT (Belle, 1988), MIS (CYM; PAM; SEL) — L

\{Needham, 1940\}. This species was recorded by Belle (1988) from Entre Ríos. However, it was recorded only for Misiones by von Ellenrieder and Muzón (2008).

Phyllocycla vesta Belle, 1972: [NA]; BUE (PAM) — L \{Not described\}.

Phyllocycla viridipleuris (Calvert, 1909): [LC]; BUE (8a), CHA (4), COR (3d), ENT, MIS, SAL

(CHH; CYM; ESP; PAM; SEL) - L \{Belle, 1992\}.

Phyllogomphoides andromeda (Selys, 1869): [LC]; MIS (SEL) — L \{Belle, 1970\}.

Phyllogomphoides joaquini Rodrigues Capitulo, 1992: [VU B1ab(iii)]; BUE (PAM) — L

\{Muzón, Pessacq, \& von Ellenrieder, 2006\}.

Progomphus aberrans Belle, 1973: [NA]; CBA, COR, ENT, MIS (DIP; PAM) - L \{Not described\}.

Progomphus auropictus Ris, 1911: [NA]; MIS (SEL) — L \{Not described\}.

Progomphus australis Belle, 1973: [NA]; ENT (PAM) — L \{Not described\}.

Progomphus basistictus Ris, 1911: [NA]; MIS (SEL) — L \{Not described\}.

Progomphus complicatus Selys, 1854: [LC]; CAT (Rodríguez et al., 2014), JUJ, MIS, SAL, TUC

(CHS; SEL; YUN) — L \{Santos, 1968a\}.

*Progomphus costalis Hagen in Selys, 1854: [LC]; MIS (2a, 2b) (SEL) — L \{Not described\}.

Progomphus joergenseni Ris, 1908: [NA]; CAT, CBA, LAR (Rodríguez et al., 2014), MEN,

NEU, RIO, SAL, SJU, TUC (CHS; EPA; ESP; MLM; MSB; YUN) — L \{Muzón \& Lozano,

$2011\}$.

Progomphus kimminsi Belle, 1973: [NT]; JUJ, SAL, TUC (YUN) — L \{Not described\}.

Progomphus lepidus Ris, 1911: [NA]; MIS (SEL) — L \{Needham, 1941\}.

Progomphus phyllochromus Ris, 1918: [LC]; JUJ, SAL, SGO (Rodríguez et al., 2018), TUC

(CHS; YUN) - L \{Limongi, 1983\}.

Tibiagomphus noval (Rodrigues, 1985): [NA]; ENT (PAM) — L \{Rodrigues Capitulo, 1985\}.

Tibiagomphus uncatus (Fraser, 1947): [LC]; COR (3d, 3f), ENT, MIS (ESP; SEL) — L \{Not described\}.

Zonophora diversa Belle, 1983: [NA]; MIS (SEL) — L \{Not described\}.

\section{LIBELLULIDAE}

Brachymesia furcata (Hagen, 1861): [LC]; BUE (5a, 5b, 5c, 5d, 5e, 5f), COR, ENT, JUJ (Rodríguez et al., 2014), MIS, SAL, SGO, TUC (CHS; CYM; ESP; EST; PAM; SEL; YUN) — L \{Geijskes, 1934; Needham, Westfall, \& May, 2000\}.

Brachymesia herbida (Gundlach, 1889): [LC]; BUE (Ramos et al., 2017), CHA (1), COR, FOR (von Ellenrieder \& Garrison, 2008a), MIS, TUC (Rodríguez et al., 2018) (CHH; CHS; CYM; ESP; EST; PAM; SEL; YUN) - L \{Needham et al., 2000\}.

Brechmorhoga nubecula (Rambur, 1842): [NA]; JUJ, MIS, SAL, TUC (Rodríguez \& Molineri, 2013) (SEL; YUN) — L \{Santos, 1969b\}.

Brechmorhoga praedatrix Calvert, 1909: [LC]; MIS (SEL) — L \{Fleck, 2004\}.

Brechmorhoga vivax Calvert, 1906: [NA]; JUJ, MIS, LAR (Rodríguez et al., 2018), SAL, TUC (MSB; YUN) - L \{De Marmels, 1982b\}.

Cannaphila vibex (Hagen, 1861): [LC]; CAT, JUJ, SAL, TUC (CHS; YUN) — L \{Limongi, $1991\}$.

*Dasythemis esmeralda Ris, 1910: [NA]; COR (8a, 8b) (CYM) — L \{Not described\}. 
Dasythemis mincki (Karsch, 1890): [LC]; CBA, ENT, MIS, RIO, SAL, SLU (CHS; EPA; ESP; PAM; SEL; YUN) — L \{Carvalho, Werneck-de-Carvalho, \& Calil, 2002; von Ellenrieder, 2007c\}. There are two subspecies recognized, D. mincki mincki and D. mincki clara Ris, 1908, both of which have been found in Argentina. The former has been recorded only for MIS.

Dasythemis venosa (Burmeister, 1839): [NA]; COR (8a), MIS (CYM; SEL) — L \{Carvalho et al., 2002\}. Navás (1927b) mentions this species for CBA (Sierra de Cordoba) based on one female. This is likely to be a misidentification of $D$. mincki.

Diastatops intensa Montgomery, 1940: [NA]; CHA (2), CBA, COR, ENT, FOR (Montgomery, 1940; Ris, 1928; von Ellenrieder, 2010), MIS, SAL (von Ellenrieder, 2010), SFE (2, 12) (CHH; CHS; CYM; DIP; ESP; EST; PAM; SEL; YUN) — L \{Costa, Souza-Franco, \& Takeda, 1999\}. The first record from Formosa was by Ris (1928) as D. pullata; Montgomery (1940) in his revision assigned this record to $D$. intensa. This information was not included in the last checklist (von Ellenrieder \& Muzón, 2008). von Ellenrieder (2010) mentioned this species again for Formosa.

Diastatops obscura (Fabricius, 1775): [NA]; CBA, COR, MIS (CYM; EST; SEL) — L \{Santos, Costa, \& Pujol-Luz, 1993\}.

Diastatops pullata (Burmeister, 1839): [LC]; BUE, CHA, COR, SFE (DIP; EST) — L \{Fleck, $2003\}$.

Dythemis nigra Martin, 1897: [NA]; BUE, COR (2, 8b), JUJ, MIS, SAL, SLU, TUC (CHS; CYM; SEL; YUN) — L \{De Marmels (1982b) as Dythemis multipunctata .

Edonis helena Needham, 1905: [NA]; COR (EST) — L \{Not described\}.

Elasmothemis cannacrioides (Calvert, 1906): [NA]; JUJ, MIS, SAL, TUC (CHS; SEL; YUN) — L \{Westfall, 1988\}.

Elasmothemis constricta (Calvert, 1898): [NA]; MIS (SEL) — L \{Pujol-Luz, 1990\}.

Erythemis attala (Selys in Sagra, 1857): [LC]; BUE, CHA, COR, ENT, FOR, JUJ, MIS, SAL, SFE, TUC (Rodríguez \& Moliner, 2013) (CHH; CHS; CYM; DIP; EST; PAM; SEL; YUN) — L \{Rodrigues Capitulo, 1983b\}.

Erythemis carmelita Williamson, 1923: [LC]; FOR (von Ellenrieder, 2010) (CHH) - L \{Not described\}.

Erythemis credula (Hagen, 1861): [NA]; COR (CYM; ESP; EST) - L \{Calvert, 1928; Klots, 1932; Santos, 1969c\}.

Erythemis mithroides (Brauer in Therese, 1900): [LC]; CHA (Ris, 1913; von Ellenrieder, 2010), CBA, COR, FOR, MIS, SFE (CHH; DIP; EST; SEL) — L \{Costa \& Pujol-Luz, $1993\}$.

Erythemis peruviana (Rambur, 1842): [LC]; CHA, COR, ENT, FOR, MIS, SFE (21) (CHH; CHS; CYM; DIP; ESP; EST; PAM; SEL) — L \{Calvert, 1928; Klots, 1932\}.

Erythemis plebeja (Burmeister, 1839): [LC]; BUE, CHA, COR, ENT, FOR, JUJ (Rodríguez et al., 2014), LAR (Rodríguez et al., 2018), MIS, SAL, SFE, SGO, TUC (CHH; CHS; CYM; DIP; ESP; EST; PAM; SEL; YUN) — L \{Calvert, 1928; Klots, 1932; Needham \& Westfall, 1955\}.

Erythemis vesiculosa (Fabricius, 1775): [LC]; BUE, CAT (Ris, 1928), CHA (Ris, 1913; von Ellenrieder, 2010), CBA, COR, FOR, JUJ, LAP (del Palacio et al., 2017), MIS, SAL, SFE, SGO, TUC (CHH; CHS; CYM; DIP; ESP; EST; MSB; PAM; SEL; YUN) — L \{Klots, 1932; Needham \& Westfall, 1955\}.

*Erythrodiplax amazonica Sjöstedt, 1918: [NA]; MIS (9) (SEL) — L \{De Marmels, 1992b\}.

Erythrodiplax anomala (Brauer, 1865): [NA]; MIS - L \{Carvalho, Ferreira, \& Nessiminian, 1991\}. Navás (1922) mentioned this species for BUE; since this is likely to be a misidentification the record from BUE has been removed. 
Erythrodiplax atroterminata Ris, 1911: [NA]; BUE, CAT, CBA, COR, ENT (Rodrigues Capitulo \& Muzón, 1985), LAP (del Palacio et al., 2017), MIS, RIO, SAL, SJU, SLU, SGO (5a), TUC (1, 2) (CHS; CYM; EPA; ESP; EST; MLM; MSB; PAM; SEL; YUN) — L \{Garré, Muzón, \& Ardohain, 2008\}.

Erythrodiplax basalis (Kirby, 1897): [NA]; MIS, SGO (CHS; SEL) — L \{Costa, Vieira, \& Lourenço, 2001\}.

Erythrodiplax castanea (Burmeister, 1839): [NA]; MIS (SEL) — L \{Not described\}.

Erythrodiplax chromoptera Borror, 1942: [NA]; BUE (del Palacio \& Muzón, 2019), COR, ENT (del Palacio \& Muzón, 2019), MIS (CYM; ESP; PAM) — L \{Not described\}.

Erythrodiplax connata (Burmeister, 1839): [NA]; CHU, MEN (1, 2, 3, 4a, 4b), NEU, RIO (ALT; BPA; EPA; ESP; MLM; MSB) — L \{Lozano, Muzón, \& del Palacio, 2011\}.

Erythrodiplax corallina (Brauer, 1865): [NA]; BUE, CAT, CHU, CBA, COR, JUJ, LAP, LAR, MEN, NEU, RIO, SAL, SJU, SLU (1), SFE (Förster, 1914; Borror, 1942), SGO, TUC (ALT; CHH; CHS; CYM; DIP; EPA; EST; MLM; MSB; PAM; YUN) — L \{Garré et al., 2008\}.

Erythrodiplax famula (Erichson in Schomburgk, 1848): [NA]; MIS — L \{Not described\}.

Erythrodiplax fusca (Rambur, 1842): [LC]; BUE, CHA, CBA (Navás, 1928), COR, ENT, MIS,

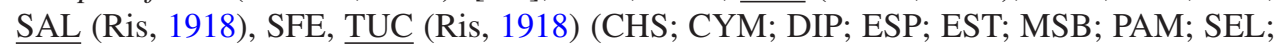
YUN) - L $\{$ Santos, 1967\}.

Erythrodiplax juliana Ris, 1911: [LC]; MIS (SEL) — L \{Carvalho et al., 1991\}.

Erythrodiplax latimaculata Ris, 1911: [NA]; MIS (SEL) — L \{Costa et al., 2001\}.

Erythrodiplax lativittata Borror, 1942: [NA]; MIS — L \{Not described\}.

Erythrodiplax lygaea Ris, 1911: [LC]; COR (5), MIS (EST; SEL) — L \{Costa et al., 2001\}.

Erythrodiplax media Borror, 1942: [NA]; BUE (2, 3, 4, 5 g, 8b), CHA (1), COR, ENT, FOR (von Ellenrieder, 2010), JUJ, MIS, RIO, SAL, TUC (CHS; CYM; DIP; EPA; ESP; EST; PAM; SEL; YUN) — L \{Dalzochio, Périco, Renner, \& Sahlén, 2018\}.

Erythrodiplax melanorubra Borror, 1942: [NA]; BUE, COR, ENT, JUJ, LAR (Rodríguez et al., 2014), MIS, SAL, SGO, TUC (CHS; CYM; DIP; MSB; PAM; SEL; YUN) — L \{Limongi, $1991\}$.

Erythrodiplax nigricans (Rambur, 1842): [NA]; BUE, CAT, CHA, CBA (Zapata \& Pereyra, 2016), COR, ENT, FOR (von Ellenrieder, 2010), JUJ (Rodríguez et al., 2014), LAP (del Palacio et al., 2017), LAR, MEN, MIS, NEU, RIO, SAL (von Ellenrieder, 2010), SFE, SGO, TUC (Rodríguez \& Molineri, 2013) (ALT; CHH; CHS; CYM; DIP; ESP; EST; MLM; MSB; PAM; SEL; YUN) — L \{von Ellenrieder \& Muzón, 2000\}.

Erythrodiplax ochracea (Burmeister, 1839): [LC]; BUE, CHA, COR, FOR, MEN (Navás, 1924), MIS, NEU, SFE, SGO (Navás, 1922; von Ellenrieder, 2010) (ALT; CHH; CHS; CYM; DIP; EPA; ESP; EST; PAM; SEL) — L \{Carvalho et al., 1991\}.

Erythrodiplax pallida (Needham, 1904): [LC]; BUE (Muzón \& von Ellenrieder, 1998, as Erythrodiplax sp.; del Palacio \& Muzón, 2016), COR (von Ellenrieder \& Muzón, 2008, as Erythrodiplax sp. 1; del Palacio \& Muzón, 2016), ENT (von Ellenrieder \& Muzón, 2008, as Erythrodiplax sp. 1) (DIP; ESP; EST; PAM) - L \{Costa et al., 2001\}.

Erythrodiplax paraguayensis (Förster, 1905): [LC]; BUE, CHA, CBA, COR, ENT, FOR, MIS, SAL (1, 2) (CHH; CHS; CYM; DIP; ESP; EST; PAM; SEL) — L \{Muzón \& Garré, 2005\}. Erythrodiplax umbrata (Linnaeus, 1758): [LC]; BUE, CAT, CHA, CBA (Zapata \& Pereyra, 2016), COR, ENT, FOR, JUJ, LAR, MIS, SAL, SFE, SGO (Navás, 1922; von Ellenrieder, 2010), TUC (CHH; CHS; CYM; DIP; ESP; EST; MSB; PAM; SEL; YUN) — L \{Calvert, 1928; Costa et al., 2001\}.

*Erythrodiplax unimaculata (De Geer, 1773): [NA]; MIS (12) (SEL) — L \{Not described\}.

Idiataphe longipes (Hagen, 1861): [NA]; COR (CYM; EST) — L \{Oldrini \& Mascarenhas, $2005\}$. 
Libellula herculea Karsch, 1889: [NA]; JUJ, MIS, SAL, TUC (Rodríguez \& Molineri, 2013) (SEL; YUN) — L \{De Marmels, 1982b, 1992b\}.

Macrothemis declivata Calvert, 1909: [NA]; MIS (SEL) — L \{Salgado et al., 2013\}.

Macrothemis hahneli Ris, 1913: [LC]; JUJ, SAL, TUC (CHS; YUN) — L \{von Ellenrieder, 2007c\}.

Macrothemis hemichlora (Burmeister, 1839): [LC]; MIS, SAL (Rodríguez et al., 2018) (SEL; YUN) — L \{Salgado et al., 2013\}.

Macrothemis heteronycha (Calvert, 1909): [LC]; COR (CYM; EST) - L \{Costa, Carriço, Santos, \& Mascarenhas, 2010\}.

Macrothemis imitans Karsch, 1890: [LC]; CAT, CBA, COR, ENT, JUJ, LAR (Rodríguez et al., 2014), MIS, SAL, SGO, TUC (CHS; CYM; ESP; MSB; PAM; SEL; YUN) — L \{ Salgado et al., 2013\}.

Macrothemis inacuta Calvert, 1898: [LC]; CHA (2), COR (3e), FOR, JUJ (Rodríguez et al., 2018), SAL (CHH; CHS; ESP) — L \{Novelo Gutiérrez \& Ramírez, 1998\}.

Macrothemis marmorata Hagen, 1868: [NA]; MIS (SEL) — L \{Not described\}.

Macrothemis musiva Calvert, 1898: [NA]; JUJ (Rodríguez et al., 2014), MIS, SAL (SEL; YUN) — L $\{$ Santos, 1970b\}.

Macrothemis polyneura Ris, 1913: [NA]; MIS (SEL) — L \{Not described\}.

Macrothemis tenuis Hagen, 1868: [LC]; MIS (SEL) — L \{Salgado et al., 2013\}.

Macrothemis tessellata (Burmeister, 1839): [NA]; BUE, MIS — L \{Not described\}.

Miathyria marcella (Selys in Sagra, 1857): [LC]; BUE, CHA, CBA (Navás, 1922; Zapata \& Pereyra, 2016), COR, ENT, FOR, JUJ (Rodríguez et al., 2014), LAP (del Palacio et al., 2017), LAR, MIS, SAL, SLU (1), SFE, SGO, TUC (CHH; CHS; CYM; DIP; ESP; EST; PAM; SEL; YUN) — L \{Bick, 1953; Klots, 1932; Westfall, 1953\}.

Micrathyria artemis Ris, 1911: [LC]; MIS (CYM; SEL) — L \{Santos, 1972b\}.

Micrathyria athenais Calvert, 1909: [NA]; ENT, MIS (PAM; SEL) — L \{Not described\}.

Micrathyria atra (Martin, 1897): [LC]; MIS, SAL (SEL; YUN) — L \{Santos, 1978\}.

Micrathyria catenata Calvert, 1909: [LC]; COR, JUJ, MIS, SAL (CYM; SEL; YUN) — L \{Not described\}.

Micrathyria dido Ris, 1911: [NA]; MIS (SEL) — L \{Not described\}.

Micrathyria eximia Kirby, 1897: [NA]; COR (DIP) — L \{Not described\}.

Micrathyria hesperis Ris, 1911: [NA]; CHA, COR, ENT, FOR (von Ellenrieder \& Garrison, 2008a), JUJ (Rodríguez et al., 2014), MIS, SAL, SGO, TUC (CHS; CYM; DIP; ESP; PAM; SEL; YUN) — L \{de Assis \& Costa, 1994\}.

Micrathyria hypodidyma Calvert, 1906: [NA]; BUE, CHA, COR, ENT, FOR, JUJ, MIS, SAL, SFE (Navás, 1917), TUC (CHH; CYM; DIP; ESP; EST; PAM; SEL; YUN) — L \{Santos, $1968 \mathrm{~b}\}$.

*Micrathyria iheringi Santos, 1946: [NA]; COR (8b), MIS (14b) (CYM; SEL) — L \{Not described\}.

Micrathyria longifasciata Calvert, 1909: [LC]; BUE, CHA, COR, ENT, FOR, JUJ, MEN, MIS (2c), SAL, SFE, SGO, TUC (CHH; CHS; CYM; DIP; ESP; EST; PAM; SEL; YUN) — L \{Souza \& Costa, 2002\}.

Micrathyria ocellata Martin, 1897: [NA]; CHA (1), JUJ, MIS (Jurzitza, 1981), SAL, TUC (Rodríguez et al., 2018) (CHS; SEL; YUN) — L \{de Assis \& Costa, 1994\}. There are two subspecies recognized, M. ocellata ocellata and M. ocellata dentiens Calvert, 1909. Only the latter has been found in Argentina.

Micrathyria pseudeximia Westfall, 1992: [NA]; COR, MIS (14b, 18b) (SEL) — L \{Dalzochio, 2009a\}.

Micrathyria ringueleti Rodrigues Capitulo, 1988: [NA]; BUE (DIP; PAM) — L \{Not described\}. 
Micrathyria spuria (Selys in Therese, 1900): [LC]; COR, ENT (CYM; ESP; EST; PAM) — L \{Souza \& Costa, 2002\}.

*Micrathyria stawiarskii Santos, 1953: [LC]; MIS (14b) (SEL) — L \{de Assis \& Costa, 1994\}. Micrathyria tibialis Kirby, 1897: [LC]; COR, FOR, SAL (von Ellenrieder, 2010), SFE (13, 17) (CHH; DIP; EST) - L \{Souza \& Costa, 2002\}.

Micrathyria ungulata Förster, 1907: [NA]; BUE, CBA, ENT, JUJ, MIS, SAL, SLU (CHS; PAM; SEL; YUN) — L \{Garré \& Lozano, 2007\}.

Micrathyria venezuelae De Marmels, 1989: [NA]; JUJ, MIS (14a, 15), SAL, TUC (Rodríguez \& Molineri, 2013) (SEL; YUN) - L \{Not described.

Nephepeltia aequisetis Calvert, 1909: [NA]; COR, FOR, MIS (1) (CHH; CYM; ESP) — L \{Not described\}.

Nephepeltia berlai Santos, 1950: [NA]; COR (8a, 8b), MIS (von Ellenrieder, 2014) (CYM; SEL) - L \{Dalzochio, 2009b\}.

Nephepeltia flavifrons (Karsch, 1889): [NA]; BUE (10), COR, ENT (5) (CYM; ESP; EST; PAM) - L $\{$ Not described $\}$.

Nephepeltia leonardina Rácenis, 1953: [LC]; SAL (Rodríguez et al., 2014) (YUN) — L \{Not described\}.

Nephepeltia phyrne (Perty, 1833): [LC]; MIS (SEL) — L \{De Marmels, 1990\}.

Oligoclada haywardi Fraser, 1947: [NA]; COR (8a), MIS (CYM; SEL) — L \{Not described\}. This species was only known from its type locality (MIS: Puerto Iguazú); in this contribution it has been rediscovered in MIS $(8,16)$.

Oligoclada laetitia Ris, 1911: [NA]; BUE (10), COR (3e, 8b), ENT, FOR, MIS, SAL (von Ellenrieder \& Garrison, 2008a) (CHS; CYM; DIP; ESP; PAM; SEL; YUN) - L \{Souza, Costa, \& Espindola, 2002\}.

Oligoclada rubribasalis von Ellenrieder \& Garrison, 2008: [NA]; COR (1a, 5, 6a, 6b), ENT (von Ellenrieder \& Garrison, 2008a), FOR (von Ellenrieder \& Garrison, 2008a), SFE (5, 6, 7, 8, 9, 10, 11, 13, 19, 20) $(\mathrm{CHH}$; DIP; EST) - L \{Not described $\}$.

Orthemis aequilibris Calvert, 1909: [NA]; SAL (YUN) — L \{Fleck, 2003\}.

Orthemis ambinigra Calvert, 1909: [NA]; BUE, COR, ENT (1c, 1d, 4b, 9), MIS, SAL (von Ellenrieder, 2012) (DIP; ESP; PAM; SEL; YUN) — L \{Not described\}.

Orthemis ambirufa Calvert, 1909: [NA]; COR, ENT, MIS (PAM) — L \{Not described\}.

Orthemis cultriformis Calvert, 1899: [NA]; MIS (SEL) — L \{Carvalho \& Werneck-de-Carvalho, $2005\}$.

Orthemis discolor (Burmeister, 1839): [LC]; BUE, CAT, CHA, $\underline{C B A}$ (Navás, 1922), COR, JUJ, MEN, MIS, SAL, SLU, SFE, TUC (CYM; DIP; EST; PAM; SEL; YUN) - L \{Not described\}.

Orthemis nodiplaga Karsch, 1891: [LC]; BUE, CAT, CHA, CBA (Zapata \& Pereyra, 2016), COR, ENT, FOR, LAP (del Palacio et al., 2017), MEN, MIS, SAL, SLU, SFE, SGO, TUC (CHH; CHS; CYM; DIP; ESP; EST; MSB; PAM; YUN) — L \{Rodrigues Capitulo \& Muzón, 1990\}.

Orthemis phillipi von Ellenrieder, 2009: [NA]; SAL (von Ellenrieder, 2010) (CHS) — L \{Not described\}.

Pantala flavescens (Fabricius, 1798): [LC]; BUE, CAT, CBA (Navás, 1922; Zapata \& Pereyra, 2016), COR, ENT, FOR, JUJ, LAP (del Palacio et al., 2017), MEN, MIS, SAL, SLU, SFE, SGO (1, 2, 3, 4), TUC (CHH; CHS; CYM; DIP; ESP; EST; MSB; PAM; SEL; YUN) — L \{Byers, 1941; Cabot, 1890; Geijskes, 1934\}.

Pantala hymenaea (Say, 1840): [LC]; CAT, CBA (Zapata \& Pereyra, 2016), LAP (2), LAR, MEN, MIS, SGO (Ris, 1913; von Ellenrieder, 2010), TUC (CHS; ESP; MSB; PAM; SEL; YUN) - L \{Kennedy, 1923\}.

Perithemis icteroptera (Selys in Sagra, 1857): [LC]; BUE, ENT, MIS, SAL (CYM; DIP; PAM; SEL; YUN) — L \{von Ellenrieder \& Muzón, 1999\}. 
Perithemis lais (Perty, 1833): [LC]; COR, MIS (CYM; ESP; SEL) — L \{Costa \& Régis, 2005\}.

Perithemis mooma Kirby, 1889: [NA]; BUE, CBA, COR, ENT, FOR (von Ellenrieder \& Garrison, 2008a), JUJ, MIS, SAL, SLU (1), SFE, SGO, TUC (CHH; CHS; CYM; DIP; ESP; EST; PAM; SEL; YUN) — L \{Santos, 1973c; von Ellenrieder \& Muzón, 1999\}.

Perithemis thais Kirby, 1889: [NA]; COR (1b, 8b), MIS (CYM; EST; SEL) — L \{Spindola, Souza, \& Costa, 2001\}.

Planiplax erythropyga (Karsch, 1891): [LC]; BUE, COR (Lozano, Muzón, \& Scattolini, 2012), ENT (ESP; EST; PAM) — L \{Not described\}.

Sympetrum gilvum (Selys, 1884): [NA]; CAT, CBA, JUJ, SAL, SLU, TUC (CHS; YUN) — L \{Limongi, 1991\}.

Sympetrum villosum Ris, 1911: [NA]; CHU, NEU, RIO (BPA) — L \{Muzón \& von Ellenrieder, 1997\}.

Tauriphila argo (Hagen, 1869): [LC]; BUE (Navás, 1930), COR, MIS, $\underline{S A L}$ (von Ellenrieder, 2010) (ESP; EST; PAM) — L \{Costa \& de Assis, 1994?; Fleck, Brenk, \& Misof, 2006\}.

Tauriphila risi Martin, 1896: [NA]; BUE, CHA, CBA, COR, ENT, FOR (von Ellenrieder \& Garrison, 2008a), LAP (del Palacio et al., 2017), SAL (von Ellenrieder, 2010), SFE, SGO, TUC (CHH; CHS; CYM; DIP; ESP; EST; PAM; YUN) — L \{Rodrigues Capitulo, 1996\}.

Tauriphila xiphea Ris, 1913: [NA]; COR, SAL (von Ellenrieder, 2010) (EST) — L \{Not described\}.

Tholymis citrina Hagen, 1867: [LC]; JUJ, SAL, SFE (13, 14, 17), TUC (ALT; DIP; YUN) — L \{Fleck, De Marmels, \& Grand, 2004\}.

Tramea abdominalis (Rambur, 1842): [LC]; JUJ, MIS, SAL (SEL; YUN) — L \{Cabot, 1890; Klots, 1932\}.

Tramea binotata (Rambur, 1842): [LC]; COR, MIS, SAL, $\underline{\text { SGO }}$ (Navás, 1922), $\underline{\text { SFE }}$ (Navás, 1930) (EST; SEL; YUN) — L \{Needham et al., 2000\}.

Tramea calverti Muttkowski, 1910: [LC]; CAT, COR, FOR (von Ellenrieder, 2010), JUJ, MIS, SAL (CHS; CYM; EST; YUN) - L \{Souza, Costa, \& Santos, 1999\}.

Tramea cophysa Hagen, 1867: [NA]; BUE, CAT, CHA (1, 2, 3, 5), COR, ENT, FOR, LAP (del Palacio et al., 2017), MIS, SAL, SFE (4, 13, 15, 21), SGO, TUC (CHH; CHS; CYM; DIP; ESP; EST; PAM; SEL; YUN) — L \{Santos, 1968c $\}$.

Tramea rustica De Marmels \& Rácenis, 1982: [LC]; MIS (SEL) — L \{Not described\}.

Uracis imbuta (Burmeister, 1839): [NA]; BUE, MIS (CYM) — L \{Not described\}. Rambur

(1842) recorded Uracis quadra (= Uracis imbuta) from "Buenos Ayres" without exact locality, but no other specimen of Uracis has been collected again in Buenos Aires.

Zenithoptera lanei Santos, 1941: [NA]; MIS (SEL) — L \{Not described\}.

\section{NEOPETALIIDAE}

Neopetalia punctata (Hagen in Selys, 1854): [DD]; NEU (BPA) — L \{Carle \& Louton, 1994\}.

PETALURIDAE

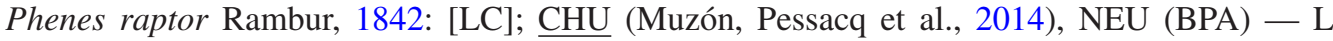
\{Schmidt, 1941; Needham \& Bullock, 1943\}.

\section{SYNTHEMISTIDAE}

Gomphomacromia fallax McLachlan, 1881: [LC]; SAL (YUN) — L \{Not described\}.

Gomphomacromia nodisticta Ris, 1928: [NA]; CAT, SAL, TUC (Rodríguez \& Molineri, 2013) (MSB; YUN) - L \{Not described .

Gomphomacromia paradoxa Brauer, 1864: [NA]; CHU, NEU, RIO (BPA; EPA) — L \{Theischinger \& Watson, 1984\}. 
ZYGOPTERA

\section{CALOPTERYGIDAE}

Hetaerina longipes Hagen in Selys, 1853: [NA]; MIS (SEL) — L \{Not described\}.

Hetaerina mendezi Jurzitza, 1982: [DD]; MIS (SEL) — L \{von Ellenrieder, 2007a\}.

Hetaerina proxima Selys, 1853: [NA]; MIS (SEL) — L \{Not described\}.

Hetaerina rosea Selys, 1853: [NA]; BUE, CBA, COR, ENT, JUJ, MIS, SAL, SFE (1, 4), SGO, TUC (CHS; CYM; DIP; ESP; EST; PAM; SEL; YUN) — L \{Pessacq \& Muzón, 2004; von Ellenrieder, 2007a\}.

Hetaerina sanguinea Selys, 1853: [NA]; SAL (YUN) — L \{Not described\}.

Mnesarete grisea (Ris, 1918): [NA]; CAT, JUJ, LAR, SAL, TUC (CHS; MSB; YUN) — L

\{Garrison, 2006\}.

Mnesarete guttifera (Selys, 1873): [LC]; MIS (CYM) - L \{Not described\}.

Mnesarete lencionii Garrison, 2006: [NA]; MIS (CYM) — L \{Not described\}.

Mnesarete pruinosa (Hagen in Selys, 1853): [NA]; MIS (SEL) — L \{Not described .

Mnesarete pudica (Hagen in Selys, 1853): [NA]; MIS (CYM; SEL) — L \{Guillermo-Ferreira \& Bispo, 2012\}.

\section{COENAGRIONIDAE}

Acanthagrion aepiolum Tennessen, 2004: [NA]; COR, ENT, JUJ (Rodríguez et al., 2018), MIS, SAL (CHS; CYM; ESP; EST; PAM; SEL; YUN) — L \{Lozano, Garré, \& Pessacq, 2007\}.

Acanthagrion ascendens Calvert, 1909: [NA]; COR, MIS? (CYM; SEL?) — L \{Geijskes, 1941\}. von Ellenrieder and Muzón (2008) removed this species from the checklist of Argentina based on the supposition of an allopatric distribution with A. aepiolum. However, Lozano (2013) found that some specimens from COR province were actually this species. Therefore, previous records of $A$. ascendens from MIS (Jurzitza, 1981) which were removed from the checklist by von Ellenrieder and Muzón (2008) were included with doubts here since its presence in COR makes its presence in MIS likely.

Acanthagrion cuyabae Calvert, 1909: [LC]; BUE (6, 7), CHA (1, 3, 4), CBA (2), COR, ENT, FOR, MIS, SAL (von Ellenrieder, 2010) (CHH; CHS; CYM; DIP; ESP; EST; PAM; SEL) - L $\{$ Not described $\}$.

Acanthagrion floridense Fraser, 1946: [LC: as A. peruvianum]; CBA, JUJ, MIS (Lozano, Rodríguez, \& Molineri, 2017), SAL, SLU (Lozano et al., 2017), SGO, TUC (CHS; ESP; SEL; YUN) - L \{Lozano et al., 2017\}.

Acanthagrion gracile (Rambur, 1842): [NA]; COR, ENT, MIS, SLU (1) (CHS; CYM; ESP; PAM; SEL) - L \{Anjos Santos et al., 2011\}.

Acanthagrion hildegarda Gloger, 1967: [NA]; BUE, CBA, ENT, MIS, SFE (CHS; DIP; ESP; PAM; SEL) — L \{Muzón, von Ellenrieder, \& Pessacq, 2001\}.

Acanthagrion lancea Selys, 1876: [NA]; BUE, CHA, CBA (Zapata \& Pereyra, 2016), COR, ENT, FOR (von Ellenrieder, 2010), JUJ, MIS, SAL, SFE, SGO (von Ellenrieder, 2010), TUC (CHH; CHS; CYM; DIP; ESP; EST; PAM; SEL; YUN) - L \{Anjos Santos et al., $2011\}$.

Acanthagrion minutum Leonard, 1977: [NA]; COR (CYM; EST) — L \{Not described\}. Acanthagrion temporale Selys, 1876: [NA]; MIS (SEL) — L \{Not described\}.

*Aceratobasis nathaliae (Lencioni, 2004): [NA]; MIS (7) (SEL) — L \{Not described\}. Aeolagrion philipi Tennessen, 2009: [NA]; CHA (3), FOR (von Ellenrieder, 2010) (CHH) — L $\{$ Not described $\}$.

Andinagrion garrisoni von Ellenrieder \& Muzón, 2006: [NT]; JUJ, SAL, SGO (Rodríguez et al., 2018), TUC (CHS; MSB; YUN) — L \{von Ellenrieder \& Muzón, 2006\}. 
Andinagrion peterseni (Ris, 1908): [NA]; BUE, CAT, CHU, LAP (del Palacio et al., 2017), MEN,

NEU, RIO, SAL, TUC (ALT; BPA; EPA; MLM; MSB; PAM) — L \{Bulla, 1973a\}.

Andinagrion saliceti (Ris, 1904): [NA]; BUE (PAM) — L \{Not described\}.

Antiagrion grinbergsi Jurzitza, 1974: [LC]; NEU (BPA) — L \{Not described\}.

Argentagrion ambiguum (Ris, 1904): [LC]; BUE, CAT (Ris, 1918), CHA, COR, ENT, FOR,

MIS, SAL, SFE, TUC (CHH; CHS; CYM; DIP; ESP; EST; MSB; PAM; SEL; YUN) — L

\{Bulla, 1970\}.

Argia albistigma Hagen in Selys, 1865: [NA]; ENT, MIS (SEL) — L \{Not described\}.

Argia croceipennis Selys, 1865: [LC]; ENT (6), MIS (CYM; DIP; SEL) — L \{Costa, Ravanello,

\& Souza-Franco, 2008\}.

Argia hasemani Calvert, 1909: [NA]; MIS (SEL) — L \{Not described\}.

Argia joergenseni Ris, 1913: [NA]; CAT, CBA, JUJ, LAR (Rodríguez et al., 2018), SAL, SGO

(Rodríguez et al., 2018), SJU, SLU, SFE (22), TUC (CHS; ESP; MSB; YUN) - L \{von

Ellenrieder, 2007b\}.

Argia jujuya Ris, 1913: [NA]; CAT, JUJ, SAL, TUC (YUN) — L \{Molineri \& Rodríguez, 2013\}. Argia lilacina Selys, 1865: [NA]; MIS (SEL) - L \{Not described .

Argia mollis Hagen in Selys, 1865: [NA]; MIS (SEL) — L \{Not described\}.

Argia reclusa Selys, 1865: [NA]; MIS (CYM; SEL) — L \{Not described\}.

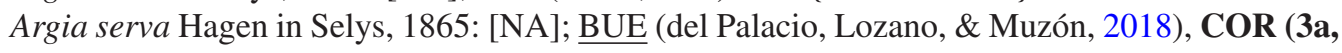

3b, 3c), ENT (3, 4a), MIS (DIP; ESP; PAM; SEL) — L \{del Palacio et al., 2018\}.

*Argia tamoyo Calvert, 1909: [NA]; MIS (10, 13) (SEL) — L \{Not described\}.

Argia translata Hagen in Selys, 1865: [LC]; JUJ, SAL (CHS; YUN) — L \{Geijskes, 1946; von

Ellenrieder, 2007b\}.

Argia yungensis Garrison \& von Ellenrieder, 2007: [LC]; JUJ, SAL (CHS; YUN) - L \{Not described\}.

Cyanallagma bonariense (Ris, 1913): [LC]; BUE, CBA, COR (Lozano et al., 2012), ENT, MIS

(4) (CHS; CYM; DIP; ESP; PAM) - L \{Bulla, 1973b\}.

Cyanallagma interruptum (Selys, 1876): [LC]; CHU, MEN, NEU, RIO, SCR (ALT; BPA; EPA;

MLM) — L \{Needham \& Bullock, 1943; Bulla, 1973b\}.

Cyanallagma nigrinuchale (Selys, 1876): [NA]; MIS (SEL) — L \{Not described\}.

Enallagma novaehispaniae Calvert, 1907: [LC]; JUJ, SAL, SGO, TUC (Rodríguez \& Molineri, 2013) (CHS; MSB; YUN) — L \{Novelo Gutiérrez, 2005\}.

Epipleoneura venezuelensis Rácenis, 1955: [NA]; ENT (Pessacq, 2014), SFE (3) (DIP) — L

\{Not described\}. The record from ENT published by Pessacq (2014) is the same as the record from Entre Ríos mentioned as Epipleoneura sp. in the checklist of Argentina (von Ellenrieder \& Muzón, 2008).

Homeoura chelifera (Selys, 1876): [NA]; BUE, CHA, COR, ENT, FOR, JUJ, MIS, SAL, SLU

(2), SFE, TUC (CHS; CYM; DIP; ESP; EST; MSB; PAM; SEL; YUN) — L \{Bulla, 1971a;

Lozano, Muzón, \& Torres, 2009; Needham, 1904\}.

Homeoura lindneri (Ris, 1928): [NA]; BUE, CHA, COR, ENT, FOR (Ris, 1928; von Ellenrieder, 2010), MIS (17, 18a, 18b), SFE (CYM; DIP; ESP; EST; SEL) — L \{Lozano et al., 2009\}. The first record from Formosa was by Ris (1928) as Acanthagrion lindneri. This information was not included in the last checklist (von Ellenrieder \& Muzón, 2008). von Ellenrieder (2010) mentioned this species again for Formosa.

Ischnura capreolus (Hagen, 1861): [NA]; BUE, COR, ENT, FOR (von Ellenrieder, 2010), JUJ, LAP (del Palacio et al., 2017), MIS, SAL, SFE, SGO (Navás, 1922), TUC (CHH; CHS; CYM; DIP; ESP; EST; PAM; SEL; YUN) — L \{Geijskes, 1941\}.

Ischnura fluviatilis Selys, 1876: [LC]; BUE, CAT, CHA, CHU (Muzón, Pessacq et al., 2014), CBA, COR, ENT, FOR, JUJ, LAP (del Palacio et al., 2017), LAR, MEN, MIS, NEU, RIO, SAL, SJU, SLU (3), SFE, SGO, TUC (ALT; CHH; CHS; CYM; DIP; EPA; ESP; 
EST; MLM; MSB; PAM; SEL; YUN) — L \{Rodrigues da Fonseca \& Pujol-Luz, 1999; von Ellenrieder \& Muzón, 2003b\}.

Ischnura ultima Ris, 1908: [NA]; BUE (Muzón, del Palacio, \& Ramos, 2014), CBA, JUJ, MEN, SAL, TUC (ALT; CHS; EPA; MLM; MSB; PAM; YUN) — L \{Muzón \& Pessacq, $2005\}$.

Leptagrion andromache Hagen in Selys, 1876: [NA]; MIS (Muzón, Weigel Muñoz, \& Campos, 2009) (SEL) — L \{Muzón et al., 2009; Costa, Oldrini, \& Anjos Santos, 2009\}.

Mecistogaster amalia (Burmeister, 1839): [DD]; MIS (SEL) — L \{Muzón, Weigel Muñoz, \& Campos, 2010\}.

Mecistogaster lucretia (Drury, 1773): [NA]; MIS (SEL) — L \{Not described\}. There are two subspecies described: M. lucretia hauxwelli and M. lucretia lucretia, in Argentina only $M$. lucretia lucretia has been recorded.

Mecistogaster ornata Rambur, 1842: [LC]; JUJ, SAL (YUN) — L \{Ramírez (1995) only M. ornata ornata\}. There are two subspecies described: $M$. ornata acutipennis and $M$. ornata ornata, in Argentina only M. ornata ornata has been recorded.

Metaleptobasis selysi Santos, 1956: [NA]; MIS (von Ellenrieder, 2013) (SEL) — L \{Not described\}.

Neoneura confundens Wasscher \& van't Bosch, 2013: [NA]; BUE (1), JUJ (Rodríguez et al., 2014), MIS (11), SAL (PAM; SEL; YUN) — L \{Not described\}.

Neoneura ethela Williamson, 1917: [NA]; COR, ENT, MIS (CYM; ESP; PAM; SEL) — L \{Souza, Pepinelli, \& Neiss, 2012\}.

Neoneura fulvicollis Selys, 1886: [LC]; MIS (SEL) — L \{De Marmels, 2007\}.

Neoneura sylvatica Hagen in Selys, 1886: [NA]; MIS (SEL) — L \{Not described $\}$.

Neoneura waltheri Selys, 1886: [NA]; MIS (SEL) — L \{Not described\}.

Oxyagrion ablutum (Calvert, 1909): [LC]; CAT, CBA, JUJ, LAR, MIS, SAL, SLU, TUC (CHS; MSB; YUN) — L \{Pessacq, Muzón, \& von Ellenrieder, 2005\}.

Oxyagrion basale Selys, 1876: [NA]; MIS (CYM) — L \{Bulla, 1973a; Costa, Souza, \& Santos, $2000\}$.

Oxyagrion brevistigma Selys, 1876: [NA]; MIS (SEL) — L \{Not described\}.

Oxyagrion bruchi Navás, 1924: [LC]; CBA, JUJ, SAL, TUC (ESP; YUN) — L \{von Ellenrieder \& Garrison, 2006\}.

Oxyagrion chapadense Costa, 1978: [NA]; BUE, CBA, COR, MIS (CHS; CYM; PAM; SEL) L \{Costa, Souza, et al., 2000\}.

Oxyagrion hempeli Calvert, 1909: [NA]; BUE, CBA, MIS (CHS; PAM; SEL) — L \{Bulla, $1973 \mathrm{a}\}$.

Oxyagrion rubidum (Rambur, 1842): [NA]; BUE, CHU, CBA, COR, ENT, JUJ, LAP (del Palacio et al., 2017), MEN, NEU, RIO, SAL, SLU (1), SFE, SGO, TUC (Rodríguez et al., 2018) (CHS; EPA; ESP; EST; MLM; PAM; YUN) — L \{Needham \& Bullock, 1943\}.

Oxyagrion terminale Selys, 1876: [NA]; BUE, COR, ENT, MIS, SFE (CYM; DIP; ESP; PAM; SEL) — L \{Bulla, 1973a\}.

Peristicta aeneoviridis Calvert, 1909: [NA]; COR, ENT, MIS (CYM; DIP; ESP; PAM; SEL) L \{Schröder, Anjos-Santos, Rippel, \& Pessacq, 2020\}.

Peristicta forceps Hagen in Selys, 1860: [NA]; BUE, COR, ENT, MIS (ESP; PAM; SEL) — L \{Pessacq, 2007\}.

Peristicta lizeria Navás, 1920: [DD]; BUE — L \{Not described\}. According to Pessacq (2008) the identity of this species is doubtful, because its type material is lost and its original description is poor. This species could be a synonym of one of the other species of Peristicta. In the original description there is no reference to the exact locality, Muzón et al. (2015) believe that this species was collected in DIP or near DIP because most localities from Buenos Aires province, cited in Navás papers, correspond to DIP or towns close to the Paraná Delta front. 
Protallagma titicacae (Calvert, 1909): [NA]; CAT (Rodríguez et al., 2018), JUJ, SAL (ALT; PUN) — L \{Bulla, 1972\}.

*Telagrion longum Selys, 1876: [NA]; MIS (5), SAL (3, 4) (SEL, YUN) — L \{Not described\}. Telebasis carmesina Calvert, 1909: [LC]; MIS, SAL (SEL; YUN) — L \{Not described\}.

Telebasis carminita Calvert, 1909: [NA]; SFE — L \{Not described\}.

Telebasis griffinii (Martin, 1896): [LC]; COR, ENT, JUJ, MIS, SAL (DIP; EST; SEL; YUN) L \{Guillermo-Ferreira \& Bispo, 2013\}.

Telebasis inalata (Calvert, 1961): [NA]; JUJ, SAL (Garrison, 2009; von Ellenrieder \& Garrison, 2007) (YUN) — L \{Not described\}.

Telebasis obsoleta (Selys, 1876): [LC as Helveciagrion obsoletum]; COR, FOR, MIS, SAL (von Ellenrieder, 2010) (CHH; CYM; ESP; EST; SEL) — L \{Lozano et al., 2012\}.

Telebasis simulacrum (Calvert, 1909): [LC as Helveciagrion simulacrum]; COR (EST) - L \{Not described\}.

Telebasis theodori (Navás, 1934): [NA]; MIS (SEL) — L \{Not described\}.

Telebasis willinki Fraser, 1948: [LC]; BUE, CHA, CBA (Zapata \& Pereyra, 2016), COR, ENT (1a, 1c, 2, 3, 6, 7, 8, 9), FOR, JUJ, SAL, SFE, TUC (CHH; CHS; CYM; DIP; ESP; EST; PAM; YUN) — L \{Bulla, 1970\}.

Tigriagrion aurantinigrum Calvert, 1909: [NA]; MIS, SAL (SEL; YUN) — L \{Not described\}.

\section{DICTERIADIDAE}

Heliocharis amazona Selys, 1853: [NA]; MIS, SFE (CHH; CYM; SEL) — L \{Geijskes, 1986; Santos \& Costa, 1988\}.

\section{LESTIDAE}

Archilestes exoletus (Hagen in Selys, 1862): [NA]; MIS (SEL) — L \{Dalzochio \& Rodrigues, $2011\}$.

Lestes auritus Hagen in Selys, 1862: [LC]; MIS (SEL) — L \{Not described\}.

Lestes bipupillatus Calvert, 1909: [NA]; CHA, MIS (DIP; SEL) — L \{Costa \& Carneiro, 1994\}. Lestes dichrostigma Calvert, 1909: [LC]; COR, JUJ, MIS, SAL, TUC (Rodríguez \& Molineri, 2013) (CHS; CYM; SEL; YUN) — L \{Muzón \& Weigel Muñoz, 2007\}.

Lestes forficula Rambur, 1842: [LC]; COR (7), JUJ, MIS, SAL, SFE, TUC (Rodríguez et al., 2018) (CHS; CYM; SEL; YUN) — L \{Calvert, 1928; Klots, 1932\}.

*Lestes minutus Selys, 1862: [NA]; COR (4) (EST) — L \{Not described $\}$.

Lestes paulistus Calvert, 1909: [LC]; COR, MIS (ESP; SEL) — L \{Not described\}.

Lestes pictus Hagen in Selys, 1862: [NA]; COR, JUJ, MIS, SAL, TUC (Rodríguez \& Molineri, 2013) (CYM; SEL; YUN) — L \{Costa \& Carneiro, 1994; Santos, 1972a\}.

Lestes spatula Fraser, 1946: [NA]; BUE, CAT, CHA, COR, ENT, FOR (von Ellenrieder, 2010), LAP (1), MIS, SAL, SFE (Fraser, 1948), SGO, TUC (Rodríguez \& Molineri, 2013) (CHH; CHS; CYM; DIP; ESP; EST; PAM; SEL; YUN) - L \{Muzón, 1993\}.

Lestes tricolor Erichson, 1848: [NA]; MIS (SEL) — L \{Costa \& Carneiro, 1994\}.

Lestes undulatus Say, 1840: [LC]; BUE, CBA (1), ENT, LAP (del Palacio et al., 2017), MEN, MIS (6, 19), RIO, SFE (Navás, 1920) (BPA; CHS; DIP; EPA; ESP; MLM; PAM; SEL) — L \{Muzón, 1997\}. The record from Santa Fe was provided by Navás (1920). However, no mention of this species was done by von Ellenrieder and Muzón (2008).

\section{MEGAPODAGRIONIDAE}

Allopodagrion brachyurum De Marmels, 2001: [NA]; MIS (SEL) — L \{Neiss, Fiorentin, \& De Marmels, 2011\}.

Allopodagrion contortum (Hagen in Selys, 1862): [LC]; MIS (SEL) — L \{Not described\}. 
Table 1. Species added since von Ellenrieder and Muzón (2008).

\begin{tabular}{lll}
\hline Family & Species & Type of record \\
\hline Aeshnidae & Gynacantha mexicana & Rodríguez et al. (2018) \\
Aeshnidae & Triacanthagyna caribbea & Rodríguez et al. (2018) \\
Austropetaliidae & Hypopetalia pestilens & Pessacq and Brand (2009) \\
Austropetaliidae & Phyllopetalia apollo & Pessacq and Brand (2009) \\
Coenagrionidae & Acanthagrion ascendens & Lozano (2013) \\
Coenagrionidae & Acerathobasis nathaliae & New record \\
Coenagrionidae & Aeolagrion phillipi & von Ellenrieder (2010) \\
Coenagrionidae & Argia tamoyo & New record \\
Coenagrionidae & Epipleoneura venezuelensis & Pessacq (2014) \\
Coenagrionidae & Leptagrion andromache & Muzón et al. (2009) \\
Coenagrionidae & Metaleptobasis selysi & von Ellenrieder (2013) \\
Coenagrionidae & Telagrion longum & New record \\
Gomphidae & Archaeogomphus infans & New record \\
Gomphidae & Phyllocycla foliata & Belle (1988) \\
Gomphidae & Progomphus costalis & New record \\
Lestidae & Lestes minutus & New record \\
Libellulidae & Dasythemis esmeralda & New record \\
Libellulidae & Erythemis carmelita & von Ellenrieder (2010) \\
Libellulidae & Erythrodiplax amazonica & New record \\
Libellulidae & Erythrodiplax pallida & del Palacio and Muzón (2016) \\
Libellulidae & Erythrodiplax unimaculata & New record \\
Libellulidae & Micrathyria iheringi & New record \\
Libellulidae & Micrathyria stawiarskii & New record \\
Libellulidae & Nephepeltia berlai & von Ellenrieder (2014) \\
Libellulidae & Nephepeltia leonardina & Rodríguez et al. (2014) \\
Libellulidae & Oligoclada rubribasalis & von Ellenrieder and Garrison (2008a) \\
Libellulidae & Orthemis phillipi & von Ellenrieder (2010) \\
\hline & &
\end{tabular}

Allopodagrion erinys (Ris, 1913): [DD]; MIS — L \{Not described\}. Record for Misiones in Ris (1913) but exact locality unknown.

Heteragrion aurantiacum Selys, 1862: [LC]; MIS (CYM; SEL) — L \{Santos, 1968d\}.

Heteragrion triangulare Hagen in Selys, 1862: [DD]; MIS (SEL) — L \{Not described\}.

Teinopodagrion meridionale De Marmels, 2001: [LC]; CAT, JUJ, SAL, TUC (YUN) — L \{von Ellenrieder, 2006\}.

\section{POLYTHORIDAE}

Chalcopteryx rutilans (Rambur, 1842): [LC]; MIS (SEL) — L \{Santos \& Costa, 1987\}.

\section{Changes made since the checklist of von Ellenrieder and Muzón (2008)}

Since von Ellenrieder and Muzón (2008), one family (Synthemistidae) and 27 species have been added: two Aeshnidae, two Austropetaliidae, eight Coenagrionidae, three Gomphidae, one Lestidae, and 11 Libellulidae. From these, 10 are new records provided in this contribution (see comments below on each of these species), 15 species were recorded after the checklist of 2008, and two species were recorded before 2008 but omitted in that checklist (Table 1). In addition, eight species have changed their taxonomic status since the last checklist (Table 2). The family Synthemistidae is recorded for the first time in Argentina, due to the placement of Gomphomacromia within it in recent molecular studies (Carle et al., 2015).

Among the newly recorded species, Acerathobasis nathaliae was previously known from the Atlantic Forest of Brazil (von Ellenrieder \& Garrison, 2008b). This species is not common; in fact, A. nathaliae was previously known only from its type locality: São Pablo, Jacarei, Fazenda 
Table 2. Species that changed taxonomic status since von Ellenrieder and Muzón (2008).

\begin{tabular}{lll}
\hline Name in checklist 2008 & Current name & Reference \\
\hline Acanthagrion ablutum & Oxyagrion ablutum & von Ellenrieder and Lozano (2008) \\
Acanthagrion peruvianum & Acanthagrion floridense & Lozano et al. (2017) \\
Dythemis multipunctata & Dythemis nigra & Meurgey and Poiron (2011) \\
Helveciagrion obsoletum & Telebasis obsoleta & Garrison (2009) \\
Helveciagrion simulacrum & Telebasis simulacrum & Garrison (2009) \\
Homeoura ambigua & Argentagrion ambiguum & von Ellenrieder (2008) \\
Neoneura bilinearis & Neoneura confundens & Wasscher and Van 'T Bosch (2013) \\
Telebasis limoncocha & Telebasis griffini & Garrison (2009) \\
\hline
\end{tabular}

Santana do Rio Abaixo (Lencioni, 2004). Argia tamoyo is not a common species either, but it had a wider distribution range; it was previously known from Brazil and Bolivia (Lencioni, 2017).

Progomphus costalis has a wide distribution, where it has been recorded from Rio de Janeiro, Brazil, south to Uruguay (von Ellenrieder, 2009; von Ellenrieder, Molineri, \& Emmerich, 2009).

Lestes minutus is a rare South American species; there are scattered records in Colombia, Suriname, Trinidad, Venezuela and Brazil (Bota Sierra, 2014; Costa, Souza, et al., 2000; Lencioni, 2017).

The libellulids Dasythemis esmeralda, Erythrodiplax amazonica, E. unimaculata, and Micrathyria iheringi are widely distributed in South America. The records herein provided expand the southernmost limit of their distribution range. Micrathyria stawiarskii has a more restricted range, previously known from Goias to Rio Grande do Sul states in Brazil.

Phyllocycla foliata was described by Belle (1988) from specimens collected by Jurzitza in Misiones (Parque Nacional Iguazú). However, this species was not listed by von Ellenrieder \& Muzón (2008). Belle considered it to be very similar to $P$. argentina, which is sympatric with $P$. foliata. Therefore, it is important to revise the taxonomic status of this species.

Two species were removed from the checklist by von Ellenrieder and Muzón (2008): Aeolagrion inca and Micrathyria debilis. The former one was recorded for FOR based on a specimen collected in Estancia Guaycolec. This specimen belongs to A. phillipi. However, the identification was done prior to the description of A. phillipi (Tennessen, 2009). Micrathyria debilis is a common species in Central America. von Ellenrieder and Muzón (2008) mentioned this species for Entre Rios and Santa Fe based on records provided by Fraser (1947). However, it has not been found again in these provinces and is likely to be a misidentification.

\section{Endemic species of Argentina}

There are 17 species endemic to Argentina (Table 3; Figure 3). Even though Patagonia has remarkable endemics at the regional level (e.g. Neopetalidae), there are no Patagonian endemics exclusive of Argentina, all having been also recorded in Chile (Muzón, Pessacq et al., 2014).

Allopodagrion erinys, Peristicta lizeria, Phyllocycla foliata and Progomphus australis are only known from their original description; little is known on their biology and habitat preferences.

Castoraeschna decurvata inhabits lotic environments with small waterfalls and sand bottoms. Adults are fast flyers and were seen patrolling at $1.5 \mathrm{~m}$ above the river. Final-stage larvae were collected in vegetated areas near the shores (Rodrigues Capitulo \& Jurzitza, 1989). There are two disjunct areas where it has been recorded: one in Córdoba province and the other one in Entre Rios province; both are within the ESP ecoregion. Further research is needed in order to 
Table 3. Endemic species of Argentina.

\begin{tabular}{ll}
\hline Familiy & Species \\
\hline Aeshnidae & Castoraeschna decurvata \\
Aeshnidae & Rhionaeschna haarupi \\
Aeshnidae & Rhionaeschna pallipes \\
Aeshnidae & Staurophlebia bosqi \\
Coenagrionidae & Andinagrion garrisoni \\
Coenagrionidae & Andinagrion peterseni \\
Coenagrionidae & Argia jujuya \\
Coenagrionidae & Peristicta lizeria \\
Gomphidae & Phyllocycla foliata \\
Gomphidae & Phyllocycla vesta \\
Gomphidae & Phyllogomphoides joaquini \\
Gomphidae & Progomphus auropictus \\
Gomphidae & Progomphus australis \\
Libellulidae & Oligoclada haywardi \\
Libellulidae & Oligoclada rubribasalis \\
Megapodagrionidae & Allopodagrion erinys \\
Synthemistidae & Gomphomacromia nodisticta \\
\hline
\end{tabular}

determine if this species has a disjunct distribution within Argentina or if it is present within intermediate areas.

Rhionaeschna haarupi occurs in marshy areas of Andean streams and rivers of the Precordillera (mountain ranges lying before the Andes) where it has been found at elevations between 900 and $2015 \mathrm{~m}$. This species is very rarely seen in the field.

Rhionaeschna pallipes has a unique distribution among the species traditionally included within the former subgenus Marmaraeschna, as it is not restricted to the Andes (Muzón \& von Ellenrieder, 2001); it has also been recorded in hilly areas of Córdoba and Buenos Aires. There are two old records in Santa Fe province in the Paraná river basin, but as there are no mountains there, Muzón and von Ellenrieder (2001) believe these records are questionable. This species inhabits rapidly moving waters. Larvae were found under stones and clinging to submerged roots of marginal vegetation in creeks (von Ellenrieder \& Muzón, 2003a).

Members of the genus Staurophlebia are among the largest species of Argentine dragonflies (total length $80-95 \mathrm{~mm}$ ). Staurophlebia bosqi is a rare species that has been recorded in a small area of the lower delta of the Paraná river. It inhabits streams within thick marginal forests of flood plains. It is very similar to Staurophlebia reticulata, the other congener recorded in Argentina; a taxonomic revision of these two is necessary in order to test their specific status.

The genus Andinagrion is almost exclusively distributed in Argentina; except for A. saliceti which has been also recorded in Uruguay, the other two known species of the genus, A. garrisoni and A. peterseni, are endemic to the country. Andinagrion garrisoni is known to occur in the Yungas (von Ellenrieder, 2009), whereas A. peterseni is more widely distributed within Argentina, found from Yungas south to Patagonia and east to Buenos Aires. These species inhabit lentic environments at stream edges in hilly areas with abundant aquatic vegetation.

Argia jujuya is also endemic to the Argentine Yungas; among the four species of Argia reported in the region (NW Argentina), A. jujuya occupies the smallest range and is not as frequent and abundant as other species (Molineri \& Rodríguez, 2013). It inhabits lotic environments strongly vegetated with rooted aquatic and semiaquatic plants (Molineri \& Rodríguez, 2013).

There are two endemic gomphids recorded from Buenos Aires: Phyllocycla vesta and Phyllogomphoides joaquini. Both species are rarely seen in the field or in collections. Adults are very good flyers that inhabit lotic environments. Only the larva of Phyllogomphoides joaquini has been described, and as other gomphids it was found burrowed in sandy bottoms. 

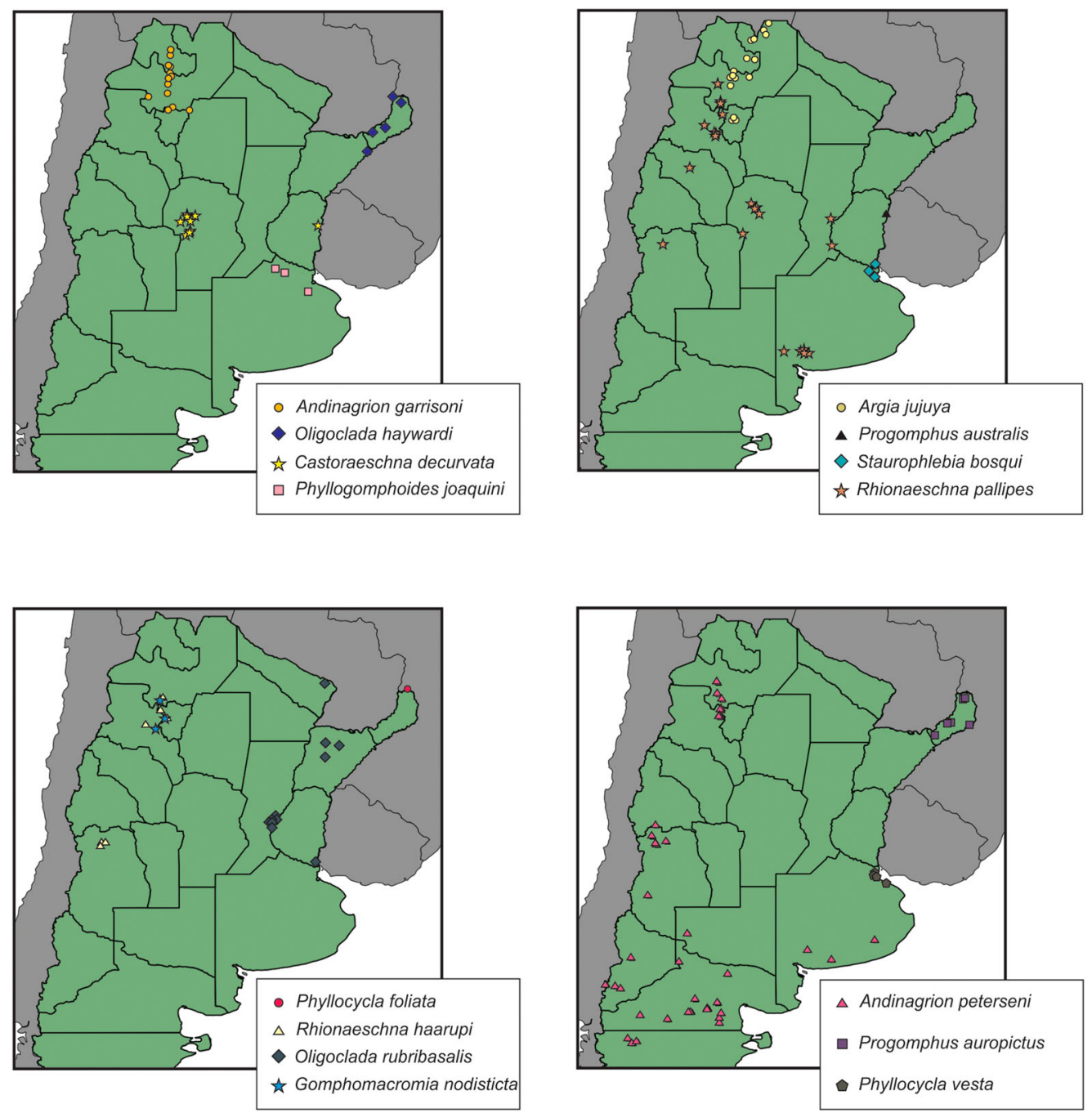

Figure 3. Maps showing distribution of endemic species.

Progomphus auropictus occurs in the Paranaense Forest in Misiones (von Ellenrieder \& Muzón, 2008). Adults were seen flying in gallery forest streams with rocky substrate. Little is known on the biology of this species; the larva is unknown.

Gomphomacromia nodisticta is a rare species endemic to the ecotone between the Monte de Sierras y Bolsones and Yungas ecoregions of the Brown and Pacheco (2006) scheme. It has been recorded at elevations between 2400 and $2700 \mathrm{~m}$. This species inhabits mountain streams; the larva is still unknown.

Oligoclada haywardi was described by Fraser (1947) from Misiones. It was not found again until 2009, when it was discovered in Corrientes; in 2011 and 2012 it was rediscovered in Misiones. Adults were collected while flying in gallery forest streams and in lentic environments near these streams. The larva is unknown. It is likely that this species is also present in Brazil.

Finally, Oligoclada rubribasalis can be found at shallow marshes surrounding ponds and rivers, perching on leaves exposed to the sun in northeastern Argentina. Reproductive behavior was not observed, and the larva is still unknown (von Ellenrieder \& Garrison, 2008a). 


\section{Provincial records}

Since the publication of the checklist in 2008, a total of 237 provincial records have been added (Figure 4); of these, 150 were either published after the publication of the checklist of von Ellenrieder and Muzón (2008) or were published before and omitted in it (Table 4), and 87 are new provincial records added in this contribution.

Formosa, Salta, Tucumán and La Pampa are the provinces with more new records published since 2008; most of the records can be found in papers dealing with regional diversity (del Palacio et al., 2017; Rodríguez et al., 2018; Rodríguez \& Molineri, 2013; von Ellenrieder, 2010; von Ellenrieder \& Garrison, 2007). Most of the new records added here are for Misiones and Corrientes (17 and 16 respectively); these are highly diverse provinces and have been intensively surveyed during the last decade. Santa Fe and San Luis follow with 10 new records each; in this case these provinces had been poorly studied and with few field samples in the area many novelties were found.

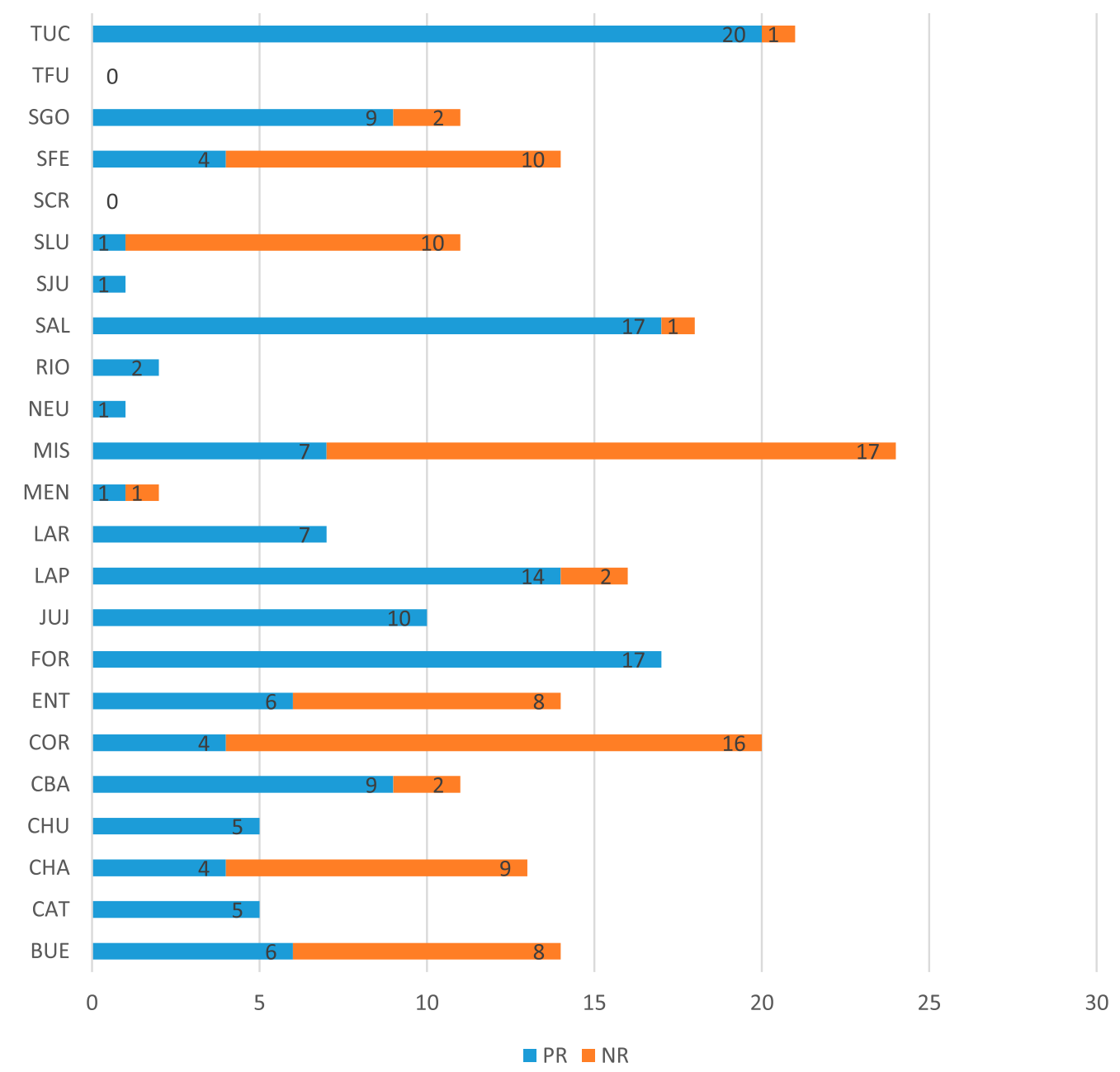

Figure 4. Number of new records per province. PR: Published records not included in Checklist 2008. NR. New records in this contribution. 
Table 4. Records previous to von Ellenrieder and Muzón (2008) but omitted in it.

\begin{tabular}{|c|c|c|c|}
\hline Species & Prov & Record & Named as \\
\hline Argentagrion ambiguum & CAT & Ris (1918) & Acanthagrion ambiguum \\
\hline Homeoura lindneri & FOR & Ris (1928) & Acanthagrion lindneri \\
\hline Ischnura capreolus & SGO & Navás (1922) & Ceratura capreola \\
\hline Telebasis inalata & SAL & $\begin{array}{l}\text { von Ellenrieder and } \\
\text { Garrison (2007) }\end{array}$ & Telebasis inalata \\
\hline Lestes undulatus & SFE & Navás (1920) & Lestes undulatus \\
\hline Diastatops intensa & FOR & Ris (1928) & Diastatops pullata \\
\hline Erythemis vesiculosa & CAT & Ris (1928) & Lepthemis vesieulosa \\
\hline Erythrodiplax atroterminata & ENT & $\begin{array}{l}\text { Rodrigues Capitulo } \\
\text { and Muzón (1985) }\end{array}$ & Erythrodiplax atroterminata \\
\hline Erythrodiplax corallina & SFE & Förster (1914) & $\begin{array}{l}\text { Erythrodiplax nutrina. Borror } \\
\text { (1942) synonymized this } \\
\text { with } E \text {. corallina }\end{array}$ \\
\hline Erythrodiplax fusca & SAL & Ris (1918) & Erythrodiplax connata fusca \\
\hline Erythrodiplax fusca & TUC & Ris (1918) & Erythrodiplax connata fusca \\
\hline Erythrodiplax ochracea & MEN & Navás (1924) & Erytrodiplax schracea \\
\hline Erythrodiplax ochracea & SGO & Navás (1922) & Erythrodiplax ochracea \\
\hline Erythrodiplax umbrata & SGO & Navás (1922) & Erythrodiplax umbrata \\
\hline Miathyria marcella & CBA & Navás (1922) & Miathyria marcella \\
\hline Micrathyria hypodidyma & SFE & Navás (1917) & $\begin{array}{l}\text { Micrathyria didyma var. } \\
\text { hypodidyma }\end{array}$ \\
\hline Orthemis discolor & CBA & Navás (1922) & Orthemis ferruginea \\
\hline Pantala flavescens & $\mathrm{CBA}$ & Navás (1922) & Pantala flavescens \\
\hline Tauriphila argo & BUE & Navás (1930) & Tauriphila argo \\
\hline Tramea binotata & SFE & Navás (1930) & Tramea binotata \\
\hline Tramea binotata & SGO & Navás (1922) & Tramea binotata \\
\hline
\end{tabular}

There are 80 provincial records belonging to 62 species that could not be georeferenced (Figure 5). The reason for this is that either the localities mentioned in the publication were not found or only provincial records were provided.

Seven species cited for Argentina are known only by provincial records: Telebasis carminita for Santa Fe, Archaeogomphus densus, Erythrodiplax anomala, Erythrodiplax famula, Erythrodiplax lativittata, and Allopodagrion erinys for Misiones and Macrothemis tessellata for Buenos Aires and Misiones. Further research is needed in order to confirm the presence of these species within the provinces in which they were recorded.

\section{Provincial records removed}

Aphylla dentata: Removed from BUE, ENT and SFE.

von Ellenrieder and Muzón (2008) list this species for Buenos Aires, Entre Ríos, and Santa Fe. The records from Buenos Aires and Santa Fe belong to Ris (1904) and Navás (1927a, 1928, 1930); the record from Entre Ríos could not be found, but there is a mention of Aphylla dentata from Delta del Paraná (Rodrigues Capitulo, Mola, \& Agopian, 1991). Belle (1992) stated that all specimens cited by Navás as A. dentata probably belong to Aphylla distinguenda. Therefore, taking into account that $A$. dentata has not been found again in these provinces we remove it from Buenos Aires, Entre Ríos and Santa Fe and transfer these records to A. distinguenda.

Erythrodiplax anomala: Removed from BUE.

This species is distributed in eastern Brazil south to northeastern Argentina (Misiones). Navás (1922) mentioned this species for BUE. Santos (1953) says that its general appearance is similar to E. media and E. paraguayensis. Therefore, this species is likely to be a misidentification. 


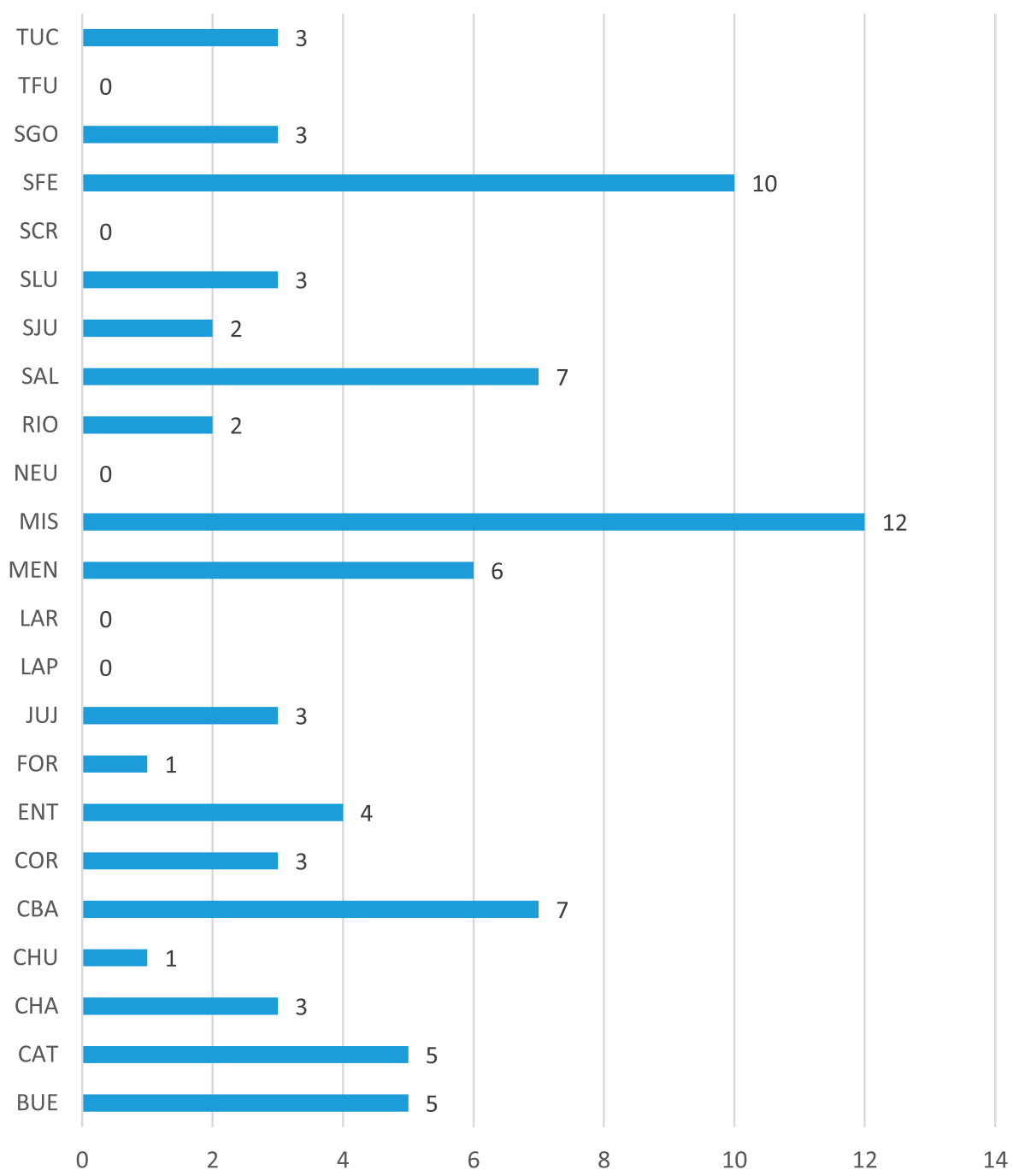

Figure 5. Number of species per province only known by provincial records.

\section{Erythrodiplax juliana: Removed from ENT}

This species has been removed from ENT since the specimens belong to E. chromoptera.

\section{Knowledge of larvae}

The knowledge of the larvae of the Odonata recorded in Argentina is still far from complete. Of the 282 species recorded, the larva of only $184(65 \%)$ have been described (Figure 6). The situation at the generic level is much better, as of the 85 genera recorded, $70(82 \%)$ have at least one Argentinean species with its larva described, and of the 15 remaining genera the larva of seven of them is known based on species not recorded in Argentina.

Since the last published checklist (von Ellenrieder \& Muzón, 2008), the larvae of 35 species have been described, mostly Libellulidae and Coenagrionidae with 15 and 13, respectively (Table 5). 


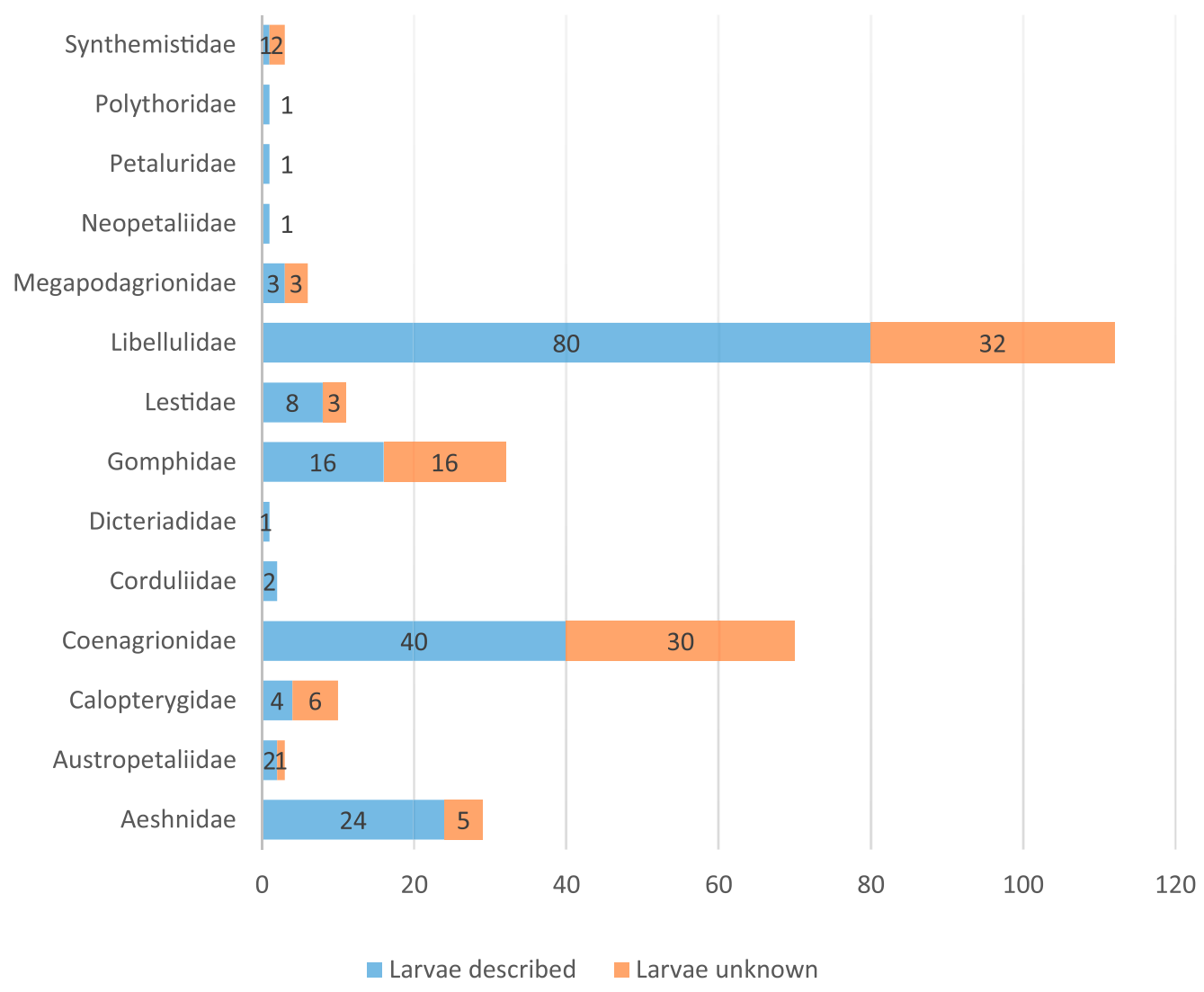

Figure 6. Larvae described and unknown for each family.

\section{Ecoregional knowledge}

At the ecoregional level, the Selva Paranaense and Yungas are the richest (Figures 7, 8A). This is not surprising, taking into account that many of the neotropical species have their southernmost distribution limits within these ecoregions. When plotting the richness of the ecoregions (Figure 8A, B), it becomes evident that the knowledge of $\mathrm{CHH}$ is still far from complete; fewer species are recorded than in neighboring ecoregions (Figure 8B). Little is known about the odonates of the Puna, where only three species have been recorded, and it is likely that this number is higher. These regions need to be better surveyed in order to improve their state of knowledge.

\section{Conservation status}

The Odonata is the first insect order to be assessed on a representative global scale for the IUCN Red List of Threatened Species (Clausnitzer et al., 2009). From the 282 species recorded in Argentina, only 113 species $(40.07 \%)$ have been assessed globally using the IUCN categories and criteria (Figure 9). However, most of these assessments (73.45\%) were done before 2010 and need to be updated (Figure 10).

Efforts are being made to complete the global assessment of all neotropical species and publish them in the Red List. Therefore, it is estimated that all the Latin American species are going to 
Table 5. Larvae described since von Ellenrieder and Muzón (2008).

\begin{tabular}{|c|c|c|}
\hline Familiy & Species & Larval description \\
\hline Aeshnidae & Limnetron antarcticum & del Palacio and Muzón (2014) \\
\hline Aeshnidae & Rhionaeschna vigintipunctata & Rodríguez and Molineri (2014) \\
\hline Calopterygidae & Mnesarete pudica & Guillermo-Ferreira and Bispo (2012) \\
\hline Coenagrionidae & Acanthagrion floridense & Lozano et al. (2017) \\
\hline Coenagrionidae & Acanthagrion gracile & Anjos Santos et al. (2011) \\
\hline Coenagrionidae & Acanthagrion lancea & Anjos Santos et al. (2011) \\
\hline Coenagrionidae & Argia croceipennis & Costa et al. (2008) \\
\hline Coenagrionidae & Argia jujuya & Molineri and Rodríguez (2013) \\
\hline Coenagrionidae & Argia serva & del Palacio et al. (2018) \\
\hline Coenagrionidae & Homeoura lindneri & Lozano et al. (2009) \\
\hline Coenagrionidae & Mecistogaster amalia & Muzón, Weigel Muñoz, and Campos (2010) \\
\hline Coenagrionidae & Neoneura ethela & Souza et al. (2012) \\
\hline Coenagrionidae & Neoneura fulvicollis & De Marmels (2007) \\
\hline Coenagrionidae & Peristicta aeneoviridis & Schröder et al. (2020) \\
\hline Coenagrionidae & Telebasis griffinii & Guillermo-Ferreira and Bispo (2013) \\
\hline Coenagrionidae & Telebasis obsoleta & Lozano et al. (2012) \\
\hline Gomphidae & Progomphus joergenseni & Muzón and Lozano (2011) \\
\hline Lestidae & Archilestes exoletus & Dalzochio and Rodrigues (2011) \\
\hline Libellulidae & Diastatops intensa & Costa et al. (1999) \\
\hline Libellulidae & Erythrodiplax atroterminata & Garré et al. (2008) \\
\hline Libellulidae & Erythrodiplax connata & Lozano et al. (2011) \\
\hline Libellulidae & Erythrodiplax corallina & Garré et al. (2008) \\
\hline Libellulidae & Erythrodiplax media & Dalzochio et al. (2018) \\
\hline Libellulidae & Idiataphe longipes & Oldrini et al. (2005) \\
\hline Libellulidae & Macrothemis declivata & Salgado et al. (2013) \\
\hline Libellulidae & Macrothemis hemichlora & Salgado et al. (2013) \\
\hline Libellulidae & Macrothemis heteronycha & Costa et al. (2010) \\
\hline Libellulidae & Macrothemis imitans & Salgado et al. (2013) \\
\hline Libellulidae & Macrothemis tenuis & Salgado et al. (2013) \\
\hline Libellulidae & Micrathyria pseudeximia & Dalzochio (2009a) \\
\hline Libellulidae & Nephepeltia berlai & Dalzochio (2009b) \\
\hline Libellulidae & Orthemis cultriformis & Carvalho and Werneck-de-Carvalho (2005) \\
\hline Libellulidae & Perithemis lais & Costa and Régis (2005) \\
\hline Megapodagrionidae & Allopodagrion brachyurum & Neiss et al. (2011) \\
\hline Polythoridae & Chalcopteryx rutilans & Santos and Costa (1987) \\
\hline
\end{tabular}

be assessed by 2021. At present, progress is being made in completing the National Red List of Odonata, the first national red list of an insect order within Argentina.

\section{Species yet to be described or in manuscript}

Limnetron n. sp.: According to von Ellenrieder \& Garrison (2007) Limnetron n. sp. was mentioned as L. debile by von Ellenrieder (2001c).

Gen nov. sp. nov. (Coenagrionidae) Muzón \& Lozano (in press): This species has been described based on specimens collected in COR (CYM) and Brazil. Specimens from Argentina were collected in a marshy area around a pine plantation was crossed by a small creek.

Angelagrion sp.: Specimens from this genus were collected in ENT (DIP) and are under study.

\section{Argentina in a South American context}

The knowledge of the Odonata from South America has grown steadily in recent years, with many South American entomologists working on ecology, diversity, taxonomy, and conservation of dragonflies and damselflies. However, there are still gaps that need to be addressed: there are 


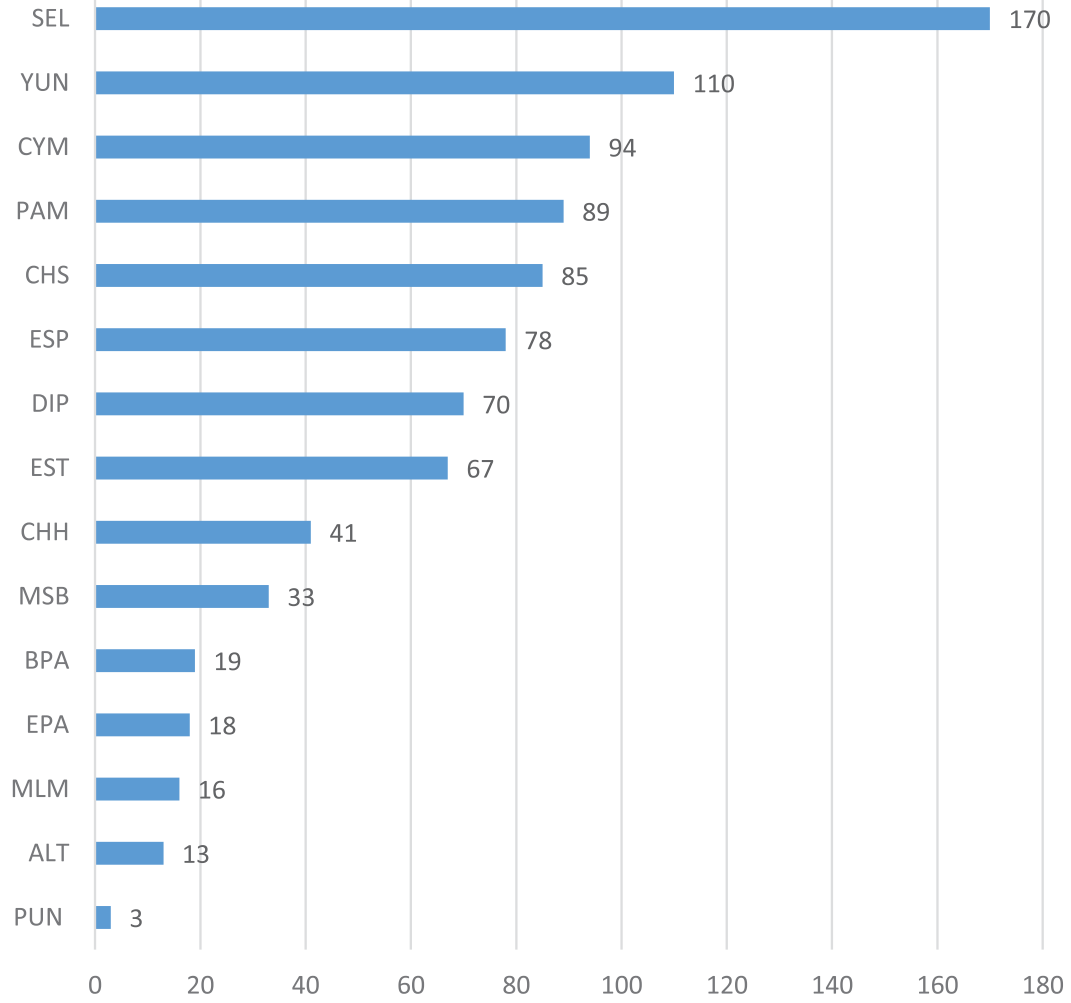

Figure 7. Number of species recorded for each ecoregion.

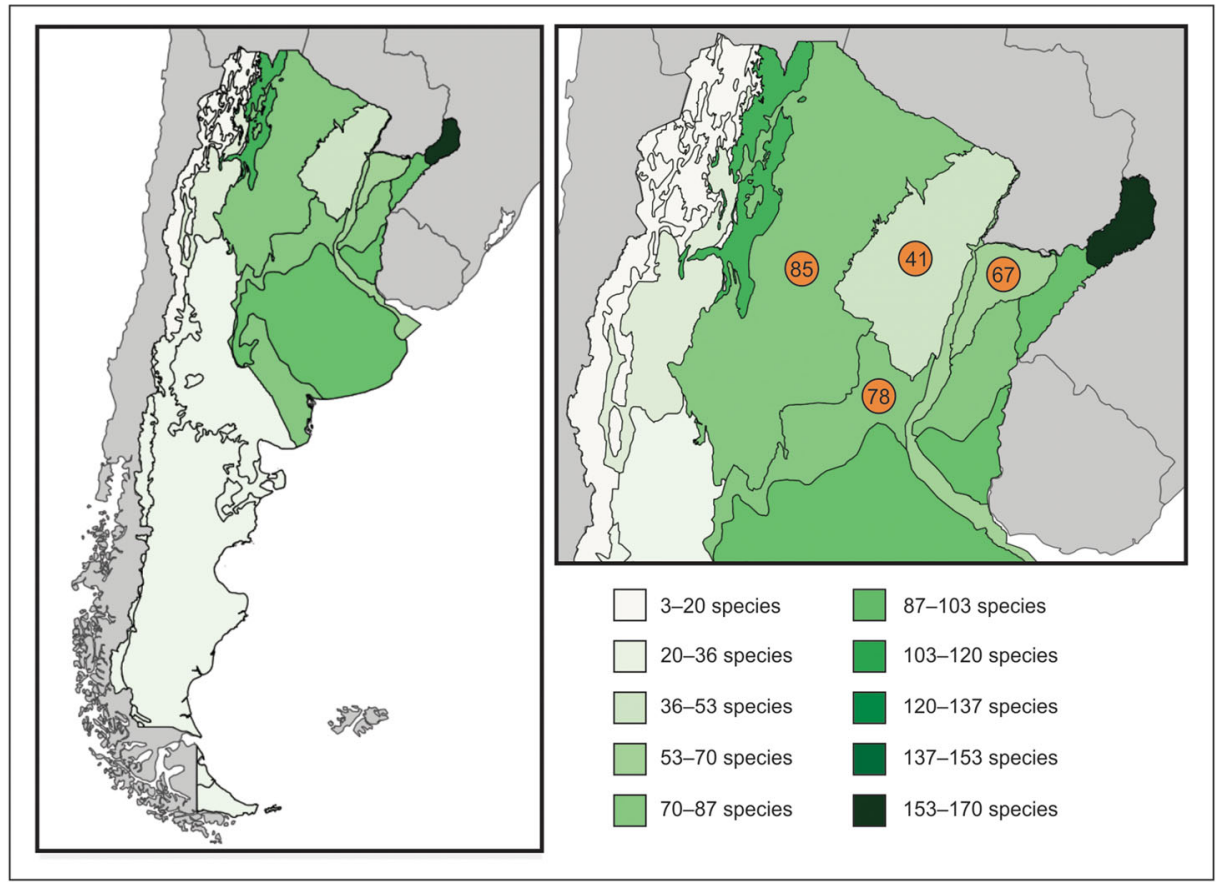

Figure 8. Map showing diversity per ecoregion. Colors correlate with alpha diversity, the darker the more diverse. (A) Argentina; (B) detail of Chaco Húmedo showing that the number of species recorded is less than neighboring ecoregions. 

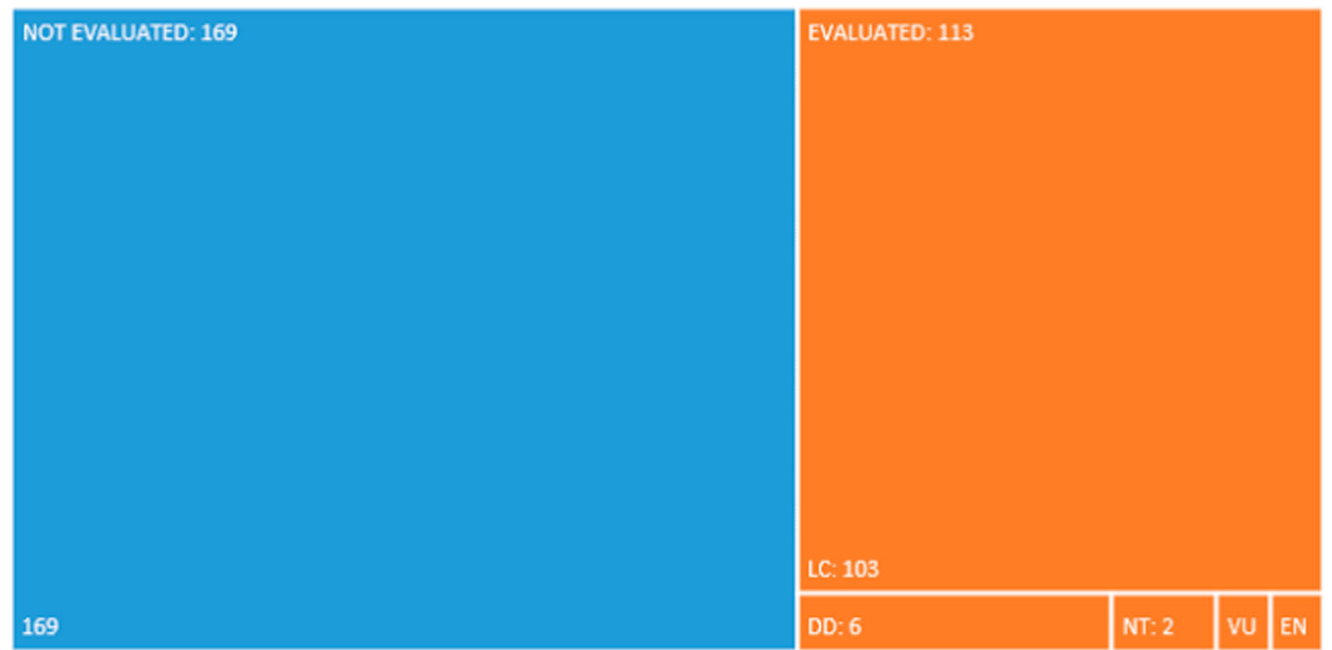

Figure 9. Proportion of species assessed according to the IUCN categories and criteria.

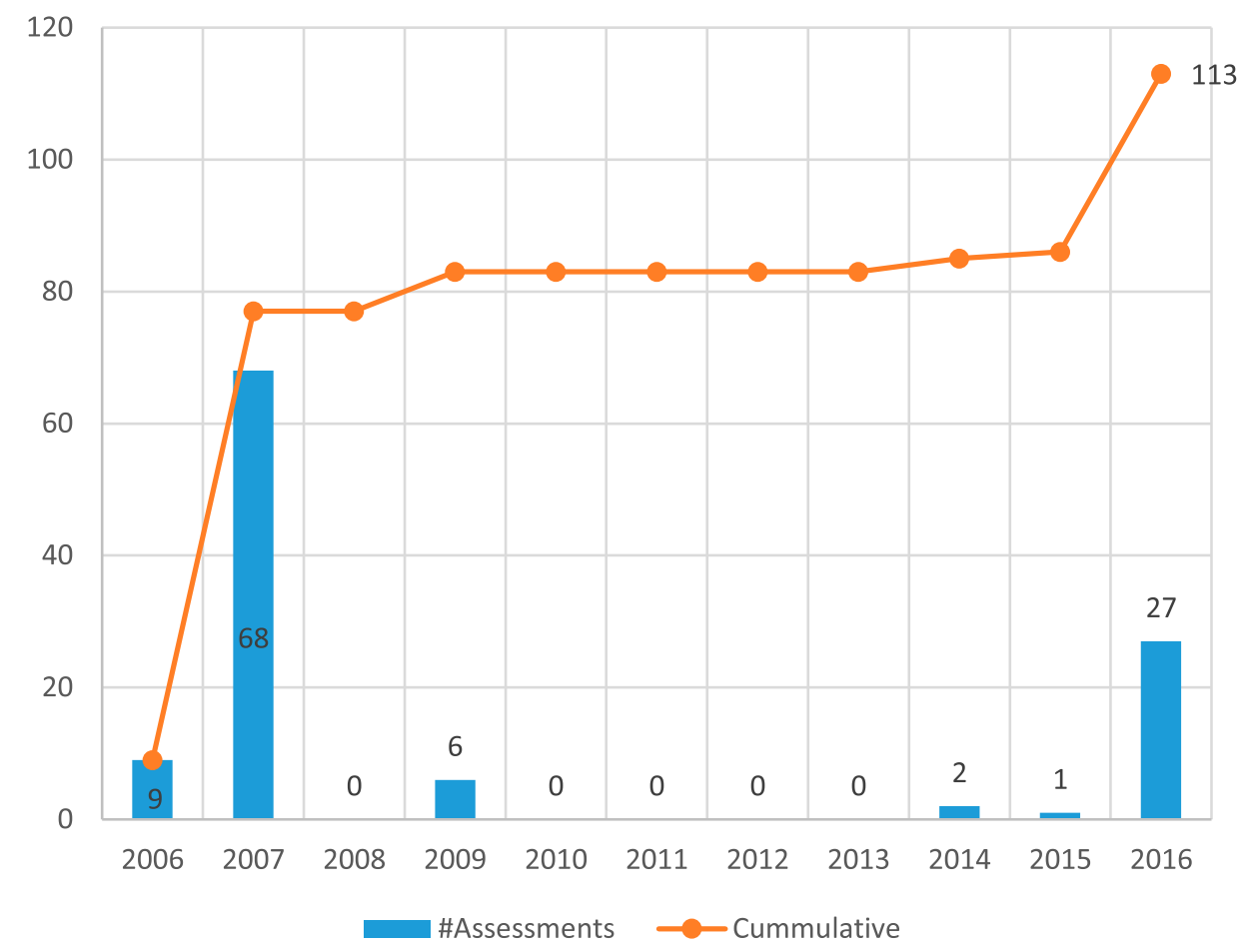

Figure 10. Number of Red List Assessments done per year.

many countries that do not have updated checklists (Table 6), and the specific richness is probably underestimated (for example in Bolivia and Paraguay); this can be evidenced in the fact that many new species are being described every year. Besides, little is known on the ecology, habitat preferences, and larvae of many species.

When comparing the specific richness between South American countries, Argentina, with 282 known species, is behind Brazil, Venezuela, Peru, Ecuador, and Colombia (Table 6). This 
Table 6. Species richness, endemics and information of last checklist available for South American countries.

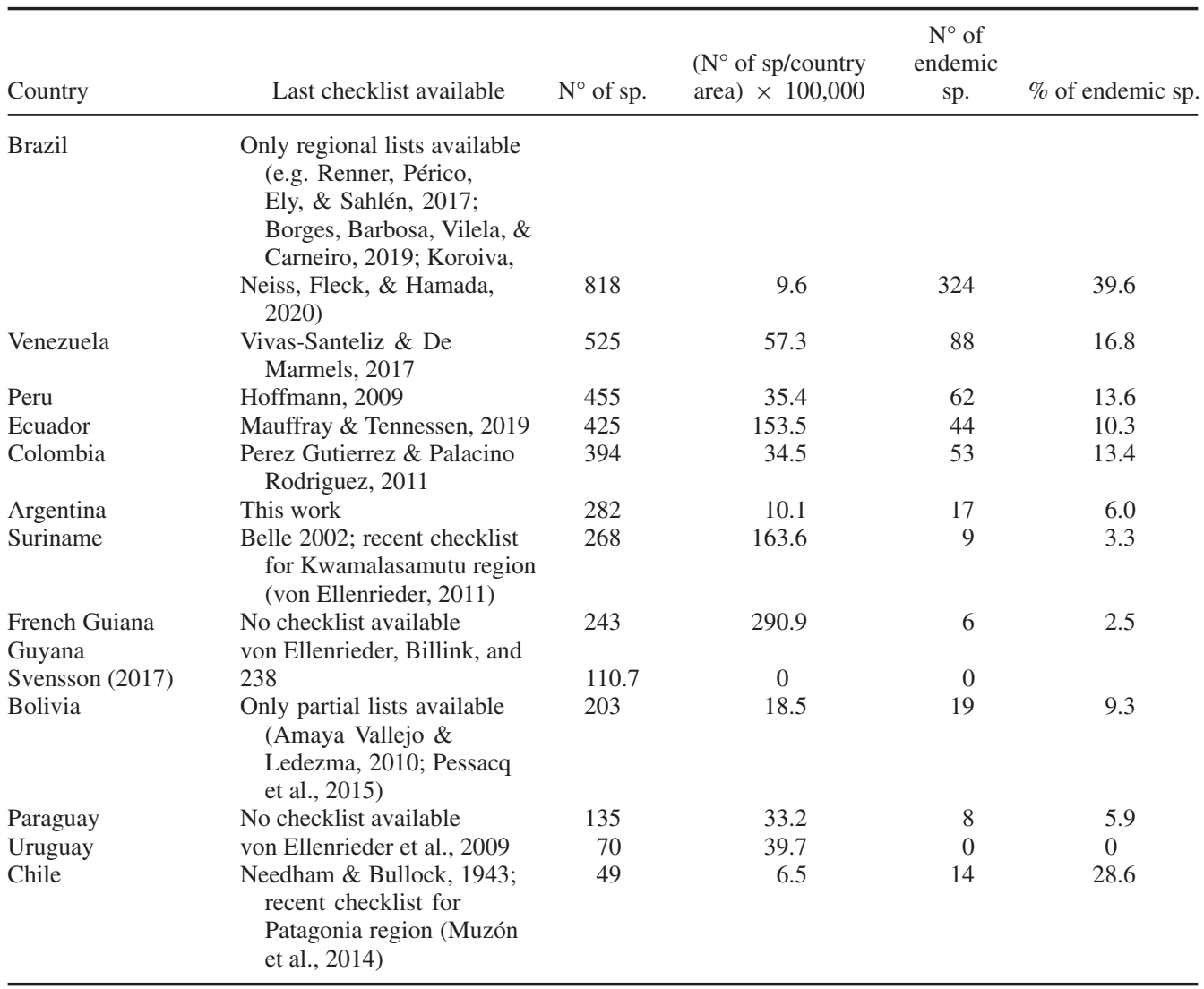

is not surprising taking into account that most of these countries are known for their high specific richness. However, when weighting the species richness with the country area, the situation is very different, with Argentina being one of the countries with the lowest values of species richness (Table 6).

The number of endemic species varies greatly among the South American countries. Brazil, with more than 300 species, is the country with the greatest number of endemic species. It is followed by Venezuela, Peru, Ecuador, and Colombia, as with the number of known species. However, when the percentage of endemic species is considered, Chile with $28.6 \%$ of endemic species is second to Brazil (Table 6).

\section{Acknowledgements}

The authors would like to thank Natalia von Ellenrieder for her helpful advice and for providing valuable records used in this contribution.

\section{ORCID}




\section{References}

Amaya Vallejo, V., \& Ledezma, J. (2010). Libélulas (Odonata: Anisoptera) de la colección Entomológica del Museo de Historia Natural Noel Kempff Mercado, Santa Cruz de la Sierra, Bolivia. Kempffiana, 6(2), 40-47. Retrieved from http://museonoelkempff.org/museo/kempffiana/

Anjos Santos, D., Carriço, C., Costa, J. M., \& Santos, C. T. (2011). Description of the final instar larvae of Acanthagrion gracile (Rambur) and Acanthagrion lancea Selys (Odonata: Coenagrionidae). Zootaxa, 2832, 44-50. doi:10.11646/zootaxa.2832.1.2

Bachmann, A. O. (1963). La ninfa de Staurophlebia bosqi Navás, 1927 (Odonata - Aeshnidae). Revista de la Sociedad Entomológica Argentina, 26, 71-73. Retrieved from https://www.biotaxa.org/RSEA/article/view/43160

Belle, J. (1964). Surinam dragonflies of the genus Aphylla, with a description of a new species. Studies on the Fauna of Suriname and other Guyanas, 7(23), 22-34.

Belle, J. (1970). Studies on South American Gomphidae (Odonata) with special reference to the species from Surinam. Studies on the Fauna of Suriname and other Guyanas, 11(43), 1-158.

Belle, J. (1988). A synopsis of the species of Phyllocycla Calvert with descriptions of four new taxa and a key to the genera of Neotropical Gomphidae (Odonata, Gomphidae). Tijdschrift Voor Entomologie, 131, 73-102.

Belle, J. (1992). Studies on ultimate instar larvae of neotropical Gomphidae, with the description of Tibiagomphus gen. nov. (Anisoptera). Odonatologica, 21(1), 1-24. Retrieved from http://natuurtijdschriften.nl/record/591970

Belle, J. (2002). Commented checklist of the Odonata of Surinam. Odonatologica, 31(1), 1-8. Retrieved from http://natuurtijdschriften.nl/record/592378

Bick, G. H. (1953). The nymph of Miathyria marcella (Selys) (Odonata, Libellulidae). Proceedings of the Entomological Society of Washington, 55(1), 30-36.

Borges, L. R., Barbosa, M. S., Vilela, D., \& Carneiro, M. A. A. (2019). Dragonflies and damselflies (Insecta: Odonata) from a Cerrado area at Triângulo Mineiro, Minas Gerais, Brazil. Biota Neotropica, [online], 19(1), e20180609. doi:10.1590/1676-0611-bn-2018-0609

Borror, D. J. (1942). A revision of Libelluline genus Erythrodiplax (Odonata). Ohio State University Graduate Studies, Contributions in Zoology and Entomology 4, Biological Series xv $+286 \mathrm{pp}$.

Bota-Sierra, C.A. (2014). Eight new records for Colombia from the Orinoco Basin (Odonata: Calopterygidae, Coenagrionidea, Lestdae, Heteragrionidea, Libellulidae). Notulae Odonatologicae, 8(4), 77-116.

Brown, A. D., \& Pacheco, S. (2006). Propuesta de actualización del mapa ecorregional de la argentina. In A. Brown, U. Martinez Ortiz, M. Acerbi, \& J. Corcuera (Eds.), La Situación Ambiental Argentina 2005 (pp. 28-31). Buenos Aires: Fundación Vida Silvestre Argentina.

Bulla, L. A. (1970). Contribución al conocimiento de los Zygoptera Argentinos. I. Las ninfas de Telebasis willinki Fraser y Argentagrion ambiguum (Ris) (Insecta, Odonata). Revista de la Sociedad Entomologica Argentina, 32(1-4), 91-98.

Bulla, L. A. (1971). Consideraciones sobre el género Argentagrion Fraser, 1947 con la descripción de una nueva especie (Odonata, Coenagriidae). Revista de la Sociedad Entomológica Argentina, 33(1-4), 49-55.

Bulla, L. A. (1972). La ninfa de Protallagma titicacae (Calvert) (Odonata, Coenagriidae). Neotropica, 18(57), $129-133$.

Bulla, L. A. (1973a). Cinco ninfas nuevas o poco conocidas del género Oxyagrion Selys (Odonata, Caenag.). Revista del Museo de La Plata (Nueva Serie), Sección Zoología, 12, 11-25.

Bulla, L. A. (1973b). Revisión de dos especies Argentinas del género Cyanallagma Kennedy (Odonata, Coenagriidae). Revista de la Sociedad Entomológica Argentina, 34(1-2), 95-105.

Bulla, L. A. (1973-1974). Clave para la identificacion de los Odonata Zygoptera de la Rcpublica Argentina al sur del paralelo $30^{\circ} \mathrm{S}$ (Odonata). Revista de la Sociedad Entomológica Argentina, 34(3-4), 217-228.

Byers, C. F. (1941). Notes on the emergence and life history of the dragonfly Pantala flavescens. Proceedings of the Florida Academy of Science, 6, 14-25. Retrieved from www.jstor.org/stable/24313165

Cabot, L. (1890). Immature state of the Odonata. Memoirs of the Museum of Comparative Zoology, 17(1), 1-52.

Calvert, P. P. (1909). Contribution to a knowledge of the Odonata of the Neotropical region, exclusive of Mexico and Central America. Annals of the Carnegie Museum, 6(1), 73-280.

Calvert, P. P. (1928). Report on Odonata, including notes on some internal organs of the larvae. Collected by the Barbados-Antigua Expedition from the University of Iowa in 1918. University of Iowa Studies, 12(2), 1-44.

Calvert, P. P. (1934). The rates of growth, larval development and seasonal distribution of dragonflies of the genus Anax (Odonata: Aeshnidae). Proceedings of the American Philosophical Society, 73(1), 1-70.

Calvert, P. P. (1956). The neotropical species of the "sub-genus Aeshna" sensu Selysii 1883 (Odonata). Memoirs of the American Entomological Society, 15, 1-251.

Campos, R. E. (1994). Importancia de las larvas de culícidos en la dieta de Ischnura fluviatilis (Odonata: Zygoptera) en habitats naturales de los alrededores de La Plata, provincia de Buenos Aires. Revista de la Sociedad Entomológica Argentina, 53(1-4), 51-56.

Carle, F. L., Kjer, K. M., \& May, M. L. (2015). A molecular phylogeny and classification of Anisoptera (Odonata). Arthropod Systematics \& Phylogeny, 73(2), 281-301.

Carle, F. L., \& Louton, J. A. (1994). The larva of Neopetalia punctata and establishment of Austropetaliidae fam. nov. (Odonata). Proceedings of the Entomological Society of Washington, 96(1), 147-155.

Carvalho, A. L. (1987). Description of the larva of Gynacantha bifida Rambur (Anisoptera: Aeshnidae). Odonatologica, 16(3), 281-284. Retrieved from http://natuurtijdschriften.nl/record/591792

Carvalho, A. L. (1988). Descrição da larva de Triacanthagyna ditzleri Williamson, 1923 (Odonata, Aeshnidae, Gynacanthini). Revista brasiliera de Entomologia, 32(2), 223-226. 
Carvalho, A. L. (1992). Aspectos da biologia de Coryphaeschna perrensi (McLachlan, 1887) (Odonata, Aeshnidae), com enfase no periodo larval. Revista brasiliera de Entomologia, 36(4), 791-802.

Carvalho, A. L. (1993). A morfologia externa da larva de ultimo estadio de Coryphaeschna perrensi (McLachlan, 1887) (Odonata, Aeshnidae). Revista brasiliera de Entomologia, 37(1), 167-179.

Carvalho, A. L., \& Ferreira, N. (1989). Descrição da larva de Gynacantha mexicana e notas sobre sua Biologia. Revista brasiliera de Entomologia, 33(3/4), 413-419.

Carvalho, A. L., Ferreira, N., \& Nessiminian, J. L. (1991). Descrição das larvas de três espécies do gênero Erythrodiplax Brauer (Odonata: Libellulidae). Revista brasileira de Entomologia, 35(1), 165-171.

Carvalho, A. L., \& Werneck-de-Carvalho, P. C. (2005). Descrição da larva de Orthemis cultriformis Calvert, 1899 (Insecta, Odonata, Libellulidae). Arquivos do Museu Nacional, Rio de Janeiro, 63(2), 267-273.

Carvalho, A. L., Werneck-de-Carvalho, P. C., \& Calil, E. R. (2002). Description of the larvae of two species of Dasythemis Karsch, with a key to the genera of Libellulidae occurring in the states of Rio de Janeiro and São Paulo, Brazil (Anisoptera). Odonatologica, 31(1), 23-33. Retrieved from http://natuurtijdschriften.nl/record/592380

Clausnitzer, V., Kalkman, V. J., Ram, M., Collen, B., Baillie, J. E. M., Bedjanic, M., Darwall, W. R.T., Dijkstra, K.-D. B., Dow, R., Hawking, J., Karube, H., Malikova, E., Paulson, D., Schütte, K., Suhling, F., Villanueva, R. J., von Ellenrieder, N., \& Wilson, K. (2009). Odonata enter the biodiversity crisis debate: The first global assessment of an insect group. Biological Conservation, 142(8), 1864-1869. doi:10.1016/j.biocon.2009.03.028

Costa, J. M. (1986). Contribução ao conhecimento da fauna da Guanabara. 59 - Notas sobre a ninfa de Epigomphus paludosus Hagen in Selys, 1854 (Odonata, Gomphidae). Atas da Sociedade de Biologia de Rio de Janeiro, 11(4), $157-158$

Costa, J. M., \& Carneiro, S. M. V. (1994). Duas novas larvas de Lestes Leach e dados morfológicos sobre Lestes pictus Hagen (Odonata, Zygoptera, Lestidae). Revista Brasileira de Zoología, 11(2), 303-309. doi:10.1590/S010181751994000200015

Costa, J. M., Carriço, C., Santos, T. C., \& Mascarenhas, B. J. A. (2010). Description of the final instar of Macrothemis heteronycha (Calvert) (Anisoptera: Libellulidae). Zootaxa, 2506, 65-68. doi:10.11646/zootaxa.2506.1.6

Costa, J. M., \& de Assis, C. V. (1994). Description of the larva of Tauriphila argo Hagen, 1869 from São Paulo, Brazil (Anisoptera: Libellulidae). Odonatologica, 23(1), 51-54. Retrieved from http://natuurtijdschriften.n1/record/592069

Costa, J. M., Machado, A. B. M., Lencioni, F. F. A., \& Santos, C. T. (2000). Diversidade e distribuição dos Odonata (Insecta) no Estado de São Paulo, Brasil: Parte I - Lista das espécies e registros bibliográficos. Publicações Avulsas do Museu Nacional, 80, 1-27.

Costa, J. M., Oldrini, B. B., \& Anjos Santos, D. (2009). Eight new Leptagrion larvae from Brazil (Zygoptera, Coenagrionidae). Odonatologica, 38(2), 93-111. Retrieved from http://natuurtijdschriften.nl/record/592655

Costa, J. M., \& Pujol-Luz, J. R. (1993). Descrição da larva de Erythemis mithroides (Brauer) e notas sobre outras larvas conhecidas do gênero (Odonata, Libellulidae). Revista brasileira de Zoologia, 10(3), 443-448. doi:10.1590/S0101-81751993000300011

Costa, J. M., Ravanello, C. T., \& Souza-Franco, G. M. (2008). The larva of Argia croceipennis Selys (Zygoptera: Coenagrionidae). Odonatologica, 37(3), 265-271. Retrieved from http://natuurtijdschriften.nl/record/592635

Costa, J. M., \& Régis, L. P. R. B. (2005). Description of the last larval instar of Perihemis thais (Perty) and comparison with other species of the genus (Anisoptera: Libellulidae). Odonatologica, 34(1), 51-57. Retrieved from http://natuurtijdschriften.nl/record/592499

Costa, J. M., \& Santos, T. C. (2000). Two new species of Santosia Costa, and Santos, 1992, with a description of five new corduliid larvae (Anisoptera: Corduliidae). Odonatologica, 29(2), 95-111. Retrieved from http://natuurtijdschriften.nl/record/592311

Costa, J. M., Souza, L. O. I., \& Santos, T. C. (2000). Two new species of Oxyagrion Selys, 1876, with a description of five new larvae (Zygoptera: Coenagrionidae). Odonatologica, 29(1), 1-15. Retrieved from http://natuurtijdschriften.n1/record/592302

Costa, J. M., Souza-Franco, G. M., \& Takeda, A. M. (1999). Descrição da larva de Diastatops intensa Montgomery, 1940 e morfologia dos diferentes estádios de desenvolvimiento (Odonata: Libellulidae). Boletim do Museo Nacional, N.S., 410, 1-14.

Costa, J. M., Vieira, L. P., \& Lourenço, A. N. (2001). Descrição de três larvas de Erythrodiplax Brauer, 1868, e redescrição das larvas de E. pallida (Needham, 1904) e E. umbrata (Linnaeus, 1758), com chave para identificação das larvas conhecidas das espécies Brasileiras (Odonata, Libellulidae). Boletim do Museo Nacional, N.S., $465,1-16$.

Dalzochio, M. S. (2009a). Descrição da larva de último estádio de Micrathyria pseudeximia Westfall (Odonata, Libellulidae). EntomoBrasilis, 2(2), 54-57. doi:10.12741/ebrasilis.v2i2.56

Dalzochio, M. S. (2009b). Descrição da larva de último ínstar de Nephepeltia berlai Santos, 1950 (Odonata, Libellulidae). EntomoBrasilis, 2(3), 70-72. doi:10.12741/ebrasilis.v2i3.60

Dalzochio, M. S., Périco, E., Renner, S., \& Sahlén, G. (2018). Description of the final stadium larva of Erythrodiplax media (Odonata: Libellulidae) with preliminary key to known South American larvae in the genus. International Journal of Odonatology, 21(2), 93-104. doi:10.1080/13887890.2018.1462260

Dalzochio, M. S., \& Rodrigues, M. E. (2011). Description of the larva of Archilestes exoletus (Hagen in Selys) (Odonata: Lestidae). Zootaxa, 2756, 65-68. doi:10.11646/zootaxa.2756.1.5

de Assis, C. V., \& Costa, J. M. (1994). Seis novas larvas do gênero Micrathyria Kirby e notas sobre a distribuição no Brasil (Odonata: Libellulidae). Revista Brasileira de Zoología, 11(2), 195-209. doi:10.1590/S010181751994000200003 
del Palacio, A., Diez, F., \& Latini, Y. (2017). Odonata from La Pampa province, Argentina. Odonatologica, 46(1/2), 25-34. doi:10.5281/zenodo.572354.

del Palacio, A., Lozano, F., \& Muzón, J. (2018). Description of the final instar larva of Argia serva Hagen in Selys, 1865 (Odonata Coenagrionidae). Anais da Academia Brasileira de Ciências, 90(3), 3017-3022. doi:10.1590/ 0001-3765201820180093.

del Palacio, A., \& Muzón, J. (2014). Description of the final instar larva of Limnetron antarcticum Förster and notes on its female (Anisoptera: Aeshnidae). Zootaxa, 3884(1), 89-94. doi:10.11646/zootaxa.3884.1.8

del Palacio, A., \& Muzón, J. (2016). Redescription of Erythrodiplax pallida (Needham, 1904) (Odonata: Libellulidae). International Journal of Odonatology, 19(1-2), 23-30. doi:10.1080/13887890.2016.1143408.

del Palacio, A., \& Muzón, J. (2019). Redescription of Erythrodiplax chromoptera Borror 1942 with comments on the morphology of the vesica spermalis (Odonata: Libellulidae). Zoologischer Anzeiger, 278, 90-94. doi:10.1016/j.jcz.2018.12.004

De Marmels, J. (1982a). Dos náyades nuevas de la familia Aeshnidae (Odonata: Anisoptera). Boletín de Entomología de Venezuela, Nueva Serie, 2(12), 102-106.

De Marmels, J. (1982b). Cuatro náyades nuevas de la familia Libellulidae (Odonata: Anisoptera). Boletin de Entomología de Venezuela, Nueva Serie, 2(11), 94-101.

De Marmels, J. (1990). Nine new Anisoptera larvae from Venezuela (Gomphidae, Aeshnidae, Corduliidae, Libelulidae). Odonatologica, 19(1), 1-15. Retrieved from http://natuurtijdschriften.nl/record/591890

De Marmels, J. (1992a). Odonata del Cerro Guaiquinima (Edo. Bolivar) y zonas aledañas. Boletín de Entomología Venezolana, 7(1), 37-47.

De Marmels, J. (1992b). Caballitos del Diablo (Odonata) de las Sierras de Tapirapecó y Unturán, en el extremo sur de Venezuela. Acta Biológica Venezuélica, 14(1), 57-78.

De Marmels, J. (2007). Thirteen new Zygoptera larvae from Venezuela (Calopterygidae, Polythoridae, Pseudostigmatidae, Platystictidae Protoneuridae, Coenagriondiae). Odonatologica, 36(1), 27-51. Retrieved from http://natuurtijdschriften.nl/record/592574

Dijkstra, K.-D. B., Bechly, G., Bybee, S. M., Dow, R. A., Dumont, H. J., Fleck, G., Garrison, R. W., Hämäläinen, M., Kalkman, V. J., Karube, H., May, M. L., Orr, A. G., Paulson, D. R., Rehn, A. C., Theischinger, G.Trueman, J. W. H., Van Tol, J., von Ellenrieder, N., \& Ware, J. (2013). The classification and diversity of dragonflies and damselflies (Odonata). Zootaxa, 3703(1), 36-45. doi:10.11646/zootaxa.3703.1.9

Fleck, G. (2003). Contribution à la connaissance des Odonates de Guyane française: Notes sur des larves des genres Orthemis, Diastatops et Elga (Anisoptera: Libellulidae). Odonatologica, 32(4), 335-344. Retrieved from http://natuurtijdschriften.nl/record/592448:

Fleck, G. (2004). Contribution à la connaissance des Odonates de Guyane française: les larves des Macrothemis pumilla Karsch, 1889 et de Brechmorhoga praedatrix Calvert, 1909. Notes biologiques et conséquences taxonomiques (Anisoptera: Libellulidae). Annales de la Société entomologique de France, 40(2), 177-184. doi:10.1080/ 00379271.2004 .10697414

Fleck, G., Brenk, M., \& Misof, B. (2006). DNA taxonomy and the identification of immature insect stages: the true larva of Tauriphila argo (Hagen 1869) (Odonata: Anisoptera: Libellulidae). Annales de la Société entomologique de France, 42(1), 91-98. doi:10.1080/00379271.2006.10697453

Fleck, G., De Marmels, J., \& Grand, D. (2004). La larve de Tholymis citrina Hagen, 1867 (Odonata, Anisoptera, Libellulidae). Bulletin de la Société entomologique de France, 109(5), 455-457. Retrieved from www.persee.fr/ doc/bsef_0037-928x_2004_num_109_5_16154

Förster, F. (1914). Beitrage zu den Gattungen und Arten der Libellen. III. Archiv für Naturgeschichte, 80, 59-83. doi:10.5962/bhl.part.26477

Fraser, F. C. (1947). The Odonata of the Argentine Republic I. Acta Zoológica Lilloana, 4, 427-461.

Fraser, F. C. (1948). The Odonata of the Argentine Republic II. Acta Zoológica Lilloana, 5, 47-67.

Garré, A., \& Lozano, F. (2007). Descripción del último estadio larval de Micrathyria ungulata (Odonata: Libellulidae). Revista de la Sociedad Entomológica Argentina, 66(1-2), 5-9.

Garré, A., Muzón, J., \& Ardohain, D. M. (2008). Description of the final instar larvae of Erythrodiplax atroterminata Ris and E. corallina (Brauer) (Odonata: Libellulidae). Zootaxa, 1896, 45-50. doi:10.5281/zenodo.184442

Garrison, R. W. (2006). A synopsis of the genera Mnesarete Cowley, Bryoplathanon gen. nov., and Ormenoplebia [sic Ormenophlebia] gen. nov. (Odonata: Calopterygidae). Contributions to Science Series, 506, 1-84.

Garrison, R. W. (2009). A synopsis of the genus Telebasis (Odonata: Coenagrionidae), International Journal of Odonatology, 12(1), 1-121. doi:10.1080/13887890.2009.9748331

Garrison, R. W., von Ellenrieder, N., \& Louton, J. A. (2006). The dragonfly genera (Odonata: Anisoptera) of the New World. An illustrated and annotated key. The Johns Hopkins University Press, Baltimore, 384 pp.

Geijskes, D. C. (1934). Notes on the Odonata fauna of the Dutch West Indian Islands Aruba, Curacao and Bonaire with an account on their nymphs. Internationale Revue der Gesamten Hydrobiologie und Hydrographie, 31, $284-311$. doi:10.1002/iroh.19340310116

Geijskes, D. C. (1941). Notes on Odonata of Surinam. II. Six mostly new zygopterous nymphs from coastland waters. Annals of the Entomological Society of America, 35(4), 719-134. doi:10.1093/aesa/34.4.719

Geijskes, D. C. (1946). Observations on the Odonata of Tobago, B.W.I. Transactions of the Royal Entomological Society of London, 97(9), 213-235. doi:10.1111/j.1365-2311.1946.tb00281.x

Geijskes, D. C. (1959). The aeschnine genus Staurophlebia. Studies on the Fauna of Suriname and other Guyanas, 3(9), 147-172. Retrieved from http://www.repository.naturalis.nl/record/506286 
Geijskes, D. C. (1968). Anax longipes versus Anax concolor. Notes on Odonata of Suriname X. Studies on the Fauna of Suriname and other Guyanas, 10, 67-100. Retrieved from http://www.repository.naturalis.nl/record/506293

Geijskes, D. C. (1986). The larva of Dicterias cothurnata (Förster, 1906) (Zygoptera: Dicteriastidae). Odonatologica, 15(1), 77-80. Retrieved from http://natuurtijdschriften.nl/record/591734

Guillermo-Ferreira, R., \& Bispo, P. C. (2012). Description of the larva of Mnesarete pudica (Hagen in Selys, 1853) (Odonata: Calopterygidae) and notes on known genera of South American Calopterygidae larvae. Zootaxa, 3482, 77-81. doi:10.11646/zootaxa.3482.1.5

Guillermo-Ferreira, R., \& Bispo, P. C. (2013). Description of the larva of Telebasis griffinii (Martin, 1896) (Zygoptera: Coenagrionidae). Odonatologica, 42(4), 383-387.

Hoffmann, J. (2009). Summary catalogue of the Odonata of Peru - Kommentiertes Faksimile des Manuskripts von J. COWLEY, Cambridge, 20.05.1933 und aktuelle Liste der Odonaten Perus mit Fundortangaben sowie Historie zu Sammlern und Odonatologen in Peru. IDF-Report, 16, 1-115.

Jurzitza, G. (1981). Lista provicional de los Odonatos del Parque Nacional Iguazú, provincia de Misiones República Argentina. Notulae Odonatologica, 1(7), 117-118. Retrieved from http://natuurtijdschriften.nl/record/593255

Kennedy, C. H. (1923). The naiad of Pantala hymenea. Canadian Entomologist, 54, 36-38. doi:10.4039/Ent5536-2

Klots, E. B. (1932). Insects of Porto Rico and the Virgin Islands, Odonata or dragonflies. Scientific Survey of Porto Rico and the Virgin Islands. New York Academy of Sciences, 16, 1-107.

Koroiva, R., Neiss, U. G., Fleck, G., \& Hamada, N. (2020). Checklist of dragonflies and damselflies (Insecta: Odonata) of the Amazonas state, Brazil. Biota Neotropica, [online], 20(1), e20190877. doi:10.1590/1676-0611-bn-2019-0877

Lencioni, F. A. A. (2004). Telagrion nathaliae spec. nov. (Zygoptera: Coenagrionidae). Odonatologica, 33(1), 91-98. Retrieved from http://natuurtijdschriften.nl/record/592462

Lencioni, F. A. A. (2017). Damselflies of Brazil. An illustrated identification guide. Southeast region. e-book. 1st edition.

Limongi, J. (1983). Estudio morfo-taxonómico de náyades en algunas especies de Odonata (Insecta) en Venezuela. Memorias de la Sociedad de ciencias naturales "La Salle", 43(119), 95-117.

Limongi, J. (1991). Estudio morfo-taxonómico de náyades de algunas especies de Odonata (Insecta) en Venezuela (II). Memorias de la Sociedad de ciencias naturales "La Salle", 49(131-132), 405-420.

Lozano, F. (2013). Description of three females of the genus Acanthagrion (Odonata: Coenagrionidae) with a key to the females of Argentina. Zootaxa, 3646(1), 23-38. doi:10.11646/zootaxa.3646.1.2

Lozano, F., Garré, A., \& Pessacq, P. (2007). Descripción del último estadio larval de Acanthagrion aepiolum (Odonata: Coenagrionidae). Revista de la Sociedad Entomológica Argentina, 66(1-2), 1-4.

Lozano, F., Muzón, J., \& del Palacio, A. (2011). Description of final stadium larva of Erythrodiplax connata and E. basifusca and redescription of that of E. minuscula (Odonata: Libellulidae). International Journal of Odonatology, 14(2), 127-135. doi:10.1080/13887890.2011.595354

Lozano, F., Muzón, J., \& Scattolini, C. (2012). Description of the final stadium larva of Telebasis obsoleta (Selys, 1876) (Odonata: Coenagrionidae). Zootaxa, 3186, 54-58. doi:10.11646/zootaxa.3186.1.4

Lozano, F., Muzón, J., \& Torres, S. (2009). Description of the final instar larva of Homeoura lindneri (Ris, 1928) and redescription of the final instar larva of $H$. chelifera (Selys, 1876) (Odonata: Coenagrionidae). Zootaxa, 2231, 47-54. doi:10.11646/zootaxa.2231.1.3

Lozano, F., Rodríguez, J. S., \& Molineri, C. (2017). Acanthagrion peruvianum Leonard, 1977 (Odonata Coenagrionidae) a junior subjective synonym of A. floridense Fraser, 1946 and description of its final stadium larva. Zoologischer Anzeiger, 270, 71-80. doi:10.1016/j.jcz.2017.10.002

Mauffray, W. F., \& Tennessen, K. J. (2019). A Catalogue and Historical Study of the Odonata of Ecuador. Zootaxa, 4628(1), 1-265. doi:10.11646/zootaxa.4628.1.1

Meurgey, F., \& Poiron, C. (2011). The true Dythemis multipunctata Kirby, 1894, from the West Indies and proposed new taxonomic status (Odonata: Anisoptera: Libellulidae). Zootaxa, 3019, 51-62. doi:10.11646/zootaxa.3019.1.3

Molineri, C., \& Rodríguez, J. S. (2013). Description of the larva of Argia jujuya Ris (Coenagrionidae) with a key to species from the Argentinean Yungas cloud forest. International Journal of Odonatology, 16(4), 301-307. doi:10.1080/13887890.2013.851628

Montgomery, B. E. (1940). A revision of the genus Diastatops (Libellulidae, Odonata) and a study of the leg characters of related genera. Lloydia, 3(4), 213-280. Retrieved from https://lib.dr.iastate.edu/rtd/13727

Muzón, J. (1993). Lestes spatula Fraser: description of the final larval instar and redescription of male and female adults (Zygoptera: Lestidae). Odonatologica, 22(4), 443-454. Retrieved from http://natuurtijdschriften.nl/record/592056

Muzón, J. (1997). Redescripción de Lestes auritus y Lestes paulistus y descripción del último estadio larval de L. undulatus (Odonata: Lestidae). Revista de la Sociedad Entomológica Argentina, 56(1-4), 159-166.

Muzón, J., del Palacio, A., \& Ramos, L. (2014). Ischnura ultima Ris, 1908 (Odonata: Coenagrionidae): New records from southern South America. CheckList, 10(1), 187-188. doi:10.15560/10.1.187

Muzón, J., \& Garré, A. (2005). Description of the last instar larva of Erythrodiplax paraguayensis (Förster) (Anisoptera: Libellulidae). Revista de la Sociedad Entomologica Argentina, 64(1-2), 85-91.

Muzón, J., \& Lozano, F. (2011). Description of the final instar larva of Progomphus joergenseni Ris (Epiprocta: Gomphidae). Zootaxa, 2762, 56-60. doi:10.11646/zootaxa.2762.1.5

Muzón, J., \& Lozano, F. (in press). Negragrion sagma gen.n. and sp.n. from South America with a morphological phylogeny of the New World Ischnurinae (Odonata: Zygoptera: Coenagrionidae). Anais da Academia Brasileira de Ciências.

Muzón, J., Lozano, F., del Palacio, A., Ramos, L. S., \& Lutz, A. (2015). Odonata from the Lower Delta of the Paraná River, Argentina. Agrion, 20(2), 68-72. 
Muzón, J., \& Pessacq, P. (2005). Description of the last larval instar of Ischnura ultima Ris (Zygoptera: Coenagrionidae). Odonatologica, 34(3), 303-306. Retrieved from http://natuurtijdschriften.nl/record/592524

Muzón, J., Pessacq, P., \& Lozano, F. (2014). The Odonata (Insecta) of Patagonia: A synopsis of their current status with illustrated keys for their identification. Zootaxa, 3784(4), 346-388. doi:10.11646/zootaxa.3784.4.2

Muzón, J., Pessacq, P., \& von Ellenrieder, N. (2006). Description of the female and larva of Phyllogomphoides joaquini Rodrigues Capitulo 1992 (Anisoptera, Gomphidae). Odonatologica, 35(1), 47-52. Retrieved from http://natuurtijdschriften.nl/record/592539

Muzón, J., Rodrigues Capitulo, A., \& Jurzitza, G. (1990). Populations dynamik von Telebasis willinki Fraser, 1948 im Galeriewald des Rio de la Plata bei Punta Lara, Argentinien (Odonata: Coenagrionidae). Opuscula Zoologica Fluminensia, 53, 1-10.

Muzón, J., \& von Ellenrieder, N. (1996). Estadios larvales de Odonata de la Patagonia. I. Descripción de Aeshna variegata Fabricius (Odonata: Aeshnidae). Revista de la Sociedad Entomológica Argentina, 56(1-4), 143-146.

Muzón, J., \& von Ellenrieder, N. (1997). Description of the last larval instar of Sympetrum villosum Ris (Odonata: Libellulidae). Neotropica, 43(109-110), 41-43.

Muzón, J., \& von Ellenrieder, N. (1998). Odonata. In J. J. Morrone \& S. Coscarón (Eds.), Biodiversidad de Artrópodos Argentinos. Una perspectiva biotaxonómica (pp. 14-25). La Plata, Argentina: Ediciones Sur.

Muzón, J., \& von Ellenrieder, N. (2001). Revision of the subgenus Marmaraeschna (Odonata: Aeshnidae). International Journal of Odonatology, 4(2), 93-124. doi:10.1080/13887890.2001.9748167

Muzón, J., von Ellenrieder, N., \& Pessacq, P. (2001). Description of the last larval instar of Acanthagrion hildegarda Gloger, 1967 (Odonata: Coenagrionidae). Revista de la Sociedad Entomológica Argentina, 60(1-4), 95-98.

Muzón, J., \& Weigel Muñoz, S. (2007). Description of the final instar larva of Lestes dichrostigma Calvert (Zygoptera: Lestidae). Studies on Neotropical Fauna and the Environment, 42(3), 235-239. doi:10.1080/01650520601106196

Muzón, J., Weigel Muñoz, S., \& Campos, R. (2009). Description of the bromeliad-dwelling final instar larva of Leptagrion andromache Hagen in Selys (Zygoptera: Coenagrionidae). Zootaxa, 2089, 65-68. doi:10.11646/zootaxa.2089.1.6

Muzón, J., Weigel Muñoz, S., \& Campos, R. (2010). Description of the last instar larva of Mecistogaster amalia (Burmeister) (Odonata: Pseudostigmatidae). International Journal of Odonatology, 13(1), 137-144. doi:10.1080/13887890.2010.9748367

Navás, L. (1917). Algunos insectos Neurópteros de la Argentina. Physis, 3, 186-196.

Navás, L. (1920). Insectos sudamericanos. Anales de la Sociedad Científica Argentina, 90, 44-51.

Navás, L. (1922). Insectos Sudamericanos. Cuarta Serie (1). Revista de la Real Academia de Ciencias Exactas Físicas y Naturales de Madrid, 19, 255-267.

Navás, L. (1924). Insectos de la Argentina y Chile. Estudios (Buenos Aires), 22, 358-368.

Navás, L. (1927a). Insectos nuevos de la República Argentina. Revista de la Sociedad Entomológica Argentina, 1, $27-29$.

Navás, L. (1927b). Insectos de la Argentina y Chile. Tercera Serie. Estudios (Buenos Aires), 33, 22-28.

Navás, L. (1928). Insectos de la Argentina. Cuarta Serie. Estudios (Buenos Aires), 35, 139-147.

Navás, L. (1930). Insectos de la Argentina. Sexta Serie. Revista de la Sociedad Entomológica Argentina, 3(2), $125-132$.

Needham, J. G. (1904). New dragonfly nymphs in the United States National Museum. Proceedings of the United States National Museum, 27, 685-720. doi:10.5479/si.00963801.27-1371.685

Needham, J. G. (1940). Studies on Neotropical Gomphine dragonflies (Odonata). Transactions of the American Entomological Society, 65, 363-394. Retrieved from www.jstor.org/stable/25077446

Needham, J. G. (1941). Life history studies on Progomphus and its nearest allies (Odonata: Aeschnidae). Transactions of the American Entomological Society, 67, 221-245. Retrieved from www.jstor.org/stable/25077477

Needham, J. G. (1944). Further studies on Neotropical Gomphinae (Odonata). Transactions of the American Entomological Society, 69, 171-224. Retrieved from www.jstor.org/stable/25077514

Needham, J. G., \& Bullock, D. S. (1943). The Odonata of Chile. Zoological Series of the Field Museum, 24(32), 357-373. doi:10.5962/bhl.title.3057

Needham, J. G., \& Westfall, M. J. (1955). A manual of the dragonflies of North America (Anisoptera). Berkeley: University of California Press, xii $+615 \mathrm{pp}$.

Needham, J. C., Westfall, M. J., \& May, M. L. (2000). Dragonflies of North America. (rev. ed.). Gainesville, FL: Scientific Publishers. $\mathrm{xv}+939 \mathrm{pp}$.

Neiss, U. G., Fiorentin, G. L., \& De Marmels, J. (2011). The larva of Allopodagrion brachyurum De Marmels, 2001 (Odonata: Zygoptera: Megapodagrionidae) from Southern Brazil. Zootaxa, 2836, 44-50. doi:10.11646/ zootaxa.2836.1.3

Novelo Gutiérrez, R. (2005). La larva de Enallagma novaehispaniae Calvert, 1902 (Odonata: Coenagrionidae). Folia Entomológica Mexicana, 44(2), 219-224.

Novelo Gutiérrez, R., \& Ramírez, A. (1998). The larva of Macrothemis inacuta (Odonata: Libellulidae). Entomological News, 109(5), 301-306.

Oldrini, B. B., \& Mascarenhas, B. J. de A. (2005). Descrição da larva de Idiataphe longipes (Odonata, Libellulidae, Trameini). Iheringia, Série Zoologica, 95(4), 431-433. doi:10.1590/S0073-47212005000400014

Perez Gutierrez, L. A., \& Palacino Rodriguez, F. (2011). Updated checklist of the Odonata known from Colombia. Odonatologica, 40(3), 203-225.

Pessacq, P. (2007). Peristica aenoviridis Calvert, 1909 and P. forceps Hagen in Selys, 1860: redescriptions and a new synonymy (Zygoptera: Protoneuridae). Odonatologica, 36(2), 207-218. Retrieved from http://natuurtijd schriften.nl/record/592589 
Pessacq, P. (2008). Protoneuridae. In L. E. Claps, G. Debandi, \& S. Roig-Juñet (Eds.), Biodiversidad de Artrópodos Argentinos Vol. II. (pp. 181-184). Mendoza: Editorial Sociedad Entomológica Argentina.

Pessacq, P. (2014). Synopsis of Epipleoneura (Zygoptera, Coenagrionidae, "Protoneuridae"), with emphasis on its Brazilian species. Zootaxa, 3872(3), 201-234. doi:10.11646/zootaxa.3872.3.1

Pessacq, P., \& Brand, C. (2009). Description of the larva of Phyllopetalia apollo Selys and redescription of that of Hypopetalia pestilens McLachlan (Anisoptera: Austropetaliidae). Odonatologica, 38(3), 235-246. Retrieved from http://natuurtijdschriften.nl/record/592666

Pessacq, P., Lozano, F., \& Muzón, J. (2015). A checklist of the dragonflies from the North-Western of Isiboro-Sécure Indian Country and National Park, Bolivia. Agrion, 20(2), 64-66.

Pessacq, P., \& Muzón, J. (2004). Description of the final stadium larva of Hetaerina rosea Selys (Zygoptera: Calopterygidae). Studies on Neotropical Fauna and Environment, 39(3), 239-242. doi:10.1080/01650520400007363

Pessacq, P., Muzón, J., \& von Ellenrieder, N. (2005). Description of the last larval instar of Acanthagrion ablutum Calvert (Zygoptera: Coenagrionidae). Odonatologica, 34(1), 73-76. Retrieved from http://natuurtijdschriften.nl/ record/592502

Pujol-Luz, J. R. (1990). Descrição da larva de Elasmothemis constricta (Calvert, 1898) (Odonata: Libellulidae). Revista brasileira de Biologia, 50(2), 487-490.

Rambur, P. (1842). Histoire Naturelle des Insectes: Neuropteres. Paris: Libraire Encyclopedique de Roret, xvii +534 pp.

Ramírez, A. (1995). Descripción e historia natural de las larvas de odonatos de Costa Rica. IV. Mecistogaster ornata (Rambur, 1842) Zygoptera: Pseudostigmatidae). Bulletin of American Odonatology, 3(2), 43-47.

Ramos, L. S., Lozano, F., \& Muzón, J. (2017). Odonata diversity and synanthropy in urban areas: a case study in Avellaneda City, Buenos Aires, Argentina. Neotropical Entomology, 46(2), 144-150. doi:10.1007/s13744-016-0443-5

Renner, S., Périco, E., Ely, G. J., \& Sahlén, G. (2017). Preliminary dragonfly (Odonata) species list from the Pampa biome in Rio Grande do Sul, Brazil, with ecological notes for 19 new records for the State Biota Neotropica, [online], 17(4), e20170374. doi:10.1590/1676-0611-bn-2017-0374

Ris, F. (1904). Odonaten. Hamburg Magallaenischen Sammelreise 1892/93. Hamburg: Friederichsen, and Co., $44 \mathrm{pp}$.

Ris, F. (1913). Neuer Beitrag zur Kenntnis der Odonatenfauna von Argentina. Memoires de la Societe Entomologique de Belgique, 22, 55-102.

Ris, F. (1918). Libellen (Odonaten) aus der Region der amerikanischen Kordilleren von Costarica bis Catamarca. Archiv für Naturgeschichte Abteilung A., 82(9), 1-197.

Ris, F. (1928). Die Ausbeute der Deutschen Chaco-Expedition 1925-26. Konowia, 7(1), 40-49.

Rodrigues Capitulo, A. (1980). Contribución al conocimiento de los Anisoptera de la República Argentina. I. Descripción de los estadios preimaginales de Aeshna bonariensis Rambur (Insecta Odonata). Limnobios, 2(1), 1-21.

Rodrigues Capitulo, A. (1981). Presencia de Anax amazili Burmeister (Odonata Anactinae) en la República Argentina. Algunos datos acerca del comportamiento y determinación del metabolismo energético de las ninfas. Limnobios, 2(4), 207-214

Rodrigues Capitulo, A. (1983a). La ninfa de Phyllocycla argentina (Hagen in Selys) 1878 (Odonata, Gomphidae). Revista de la Sociedad Entomológica Argentina, 42(1-4), 267-271.

Rodrigues Capitulo, A. (1983b). Descripción de los estadios preimaginales de Erythemis attala Selys (Odonata Libellulidae). Limnobios, 2(7), 533-548.

Rodrigues Capitulo, A. (1985). Una nueva especie del género Cyanogomphus Selys, incluyendo la descripción del último estadio preimaginal (Odonata Gomphidae). Revista de la Sociedad Entomológica Argentina, 43(1-4), 329-336.

Rodrigues Capitulo, A. (1992). Los Odonata de la República Argentina (Insecta). Fauna de Agua Dulce de la República Argentina, 34(1), 1-91.

Rodrigues Capitulo, A. (1996). Description of the last instar larva of Tauriphila risi Martin (Anisoptera: Libellulidae). Odonatologica, 25(4), 391-395. Retrieved from http://natuurtijdschriften.nl/record/592180

Rodrigues Capitulo, A. (2000). Population dynamics of larval stages of Tauriphila risi Martin and Erythemis attala (Selys) in Punta Lara gallery forest, BuenosAires, Argentina (Anisoptera: Libellulidae). Odonatologica, 29(4), 333340. Retrieved from http://natuurtijdschriften.nl/record/592333

Rodrigues Capitulo, A., \& Jurzitza, G. (1989). Erstbeschreibung der Larve von Castoraeschna decurvata Dunkle \& Cook 1984 (Odonata: Aeshnidae). Entomologische Zeitschrift, 99(21), 312-317.

Rodrigues Capitulo, A., Mola, L., \& Agopian, S. (1991). Species catalogue and chromosomal data of Odonata from Argentina. Revista de la Sociedad Entomológica Argentina, 48(1-4), 59-72.

Rodrigues Capitulo, A., \& Muzón, J. (1985). Anisopteros del Parque Nacional "El Palmar”, prov. Entre Ríos (Odonata). Revista de la Sociedad Entomológica Argentina, 44(2), 128.

Rodrigues Capitulo, A., \& Muzón, J. (1990). The larval instars of Orthemis nodiplaga Karsch 1891 from Argentina (Anisoptera: Libellulidae). Odonatologica, 19(3), 283-291. Retrieved from http://natuurtijdschriften.nl/record/ 591915

Rodrigues da Fonseca, R., \& Pujol-Luz, J. R. (1999). Notas sobre a larva de Ischnura fluviatilis Selys, 1876 (Odonata, Coenagrionidae). Contribuições Avulsas sobre a História Natural do Brasil, Série Zoología, 1, 1-4.

Rodríguez, J., Gómez, D., \& Molineri, C. (2014). Nuevos registros de Odonata y Ephemeroptera para el noroeste de Argentina. Revista de la Sociedad Entomológica Argentina, 73(1-2), 85-88.

Rodríguez, J., Gómez, D., \& Molineri, C. (2018). New records of Odonata from Argentina. Odonatologica, 47(1/2), 193-212. doi:10.5281/zenodo. 1481092 
Rodríguez, J., \& Molineri, C. (2013). Diversidad del orden Odonata (Fabricius, 1793) en la provincia de Tucumán, Argentina. Acta Zoológica Lilloana, 57(1), 22-30.

Rodríguez, J., \& Molineri, C. (2014). Description of the final instar larva of Rhionaeschna vigintipunctata (Ris, 1918) (Odonata: Aeshnidae). Zootaxa, 3884(3), 267-274. doi:10.11646/zootaxa.3884.3.5

Salgado, L. G. V., Carvalho, A. L., \& Pinto, A. P. (2013). Larval taxonomy of Macrothemis Hagen, 1868 (Odonata: Libellulidae), with descriptions of four larvae and a key to the fourteen known species. Zootaxa, 3599(3), $229-245$. doi:10.11646/zootaxa.3599.3.2

Santos, N. D. (1953). Fauna do Distrito Federal. II. Redescrição de "Erythrodiplax anomala" (Brauer, 1865) Brauer, 1868. (Odonata; Libellulidae). Anais da Academia Brasileira de Ciências, 25, 499-504.

Santos, N. D. (1967). Notas sôbre a ninfa de Erythrodiplax connata fusca (Rambur, 1842) Brauer, 1868 (Odonata, Libellulidae). Atas da Sociedade de Biologia do Rio de Janeiro, 10(6), 145-147.

Santos, N. D. (1968a). Contribuição ao conhecimento da fauna do estado da Guanabara. 61 - Notas sobre a ninfa de Progomphus complicatus (?) Selys 1854 e seu imago (Gomphidae, Odonata). Atas da Sociedade de Biologia do Rio de Janeiro, 11(5), 171-174.

Santos, N. D. (1968b). Contribuição ao conhecimento da fauna do estado da Guanabara. 62 - Notas sobre a ninfa e o imago de Micrathyria hypodidyma Calvert, 1906. Atas da Sociedade de Biologia do Rio de Janeiro, 11(5), $195-197$.

Santos, N. D. (1968c). Contribuição ao conhecimento da fauna do estado da Guanabara. 66 - Descrição da ninfa de Trapezostigma cophysa (Selys, 1857) Cowley, 1934 e notas sobre a emergencia (Odonata, Libellulidae). Atas da Sociedade de Biologia do Rio de Janeiro, 12(3), 169-171.

Santos, N. D. (1968d). Fauna do estado da Guanabara. 65. Descrição da ninfa de Heteragrion aurantiacum Selys, 1862 e notas sobre o imago (Odonata, Megapodagrionidae). Atas da Sociedade de Biologia do Rio de Janeiro, 12(1), $13-15$.

Santos, N. D. (1969a). Notas sobre a ninfa e o imago de Coryphaeschna perrensi (MacLachlan, 1887) Ris, 1913 (Odonata, Aeshnidae). Atas da Sociedade de Biologia do Rio de Janeiro, 12(4), 173-174.

Santos, N. D. (1969b). Contribuição ao conhecimento da fauna do estado da Guanabara. 67 - Descrição da ninfa e emergencia de Brechmorhoga nubecula (?) (Rambur, 1842) Calvert, 1898 (Odonata - Libellulidae). Atas da Sociedade de Biologia do Rio de Janeiro, 12(4), 221-223.

Santos, N. D. (1969c). Contribuição ao conhecimento da fauna do estado da Guanabara. 69 - Descrição da ninfa de Erythemis credula (Hagen, 1861) Calvert 1907 (Odonata: Libellulidae). Atas da Sociedade de Biologia do Rio de Janeiro, 12(5, 6), 287-288.

Santos, N. D. (1970a). Contribuição ao conhecimento da fauna do estado da Guanabara. 73 - Notas sobre a ninfa, o imago e a emergencia de Coryphaeschna adnexa (Hagen, 1861) Calvert, 1903 (Odonata, Aeshnidae). Atas da Sociedade de Biologia do Rio de Janeiro, 13(1, 2), 75-77.

Santos, N. D. (1970b). Descrição da ninfa de Macrothemis musiva (Hagen, 1861) Calvert, 1898 (Odonata: Libellulidae). Atas da Sociedade de Biologia do Rio de Janeiro, 13(5, 6), 157-158.

Santos, N. D. (1972a). Contribuição ao conhecimento da fauna do estado da Guanabara. 79 - Descrição da ninfa de Lestes pictus Selys, 1862 (Odonata: Lestidae). Atas da Sociedade de Biologia do Rio de Janeiro, 15(2), 77-78.

Santos, N. D. (1972b). Contribuição ao conhecimento da fauna do estado da Guanabara e arredores. 80 - Descrição da ninfa de Micrathyria artemis (Selys ms.) Ris, 1911 (Odonata: Libellulidae). Atas da Sociedade de Biologia do Rio de Janeiro, 15(3), 141-143.

Santos, N. D. (1973a). Contribuição ao conhecimento da fauna da Guanabara e arredores 82 - Descrição da ninfa de Gynacantha gracilis (Burmeister, 1839) Kolbe, 1888 (Aeshnidae: Odonata). Atas da Sociedade de Biologia do Rio de Janeiro, 16(2, 3), 55-57.

Santos, N. D. (1973b). Contribuição ao conhecimento da fauna da Guanabara e arredores. 81 - Descrição da ninfa de Triacanthagyna caribbea Williamson, 1923 (Odonata: Aeshnidae). Atas da Sociedade de Biologia do Rio de Janeiro, $16(2,3), 53-54$.

Santos, N. D. (1973c). Contribuição ao conhecimento da fauna does estado da Guanabara e arredores. 84 - Descrição da ninfa de Perithemis mooma Kirby, 1889 (Odonata: Libellulidae). Atas da Sociedade de Biologia do Rio de Janeiro, $16(2,3), 71-72$.

Santos, N. D. (1978). Contribuição ao conhecimento da fauna do Municipio do Rio de Janeiro, RJ e arredores 85 Descrição da ninfa de Micrathyria atra (Martin, 1897) Calvert, 1906 (Odonata: Libellulidae). Atas da Sociedade de Biologia do Rio de Janeiro, 19, 17-18.

Santos, N. D., \& Costa, J. (1987). Descrição da ninfa de Chalcopteryx rutilans (Rambur, 1842) Selys, 1853 (Odonata: Polythoridae). Atas da Sociedade de Biologia do Rio de Janeiro, 8(27), 30-34.

Santos, N. D., \& Costa, J. (1988). The larva of Heliocharis amazona Selys, 1853 (Zygoptera: Heliocharitidae). Odonatologica, 17(2), 135-139. Retrieved from http://natuurtijdschriften.n1/record/591822

Santos, N. D., Costa, J. M., \& Pujol-Luz, J. R. (1993). Descrição da larva de Diastatops obscura (Fabricius) (Odonata, Libellulidae). Revista Brasileira de Zoología, 10(3), 467-472. doi:10.1590/S0101-81751993000300014

Schmidt, E. (1941). Petaluridae, Gomphidae und Petaliidae der Schonemannschen Sammlung aus Chile (Ordnung Odonata). Archiv für Naturgeschichte, N.F., 10(2), 231-258.

Schröder, N. M., Anjos-Santos, D., Rippel, C. G., \& Pessacq, P. (2020). Description of the last instar larva of Peristicta aeneoviridis Calvert, 1909 (Odonata: Coenagrionidae). Zootaxa, 4728(4), 461-468. doi:10.11646/zootaxa.4728.4.4

Souza, L. O. I., \& Costa, J. M. (2002). Descrição de tres larvas de Micrathyria Kirby, 1889, com chave para identificação das larvas conhecidas das espécies Brasileiras (Odonata, Libellulidae). Arquivos do Museu Nacional, Rio de Janeiro, 60(4), 321-331. 
Souza, L. O. I., Costa, J. M., \& Espindola, L. A. (2002). Description of the last instar larva of Oligoclada laetitia Ris, 1911 and comparison with other Libellulidae (Anisoptera). Odonatologica, 31(4), 403-407. Retrieved from http://natuurtijdschriften.nl/record/592416

Souza, L. O. I., Costa, J. M., \& Santos, T. C. (1999). Redescrição da larva de Tramea calverti Muttkowski, 1910, com chave para identificação das larvas conhecidas do gênero (Odonata, Libellulidae). Boletim do Museu Nacional, Rio de Janeiro, N.S., 409, 1-7.

Souza, L. O. I., Pepinelli, M., \& Neiss, U. G. (2012). The larva of Neoneura ethela Williamson, 1917 (Odonata: Protoneuridae). Zootaxa, 3318, 63-67. doi:10.11646/zootaxa.3318.1.5

Spindola, L. A., Souza, L. O. I., \& Costa, J. M. (2001). Descrição da larva de Perithemis thais Kirby, 1889, com chave para identificação das larvas conhecidas do gênero citadas para o Brasil (Odonata, Libellulidae). Boletim do Museu Nacional, Rio de Janeiro, N.S., 442, 1-8.

Tennessen, K. J. (2009). Aeolagrion philipi sp. nov. from Bolivia, and a review of the genus Aeolagrion (Odonata: Coenagrionidae). International Journal of Odonatology, 12(2), 309-322. doi:10.1080/13887890.2009.9748347

Theischinger, G., \& Watson. J. A. L. (1984). Larvae of Australian Gomphomacromiinae, and their bearing on the status of the Synthemis group of genera (Odonata: Corduliidae). Australian Journal of Zoology, 32, 67-95. doi:10.1071/ZO9840067

Vivas-Santeliz, J., \& De Marmels, J. (2017). Current knowledge of Odonata in Venezuela: diversity and distribution of endemic taxa. Odonatologica, 46(1/2), 35-54. doi:10.5281/zenodo.572355

von Ellenrieder, N. (1999). Description of the last larval instar of Aeshna (Hesperaeschna) cornigera planaltica Calvert, 1952 (Odonata: Aeshnidae). Revista de la Sociedad Entomológica Argentina, 58(3-4), 151-156.

von Ellenrieder, N. (2001a). The larvae of Patagonian Aeshna Fabricius species (Anisoptera: Aeshnidae). Odonatologica, 30(4), 423-434. Retrieved from http://natuurtijdschriften.nl/record/592372

von Ellenrieder, N. (2001b). A synopsis of the Patagonian species of the genus Aeshna Fabricius (Anisoptera: Aeshnidae). Odonatologica, 30(3), 299-325. Retrieved from http://natuurtijdschriften.nl/record/592362

von Ellenrieder, N. (2001c). Species composition and distribution patterns of the Argentinian Aeshnidae (Odonata: Anisoptera). Revista de la Sociedad Entomológica Argentina, 60(1-4), 39-60.

von Ellenrieder, N. (2005). Taxonomy of the South American genus Phyllopetalia (Odonata: Austropetaliidae). International Journal of Odonatology, 8(2), 311-352. doi:10.1080/13887890.2005.9748258

von Ellenrieder, N. (2006). The larvae of Teinopodagrion decipiens De Marmels, 2001 and T. meridionale De Marmels, 2001 (Zygoptera: Megapodagrionidae). Odonatologica, 35(3), 281-287. Retrieved from http://natuurtijdschriften.nl/record/592560

von Ellenrieder, N. (2007a). The larva of Hetaerina mendezi Jurzitza, with comments on H. rosea Selys (Zygoptera: Calopterygidae). Odonatologica, 36(4), 405-414. Retrieved from http://natuurtijdschriften.nl/record/592608

von Ellenrieder, N. (2007b). The larva of Argia joergenseni Ris, 1913 (Zygoptera: Coenagrionidae). Odonatologica, 36(1), 89-94. Retrieved from http://natuurtijdschriften.nl/record/592580

von Ellenrieder, N. (2007c). Some Libellulidae larvae from the Yungas Forest, Argentina: Macrothemis hahneli Ris, Brechmorhoga nubecula (Rambur) and Dasythemis mincki clara Ris (Anisoptera). Odonatologica, 36, 263-273. Retrieved from http://natuurtijdschriften.nl/record/592593

von Ellenrieder, N. (2008). Revalidation of Argentagrion and redefinition of Homeoura, with the description of H. obrieni n. sp. (Odonata: Coenagrionidae). Revista de la Sociedad Enomológica Argentina, 67(1-2), 81-106.

von Ellenrieder, N. (2009). Andinagrion garrisoni. The IUCN Red List of Threatened Species 2009: e.T158729A5274111. Retrieved August 20, 2019, from http://dx.doi.org/10.2305/IUCN.UK.2009-2.RLTS.T158729 A5274111.en

von Ellenrieder, N. (2009). Progomphus costalis. The IUCN Red List of Threatened Species 2009: e.T159115A5316531. Retrieved July 18, 2019, from http://dx.doi.org/10.2305/IUCN.UK.2009-2.RLTS.T159115A5316531.en

von Ellenrieder, N. (2010). Odonata biodiversity of the Argentine Chaco biome. International Journal of Odonatology, 13(1), 1-25. doi:10.1080/13887890.2010.9748357

von Ellenrieder, N. (2011). Chapter 3. Odonata (dragonflies and damselflies) of the Kwamalasamutu region, Suriname. In B. J. O'Shea, L. E. Alonso, \& T. H. Larsen (Eds.), A rapid biological assessment of the Kwamalasamutu region, south-western Suriname (pp. 56-78). RAP Bulletin of Biological Assessment 63.

von Ellenrieder, N. (2012). The levis group of Orthemis revisited: a synopsis including a synonymy and description of six new species of Orthemis from South America (Odonata: Libellulidae). International Journal of Odonatology, 15(3), 115-207. doi:10.1080/13887890.2012.688186

von Ellenrieder, N. (2013). A revision of Metaleptobasis Calvert (Odonata: Coenagrionidae) with seven synonymies and the description of eighteen new species from South America. Zootaxa, 3738, 1-155. doi:10.11646/zootaxa.3738.1.1

von Ellenrieder, N. (2014). A synopsis of the Neotropical genus Nephepeltia (Odonata: Libellulidae), including description of a new species, synonymies, and a key to males. Zootaxa, 3796, 121-146. doi:10.11646/zootaxa.3796.1.6

von Ellenrieder, N., Billink, B., \& Svensson, E. I. (2017). Checklist of the dragonflies and damselflies from Guyana (Insecta: Odonata), with new records from the country. Check List, 13(2), 1-22. doi:10.15560/13.2.2104

von Ellenrieder, N., \& Garrison, R. W. (2003). A synopsis of the genus Triacanthagyna (Odonata: Aeshnidae). International Journal of Odonatology, 6(2), 147-184. doi:10.1080/13887890.2003.9748385

von Ellenrieder, N., \& Garrison, R. W. (2006). Rediscovery of Oxyagrion bruchi Navás, 1924 from Argentina, with a description of its larva (Zygoptera: Coenagrionidae). Pan-Pacific Entomologist, 82(3/4), 362-374.

von Ellenrieder, N., \& Garrison, R. W. (2007). Dragonflies and Damselflies (Insecta: Odonata) of the Argentine Yungas: Species composition and identification. Scientific Reports. 7. Società Zoologica "La Torbiera”, Italy, 105 pp. _ doubt 
von Ellenrieder, N., \& Garrison, R. W. (2008a). The genus Oligoclada in Argentina, with description of $O$. rubribasalis sp. nov. (Odonata: Libellulidae). International Journal of Odonatology, 11(2), 249-260. doi:10.1080/ 13887890.2008.9748327

von Ellenrieder, N., \& Garrison, R. W. (2008b). A redefinition of Telagrion Selys and Aceratobasis Kennedy stat. rev. and the description of Schistolobos gen. nov. for Telagrion boliviense Daigle (Odonata: Coenagrionidae). Transactions of the American Entomological Society, 134(1 + 2), 1-22. Retrieved from www.jstor.org/stable/25078986

von Ellenrieder, N., \& Lozano, F. (2008). Blues for the red Oxyagrion: a redefinition of the genera Acanthagrion and Oxyagrion (Odonata: Coenagrionidae). International Journal of Odonatology, 11(1), 95-113. doi:10.1080/13887890.2008.9748315

von Ellenrieder, N., Molineri, C., \& Emmerich, D. (2009). Odonata de Uruguay: lista de especies y nuevos registros. Revista de la Sociedad Entomológica Argentina, 68(1-2), 227-230.

von Ellenrieder, N., \& Muzón, J. (1999). The Argentinean species of the genus Perithemis Hagen (Anisoptera: Libellulidae). Odonatologica, 28(4), 385-398. Retrieved from http://natuurtijdschriften.nl/record/592294

von Ellenrieder, N., \& Muzón, J. (2000). Description of the last larval instar of Erythrodiplax nigricans (Rambur) (Anisoptera: Libellulidae). Odonatologica, 29(3), 267-272. Retrieved from http://natuurtijdschriften.n1/record/ 592326

von Ellenrieder, N., \& Muzón, J. (2003a). Description of the last larval instar of Aeshna (Marmaraeschna) pallipes Fraser, 1947 (Anisoptera: Aeshnidae). Odonatologica, 32(1), 95-98. Retrieved from http://natuurtijdschriften.nl/ record/592429

von Ellenrieder, N., \& Muzón, J. (2003b). Description of the last larval instar of Ischnura fluviatilis Selys (Zygoptera: Coenagrionidae). Bulletin of American Odonatology, 7(3), 57-60.

von Ellenrieder, N., \& Muzón, J. (2006). The genus Andinagrion (Zygoptera: Coenagrionidae), with description of a new species, Andinagrion garrisoni, and its larva from Argentina. International Journal of Odonatology, 9(2), $205-223$. doi:10.1080/13887890.2006.9748279

von Ellenrieder, N., \& Muzón, J. (2008). An updated checklist of the Odonata from Argentina. Odonatologica, 37(1), 55-68. Retrieved from http://natuurtijdschriften.nl/record/592615

Wasscher, M. T., \& Van 'T Bosch, J. G. (2013). The true identity of Neoneura bilinearis Selys, 1860, with the synonymy of N. gaida Rácenis, 1953, and the description of N. confundens sp. nov. (Odonata: Protoneuridae). Zootaxa, 3599, 19-36. doi:10.11646/zootaxa.3599.1.2

Weigel Muñoz, S., Ramos, L., \& Muzón, J. (2019). La Reserva Costera Municipal de Avellaneda (Buenos Aires, Argentina), una nueva área protegida dedicada al estudio de los odonatos. Hetaerina, 1(1), 12-13,

Westfall, Jr M. J. (1953). The nymph of Miathyria marcella Selys (Odonata). Florida Entomologist, 36, 21-25. Retrieved from www.jstor.org/stable/3492178

Westfall, Jr M. J. (1988). Elasmothemis gen. nov., a new genus related to Dythemis (Anisoptera: Libellulidae). Odonatologica, 17(4), 419-428. Retrieved from http://natuurtijdschriften.n1/record/591847

Zapata, A. I., \& Pereyra, M. C. (2016). Odonatos asociados al curso superior y medio del río Suquía, Córdoba, Argentina. Revista de la Sociedad Entomológica Argentina, 75(3-4), 135-138. 